\title{
INTERPLANETARY TRANSFER TRAJECTORIES USING THE INVARIANT MANIFOLDS OF HALO ORBITS
}

\author{
A Thesis \\ presented to \\ the Faculty of California Polytechnic State University, \\ San Luis Obispo
}

\author{
In Partial Fulfillment \\ of the Requirements for the Degree \\ Master of Science in Aerospace Engineering
}

by

Megan S. Rund

June 2018 
(C) 2018

Megan S. Rund

ALL RIGHTS RESERVED 


\section{COMMITTEE MEMBERSHIP}

TITLE:

Interplanetary Transfer Trajectories Using

the Invariant Manifolds of Halo Orbits

AUTHOR:

Megan S. Rund

DATE SUBMITTED: June 2018

COMMITTEE CHAIR: Kira Jorgensen Abercromby, Ph.D.

Associate Professor of Aerospace Engineering

COMMITTEE MEMBER: Jordi Puig-Suari, Ph.D.

Professor of Aerospace Engineering

COMMITTEE MEMBER: Eric Mehiel, Ph.D.

Professor of Aerospace Engineering

COMMITTEE MEMBER: Paige Parsons

Professional Aerospace Engineer 


\begin{abstract}
Interplanetary Transfer Trajectories Using the Invariant Manifolds of Halo Orbits
\end{abstract}

Megan S. Rund

Throughout the history of interplanetary space travel, the Newtonian dynamics of the two-body problem have been used to design orbital trajectories to traverse the solar system. That is, that a spacecraft orbits only one large celestial body at a time. These dynamics have produced impressive interplanetary trajectories utilizing numerous gravity assists, such as those of Voyager, Cassini, Rosetta and countless others. But these missions required large amounts of $\Delta v$ for their maneuvers and therefore large amounts of fuel mass. As we desire to travel farther and more extensively in space, these two-body dynamics lead to impossibly high $\Delta v$ values, and missions become infeasible due to the massive amounts of fuel that they would need to carry. In the last few decades a new dynamical system has been researched in order to find new ways of designing mission trajectories: the N-body problem. This utilizes the gravitational acceleration from multiple celestial bodies on a spacecraft, and can lead to unconventional, but very useful trajectories.

The goal of this thesis is to use the dynamics of the Circular Restricted ThreeBody Problem (CRTBP) to design interplanetary transfer trajectories. This method of modelling orbital dynamics takes into account the gravitational acceleration of two celestial bodies acting on a spacecraft, rather than just one. The invariant manifolds of halo orbits about Sun-planet Lagrange points are used to aid in the transfer from one planet to another, and can lead into orbital insertion about the destination planet or flyby trajectories to get to another planet. This work uses this method of dynamics to test transfers from Earth to both Jupiter and Saturn, and compares $\Delta v$ and time of flight values to traditional transfer methods. Using the CRTBP can lead to reduced $\Delta v$ amounts for completing the same missions as two-body dynamics would. The 
aim of this work is to research if using manifolds for interplanetary transfers could be superior for some high $\Delta v$ missions, as it could drastically reduce the required $\Delta v$ for maneuvers. With this method it could be possible to visit more distant destinations, or carry more mass of scientific payloads, due to the reduced fuel requirements.

Results of this research showed that using manifolds to aid in interplanetary transfers can reduce the $\Delta v$ of both departure from Earth and arrival at a destination planet. For transfers to Jupiter the $\Delta v$ for the interplanetary transfer was reduced by $4.12 \mathrm{~km} / \mathrm{s}$ compared to starting and ending in orbits about the planets. For a transfer to Saturn the $\Delta v$ required for the interplanetary transfer was reduced by $6.77 \mathrm{~km} / \mathrm{s}$. These $\Delta v$ savings are significant and show that utilizing manifolds can lead to lower energy interplanetary transfer trajectories, and have the potential to be useful for high $\Delta v$ missions. 


\section{ACKNOWLEDGMENTS}

I would like to thank my wonderful parents, and sister for always being there to support me and my craziness, and help me make it through this degree. I also would like to thank my amazing advisor, Dr. Kira Abercromby, for all of her help and patience, and for inspiring me to want to learn all about orbital mechanics. 


\section{TABLE OF CONTENTS}

$\begin{aligned} \text { Page } & \end{aligned}$

LIST OF TABLES . . . . . . . . . . . . . . . . . $\quad$ ix

LIST OF FIGURES . . . . . . . . . . . . . . . . . . . . . CHAPTER

1 Introduction . . . . . . . . . . . . . . . . . . 1

1.1 Statement of Problem . . . . . . . . . . . . . . . . . . 1

1.2 Proposed Solution . . . . . . . . . . . . . . . . 1

1.3 Past Research and Missions . . . . . . . . . . . . . . . 3

2 The Circular Restricted Three Body Problem . . . . . . . . . . . . . . 6

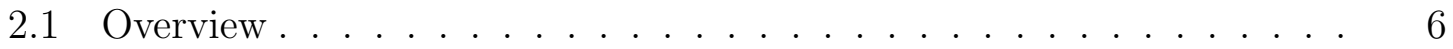

2.2 Synodic Coordinate Frame and Equations of Motion . . . . . . . . . 7

2.3 Lagrange Points . . . . . . . . . . . . . . . . . . 8

2.4 Libration Orbits . . . . . . . . . . . . . . . . . . . . . . . . . . . . . 10

2.5 Manifolds . . . . . . . . . . . . . . . . . . . . . 12

3 Halo Orbits and Invariant Manifolds . . . . . . . . . . . . . . . . 17

3.1 Overview . . . . . . . . . . . . . . . . 17

3.2 Halo Orbit Analytical Solution . . . . . . . . . . . . . . . . 20

3.3 Halo Orbit Numerical Solution . . . . . . . . . . . . . . . 26

3.4 Invariant Manifolds . . . . . . . . . . . . . . . . . . . 30

4 Maneuvers for Interplanetary Transfers . . . . . . . . . . . . . . . . . . 33

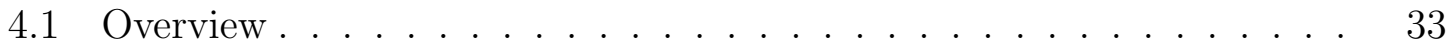

4.2 Earth to Halo Transfer . . . . . . . . . . . . . . . . . . . 33

4.3 Interplanetary Transfer . . . . . . . . . . . . . . . . . 38

4.3.1 Patched Conics with Manifolds Method . . . . . . . . . . . 39

4.3.2 Manifold to Hyperbolic Escape Trajectory Method . . . . . . 40

5 Mission Applications and Results . . . . . . . . . . . . . . . . . 49

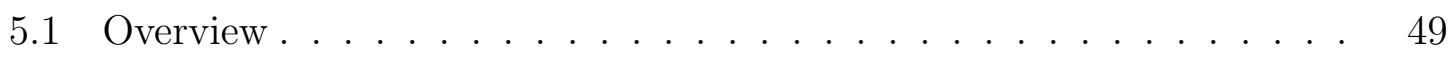

5.2 Earth to Jupiter Transfer . . . . . . . . . . . . . . . . . . . . . 49

5.3 Earth to Saturn Transfer . . . . . . . . . . . . . . . 57 
6 Conclusion . . . . . . . . . . . . . . . . . . . 65

6.1 Conclusions ....................... 65

6.2 Future Work . . . . . . . . . . . . . . . 66

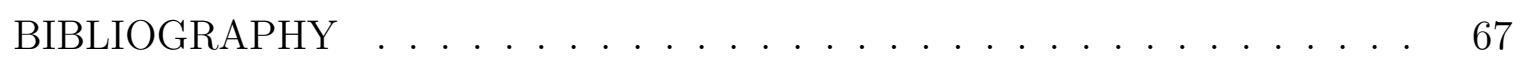
APPENDICES

A Orbital Elements Calculation . . . . . . . . . . . 69

B Velocity Vector Calculation ............... 71 


\section{LIST OF TABLES}

Table

4.1 $\Delta v$ and parking orbit altitudes for minimized parking orbit altitude and minimized $\Delta v$ for various halo orbit sizes. . . . . . . . . . 36

4.2 Comparison of total $\Delta v$ to get to halo orbit using higher and lower altitude parking orbits for a halo orbit with $\mathrm{A}_{\mathrm{z}}$ equal to $500,000 \mathrm{~km}$.

$5.1 \Delta v$ values for manifold-hyperbolic transfer for various sized halos at Earth and Jupiter. . . . . . . . . . . . . . . . . . . 51

5.2 Comparison of the $\Delta v$ required for traditional patched conics maneuvers and for the manifold maneuvers. . . . . . . . . . . . . 56

5.3 Comparison of the total $\Delta v$ required for mission and the time of flight (ToF) for traditional and manifold methods. . . . . . . . . . 57

$5.4 \Delta v$ values for manifold-hyperbolic transfer for various sized halos at Earth and Saturn. . . . . . . . . . . . . . . . . 58

5.5 Comparison of the $\Delta v$ required for traditional patched conics maneuvers and for the manifold maneuvers. . . . . . . . . . . . .

5.6 Comparison of the total $\Delta v$ required for mission and the time of flight $(\mathrm{ToF})$ for traditional and manifold methods. . . . . . . . . 


\section{LIST OF FIGURES}

Figure

1.1 Trajectory of Genesis Mission, showing the approach trajectory (purple), halo orbit (red), and return trajectory (blue). . . . . . . . . . 3

1.2 Artist rendition of interplanetary superhighway concept developed at JPL [9] . . . . . . . . . . . . . . . . . . . . . . . .

1.3 The trajectory of the WMAP mission to get to $\mathrm{L}_{2}$ using a trajectory within the stable manifold and lunar flybys[14]. . . . . . . . . . 5

2.1 The Synodic Coordinate Frame used for the CRTBP. . . . . . . . . 7

2.2 The five Lagrange points for the Earth-Moon system. . . . . . . . . 10

2.3 Example of Lissajous orbit about Sun-Earth $\mathrm{L}_{1}[6]$. . . . . . . . . . 11

2.4 Example of a halo orbit about Sun-Earth $\mathrm{L}_{1}$. . . . . . . . . . 11

2.5 The stable manifolds approaching Earth-Moon $\mathrm{L}_{2}$. . . . . . . . . . 12

2.6 The unstable manifolds departing Earth-Moon $\mathrm{L}_{2}$. . . . . . . . . . 13

2.7 The unstable invariant manifold for a halo orbit about Sun-Jupiter $\mathrm{L}_{2}$; z-amplitude of $300,000 \mathrm{~km}$. . . . . . . . . . . . . 15

$3.1 \quad$ Halo orbit about Sun-Earth $\mathrm{L}_{1}$; z-amplitude of 110,000 km. . . . . . 18

3.2 Example of a northern class halo (above) and southern class halo (below) about Sun-Earth $\mathrm{L}_{1}$; z-amplitude of 110,000 km. . . . . . . 18

3.3 Family of northern class halo orbits about Sun-Earth $\mathrm{L}_{1}$; z-amplitude of 110,000 to $1,100,000 \mathrm{~km}$. . . . . . . . . . . . . . . . . 19

3.4 Analytically estimated halo orbit compared with the actual trajectory when propagated with the equations of motion. . . . . . . .

3.5 Comparison of the halo orbit trajectories generated by the numerical and analytical solutions. . . . . . . . . . . . . 30

3.6 Example the stable invariant manifold for Sun-Jupiter $L_{2}$. . . . . . 32

4.1 An example of the 200 trajectories within the stable invariant manifold that pass closest to Earth for a halo about $\mathrm{L}_{2}$. . . . . . . . . .

4.2 Example of transfer trajectory from Earth parking orbit to halo with $\mathrm{A}_{\mathrm{z}}$ equal to $300,000 \mathrm{~km}$. . . . . . . . . . . . . . . . 38

4.3 Close up view of transfer point from Earth parking orbit onto trajectory within the stable invariant manifold. . . . . . . . . . . 
4.4 Hyperbolic escape trajectory geometry. . . . . . . . . . . . . . . . . 42

4.5 Hyperbolic arrival trajectory geometry. . . . . . . . . . . . . . . 43

4.6 Example of intersection point between the trajectory within the unstable invariant manifold (purple) and the hyperbolic escape trajectory (green) to leave Earth. . . . . . . . . . . . . . . . . . . 46

4.7 Earth departure example showing full manifold trajectory. . . . . . 47

4.8 Example of transfer point from incoming hyperbolic trajectory onto a trajectory within a manifold at Jupiter. . . . . . . . . . . . . . . 47

4.9 Jupiter arrival example showing full manifold trajectory. . . . . . . 48

5.1 Transfer trajectory about the sun from Earth to Jupiter, showing Earth's position at departure and Jupiter's position at arrival. . . .

5.2 Full view of transfer from halo orbit (blue) to trajectory within manifold (purple) to hyperbolic escape trajectory (green). . . . . . . . .

5.3 Close up views of transfer from manifold (purple) onto hyperbolic escape trajectory (green) at Earth. The yellow star shows the departure point on the manifold and the red star shows the injection point on the hyperbola. . . . . . . . . . . . . . . . .

5.4 Full view of transfer from hyperbolic escape trajectory (green) to trajectory within manifold (purple) to halo orbit (blue). . . . . . .

5.5 Close up views of transfer from hyperbolic escape trajectory (green) to manifold (purple) upon arrival at Jupiter. The yellow star shows the injection point on the manifold and the red star shows the departure point on the hyperbola. . . . . . . . . . . . . . . . .

5.6 Transfer from Earth to Saturn about the sun, showing Earth at the time of departure and Saturn at the time of arrival. . . . . . . . .

5.7 Full view of transfer from halo orbit (blue) to trajectory within manifold (purple) to hyperbolic escape trajectory (green). . . . . . . .

5.8 Close up views of transfer from manifold (purple) onto hyperbolic escape trajectory (green) at Earth. The yellow star shows the departure point on the manifold and the red star shows the injection point on the hyperbola. . . . . . . . . . . . . . .

5.9 Full view of transfer from hyperbolic escape trajectory (green) to trajectory within manifold (purple) to halo orbit (blue). . . . . .

5.10 Close up views of transfer from hyperbolic escape trajectory (green) to manifold (purple) upon arrival at Saturn. The yellow star shows the injection point on the manifold and the red star shows the departure point on the hyperbola. . . . . . . . . . . . . . . . 
Chapter 1

\section{INTRODUCTION}

\subsection{Statement of Problem}

Throughout most of the history of space travel, orbital dynamicists have used the two-body problem to model the motion of spacecraft. This method has led to the impressive trajectories of missions such as Voyager, Cassini, Juno and countless others. Although the orbital mechanics of these missions were optimized to reduce $\Delta v$ as much as possible, they still required large amounts of fuel to reach their destinations. As humans desire to explore farther and more extensively in space, the traditional two-body dynamics of past missions will start to yield too high $\Delta v$ values for feasible missions [7].

N-body dynamics have only been researched for use in missions for the last few decades, but could reduce the $\Delta v$ and therefore fuel required dramatically. By using these methods to design orbital trajectories, missions that would have been impossible could become feasible, and the extents of exploration through the solar system could reach new levels.

\subsection{Proposed Solution}

The Circular Restricted Three Body Problem (CRTBP) is a solution to the dynamics of a system with two primary bodies and one secondary body or spacecraft. The motion of these types of systems can create very unique and advantageous solutions to complicated trajectory problems. Finding trajectories using the solved equations

of motion for this three body system has the potential to dramatically reduce required 
$\Delta v$ for interplanetary transfer trajectories.

This thesis will test the idea of using a 3-body dynamical system rather than the traditional 2-body system to design interplanetary transfer trajectories from Earth. Then these trajectories can be connected to more transfers from planet to planet using CRTBP or can lead into flyby trajectories about another planet. Often the maneuvers that require the most $\Delta v$ for an interplanetary mission are the ones to escape Earth's sphere of influence and then enter into orbit about another planet. If 3-body dynamics can be used to reduce the $\Delta v$ required for these maneuvers, then missions can either carry less fuel and more payloads or visit more distant planets.

The method of creating these trajectories will utilize the invariant manifolds of halo orbits about Sun-planet Lagrange points. These manifolds are the most useful for creating interplanetary trajectories [7]. Manifolds can be used for low energy transfers from Low Earth Orbit (LEO) to a halo orbit about a Lagrange point and then on to a hyperbolic trajectory to escape the Earth's sphere of influence. Then, at a destination planet, the invariant manifolds of a halo orbit can be used to depart from the arrival hyperbolic trajectory and get onto a path staying in the Sun-planet system, or a flyby maneuver can be completed.

This method will be tested for transfers to Jupiter and Saturn, so that $\Delta v$ and time of flight values can be compared to transfers that only uses 2-body dynamics. While using manifolds can often increase the time of flight required, the reduced $\Delta v$ values could make these types of trajectories worthwhile for some interplanetary missions. As has been seen with the long mission lifetimes of spacecraft such as Voyager, large amounts of time in space can be accomplished. Utilizing the trajectories created by 3-body dynamics could then expand the reaches of where our spacecraft could travel. 


\subsection{Past Research and Missions}

Research on using the CRTBP for space mission trajectory design only really started in the 1960s. Since then, only a few missions have used these types of trajectories to accomplish their goals. Research has also been conducted on using these dynamics to design orbital trajectories travelling throughout the solar system and about the numerous moons of large planets such as Jupiter and Saturn.

Genesis was a mission designed by NASA's Jet Propulsion Laboratory (JPL) that travelled to a halo orbit about Sun-Earth $\mathrm{L}_{1}$ to collect solar wind samples. It then followed a trajectory to return to Earth and bring the samples back to be analyzed on the surface. Genesis utilized trajectories within invariant manifolds to get to a halo orbit about $\mathrm{L}_{1}$ and back to Earth using very little fuel, and was only possible due to the low energy transfers found using the CRTBP [12]. The trajectory that Genesis used can be seen in Figure 1.1, where the red trajectory is the halo orbit used to collect samples and the blue is the transfer back to Earth.

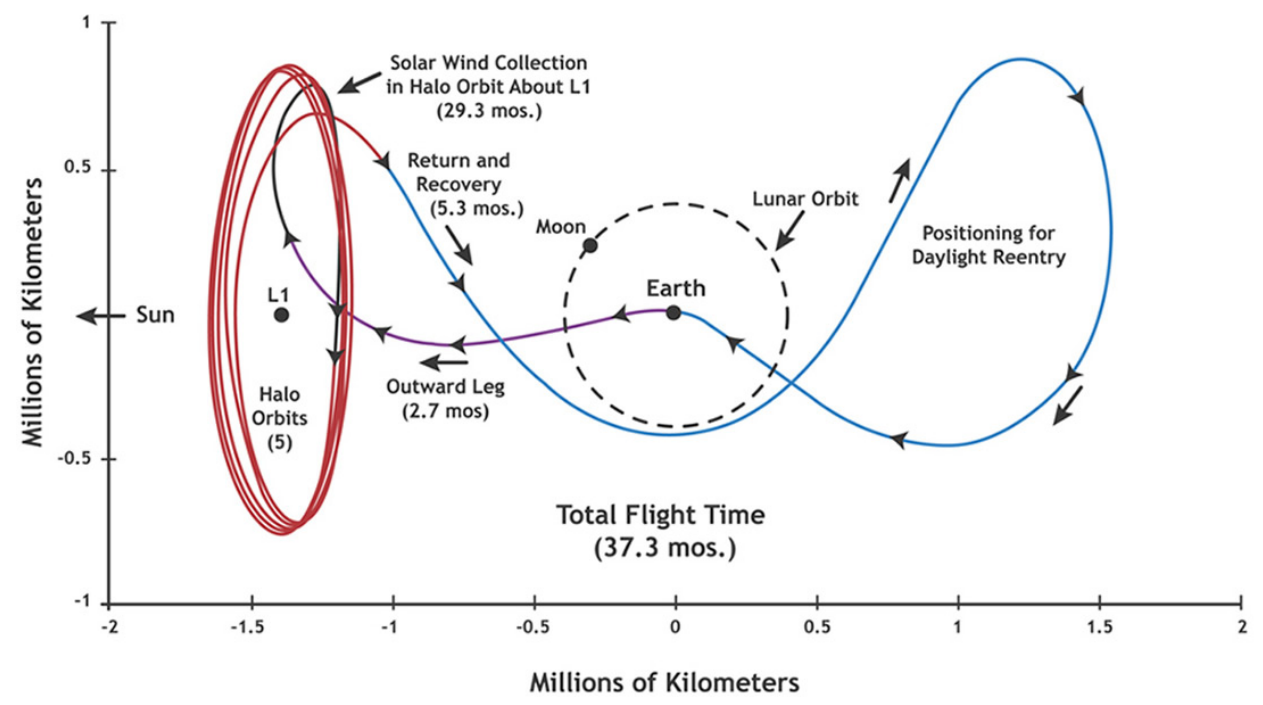

Figure 1.1: Trajectory of Genesis Mission, showing the approach trajectory (purple), halo orbit (red), and return trajectory (blue). 
Koon, Lo, Marsden and Ross have done significant research on using invariant manifolds for travel throughout the solar system. They have explored the possibilities of using these dynamics for interplanetary transfers as well as tours of systems of moons, such as those of Jupiter. They have also explored the idea of a lunar gateway station for interplanetary trajectories at Earth-Moon $\mathrm{L}_{1}$ [7]. At JPL, Martin Lo has also been part of developing the concept of the interplanetary superhighway that utilizes manifolds to travel throughout the solar system with very low energy transfers. An artist concept of this idea is shown in Figure 1.2.

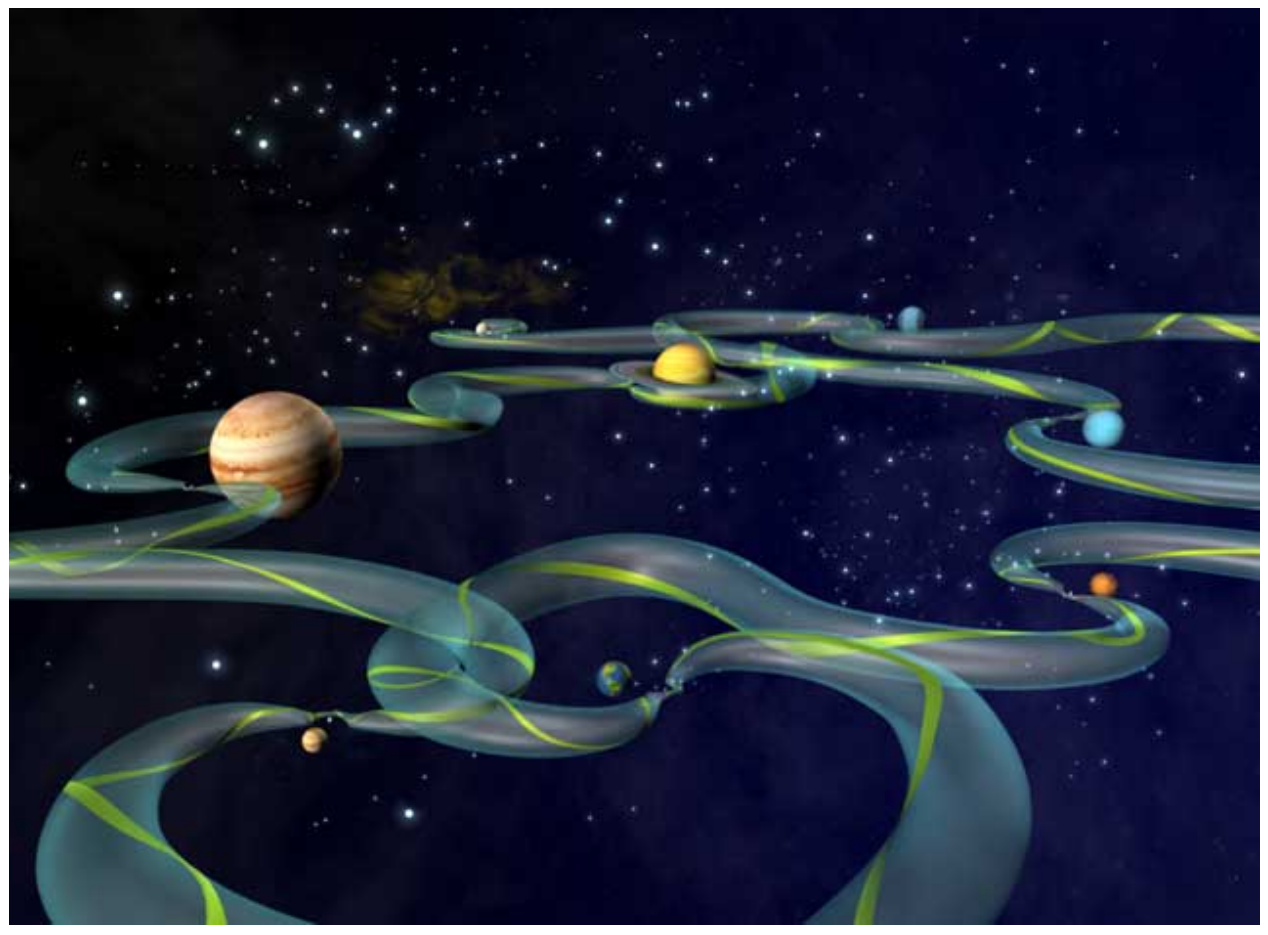

Figure 1.2: Artist rendition of interplanetary superhighway concept developed at JPL $[9]$.

Other missions that have utilized the dynamics of the CRTBP to complete their trajectory requirements are the Solar and Heliospheric Observatory (SOHO), WIND, Advanced Composition Explorer (ACE), WMAP, and ISEE-3 [7]. SOHO and ISEE-3 are joint missions between the European Space Agency (ESA) and NASA that also travelled to $L_{1}$ in order to observe the sun and gather data about its interior, surface, 
atmosphere, solar wind, and interactions with the Earth's magnetosphere [3],[10]. WIND and ACE are NASA missions that orbit about $\mathrm{L}_{1}$ in order to study solar wind and energetic particles emitted from the sun [13], [1]. WMAP is a NASA mission that travelled to $\mathrm{L}_{2}$ to measure the properties of cosmic microwave background radiation of space. This mission travelled to $\mathrm{L}_{2}$ using manifolds as well as multiple lunar flybys which can be seen in Figure 1.3 [14].

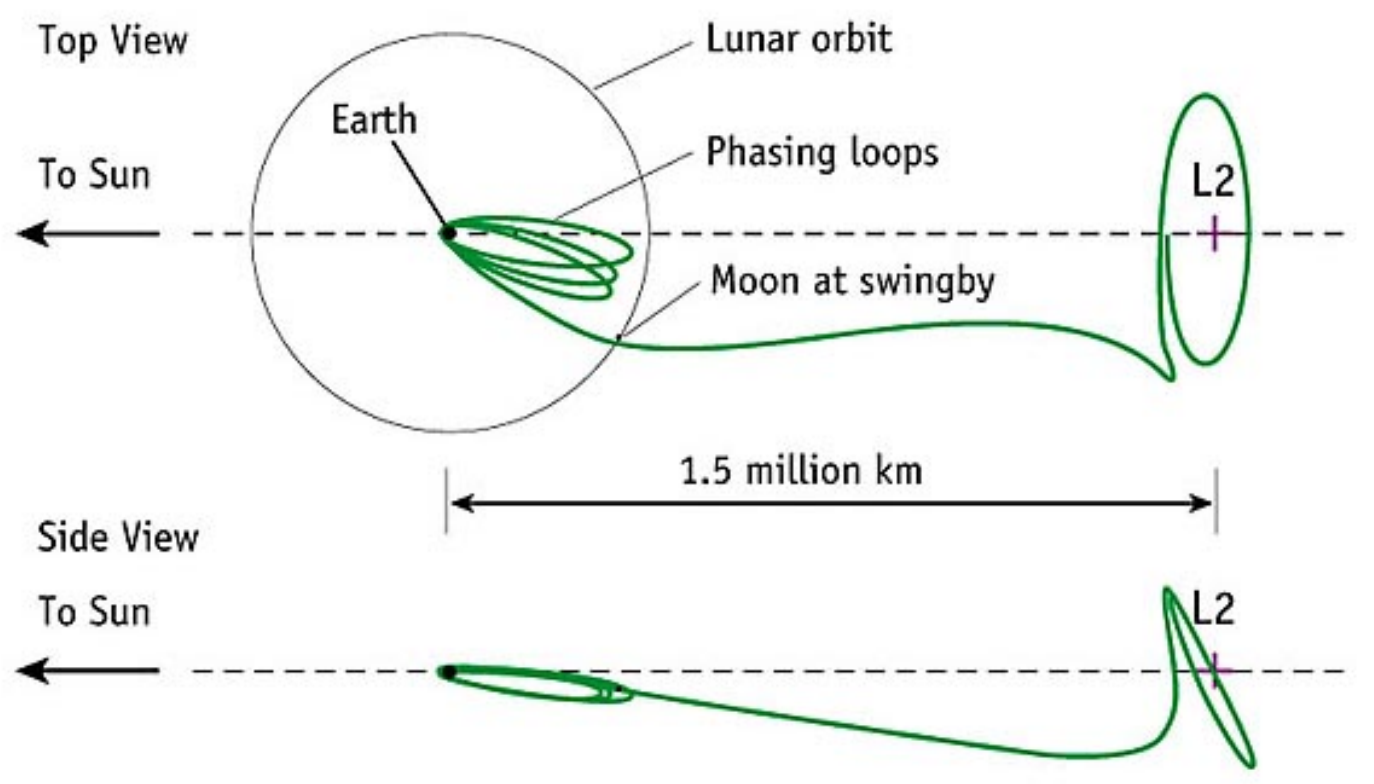

Figure 1.3: The trajectory of the WMAP mission to get to $\mathrm{L}_{2}$ using a trajectory within the stable manifold and lunar flybys[14].

The most current mission that is planning on using the dynamics of the CRTBP is the James Webb Space Telescope run by NASA, ESA, and the Canadian Space Agency (CSA), which is set to launch in 2020. It is a space-based infrared observatory that will orbit about $\mathrm{L}_{2}$ in order to look into the most distant and oldest galaxies. This mission uses the dynamics of the CRTBP in its final halo orbit and journey there in order to accomplish its objectives [11]. 
Chapter 2

\section{THE CIRCULAR RESTRICTED THREE BODY PROBLEM}

\section{$2.1 \quad$ Overview}

The majority of orbital trajectories are calculated using the two-body problem; that is with the satellite being the secondary body and the Sun, Earth, moon etc. being the primary body. Obviously perturbational effects from the gravitational pull of other large celestial bodies are often included to get a better understanding of how the actual orbit will behave, but the satellite is still in orbit about the one primary body. The Circular Restricted Three Body Problem (CRTBP) on the other hand, considers the effects of two large primary bodies' gravitational forces acting on one small secondary body. The small secondary body is assumed to have a mass that is small enough that its gravitational effects upon the system are negligible. The other important assumption in the CRTBP is that the orbits of the two primary bodies about the center of mass of the system are circular [17]. Without these assumptions the problem of solving the equations of the dynamics of the system becomes impossible. These assumptions are acceptable for most cases involving celestial bodies of the solar system, since most of the planets' orbits about the sun have very low eccentricities, and any spacecraft would have a mass many orders of magnitude smaller than a planet or moon.

The dynamics of the CRTBP can create very useful and interesting orbital trajectories that can be far more complex than typical elliptical and hyperbolic orbits usually encountered in the two-body problem. The next few sections will cover some of the unique dynamics of the CRTBP in more detail. 


\subsection{Synodic Coordinate Frame and Equations of Motion}

The synodic coordinate frame is the most commonly used reference frame for the CRTBP. The origin is defined at the center of mass of the two primary bodies of the system (the barycenter, B), and the frame rotates about the fixed barycentric coordinate frame with an angular velocity of $\omega_{\mathrm{s}}$. A diagram of the synodic coordinate frame is shown in Figure 2.1. In this graphic $\mathrm{m}_{1}$ is the more massive primary body, and $\mathrm{m}_{2}$ is the smaller of the two primaries. The distances $r_{\mathrm{B}, 1}$ and $\mathrm{r}_{\mathrm{B}, 2}$ are the distances from $\mathrm{m}_{1}$ and $\mathrm{m}_{2}$ to the barycenter of the system. The vectors $\overrightarrow{r_{1}}$ and $\overrightarrow{r_{2}}$ are the distances from the two primary bodies to the spacecraft and $\vec{r}_{B, s c}$ is the distance from the barycenter to the spacecraft. $\hat{x}_{\mathrm{s}}$ and $\hat{\mathrm{y}}_{\mathrm{s}}$ are the unit vectors representing the $\mathrm{x}$ and $\mathrm{y}$ axes of the synodic frame while the $\mathrm{z}$ axis points out of the page.

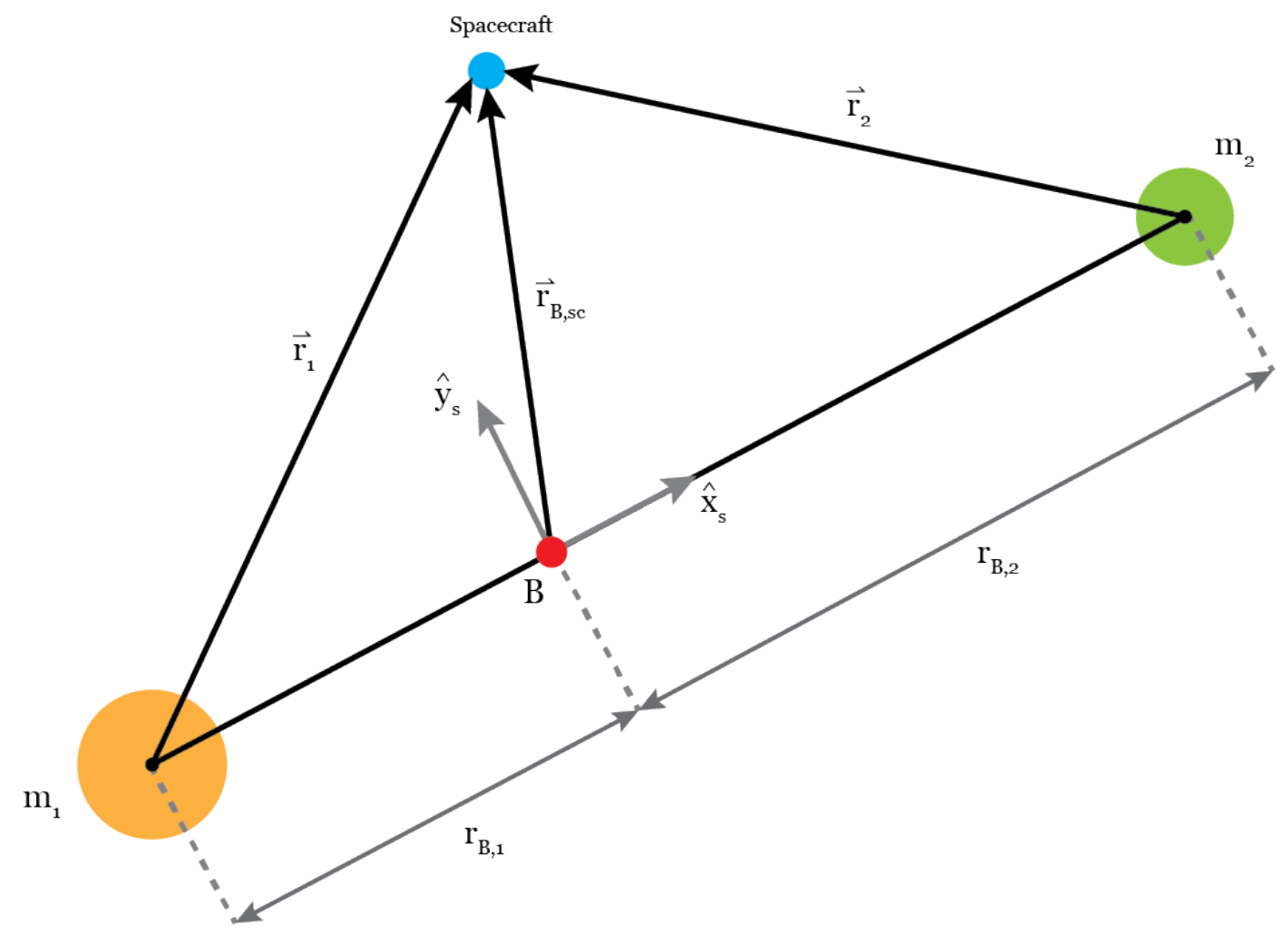

Figure 2.1: The Synodic Coordinate Frame used for the CRTBP. 
When working with the CRTBP it is advantageous to use non-dimensional units. This is accomplished by setting $m_{1}=1-\mu^{*}$ and $m_{2}=\mu^{*}$, where $\mu^{*}$ is defined as the mass ratio or three-body constant.

$$
\mu^{*}=\frac{m_{2}}{m_{1}+m_{2}}
$$

The distances from the primaries to the barycenter then become $r_{\mathrm{B}, 1}=-\mu^{*}$ and $r_{\mathrm{B}, 2}=1-\mu^{*}$. The magnitudes of $\overrightarrow{r_{1}}$ and $\overrightarrow{r_{2}}$ can be calculated using the following equations.

$$
\begin{gathered}
r_{1}=\sqrt{\left(x+\mu^{*}\right)^{2}+y^{2}+z^{2}} \\
r_{2}=\sqrt{\left(x+\mu^{*}-1\right)^{2}+y^{2}+z^{2}}
\end{gathered}
$$

The solved equations of motion for acceleration in $\mathrm{x}, \mathrm{y}$ and $\mathrm{z}$ for the system can then be seen in the equations below. For a full derivation of the equations refer to Vallado [17].

$$
\begin{gathered}
\ddot{x}=2 \dot{y}+x-\frac{\left(1-\mu^{*}\right)\left(x+\mu^{*}\right)}{r_{1}^{3}}-\frac{\mu^{*}\left(x+\mu^{*}-1\right)}{r_{2}^{3}} \\
\ddot{y}=-2 \dot{x}+y-\frac{\left(1-\mu^{*}\right) y}{r_{1}^{3}}-\frac{\mu^{*} y}{r_{2}^{3}} \\
\ddot{z}=-\frac{\left(1-\mu^{*}\right) z}{r_{1}^{3}}-\frac{\mu^{*} z}{r_{2}^{3}}
\end{gathered}
$$

\subsection{Lagrange Points}

There are five equilibrium points in the synodic coordinate frame where the acceleration and velocity due to the gravitational fields and system momentum are equal to zero. These locations in space are referred to as Lagrange or Libration points, and 
some of the most interesting and useful trajectories can be created in the proximity of these. There are three co-linear Lagrange points $\left(\mathrm{L}_{1}, \mathrm{~L}_{2}, \mathrm{~L}_{3}\right)$ that lie upon the $\mathrm{x}$ axis of the synodic coordinate frame, and two triangular Lagrange points $\left(\mathrm{L}_{4}, \mathrm{~L}_{5}\right)$ that lie where $\vec{r}_{1}$ and $\vec{r}_{2}$ are equal. For all the equilibrium points the $\mathrm{z}$ location must be equal to zero, or out of plane acceleration would cause oscillatory motion.

The $\mathrm{x}$ locations of the three co-linear points can be solved for using Equation 2.7 through Equation 2.9. The y coordinate for all of these points is zero since they lie along the $\mathrm{x}$ axis. $\mathrm{L}_{1}$ is defined as the point between the two primaries, $\mathrm{L}_{2}$ is the point just outside of the smaller primary, and $\mathrm{L}_{3}$ is the point on the opposite side of the larger primary from the smaller primary.

$$
\begin{aligned}
& L_{1}: x-\frac{\left(1-\mu^{*}\right)}{\left(x+\mu^{*}\right)^{2}}+\frac{\mu^{*}}{\left(x+\mu^{*}-1\right)^{2}}=0 \\
& L_{2}: x-\frac{\left(1-\mu^{*}\right)}{\left(x+\mu^{*}\right)^{2}}-\frac{\mu^{*}}{\left(x+\mu^{*}-1\right)^{2}}=0 \\
& L_{3}: x+\frac{\left(1-\mu^{*}\right)}{\left(x+\mu^{*}\right)^{2}}+\frac{\mu^{*}}{\left(x+\mu^{*}+1\right)^{2}}=0
\end{aligned}
$$

The $\mathrm{x}$ and $\mathrm{y}$ coordinates of the triangular points can then be found using Equation 2.10 and Equation 2.11.

$$
\begin{gathered}
L_{4}=\left(\frac{1}{2}-\mu^{*}, \frac{\sqrt{3}}{2}\right) \\
L_{5}=\left(\frac{1}{2}-\mu^{*}, \frac{-\sqrt{3}}{2}\right)
\end{gathered}
$$

A diagram of the Lagrange points for the Earth-Moon system can be seen in Figure 2.2. 


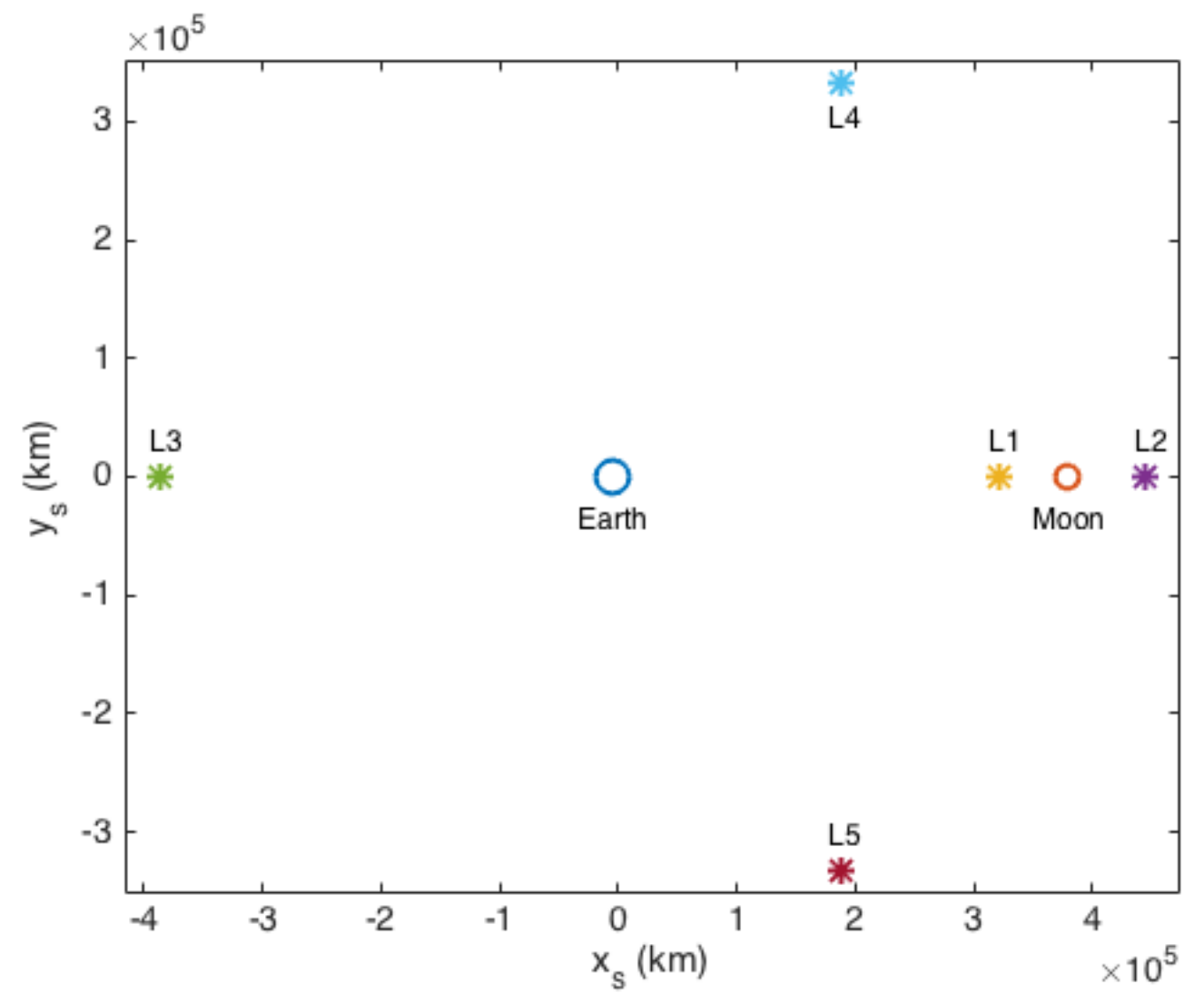

Figure 2.2: The five Lagrange points for the Earth-Moon system.

\section{$2.4 \quad$ Libration Orbits}

Libration orbits are orbits about a Lagrange point. There are multiple types of libration orbits, but some of the most common ones include Lyapunov, halo, and Lissajous. Lyapunov orbits are two dimensional and remain in the orbital plane of the primaries. Halo orbits are perfectly periodic three-dimensional orbits, while Lissajous orbits include periodic and quasi-periodic three-dimensional orbits [17]. In this case periodic means that a trajectory will retrace its path in the synodic reference frame, and quasi-periodic means the trajectory will trace a nearby path that is confined to a particular region of the synodic frame. Examples of a Lissajous orbit and a halo orbit can be seen in Figure 2.3 and Figure 2.4. For this study halo orbits were the 
only libration orbits considered in the solutions, and the computation of these will be covered in the next chapter.
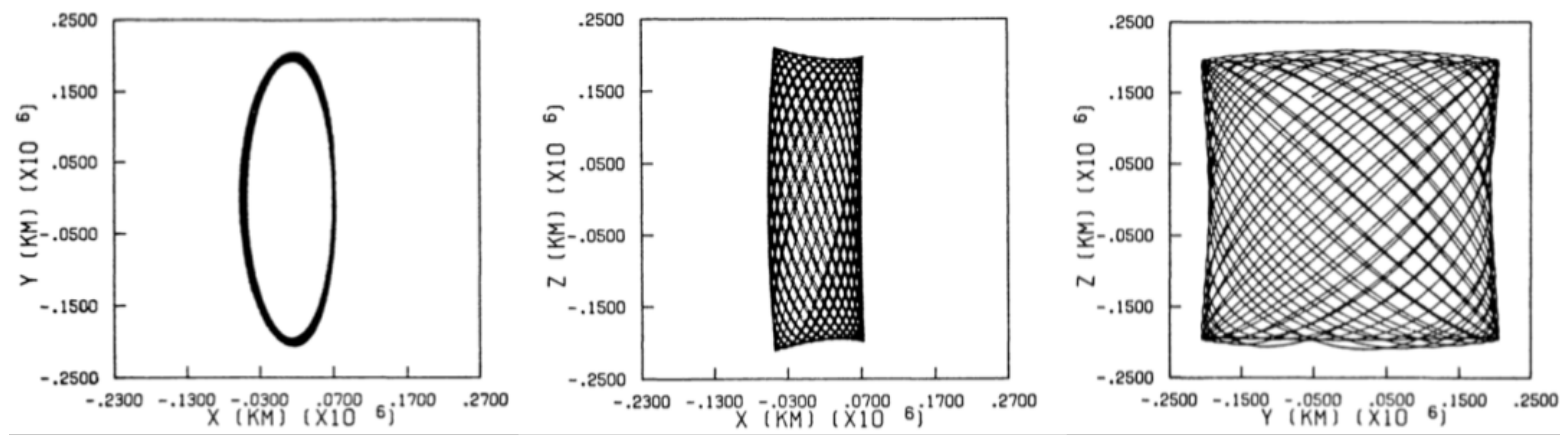

Figure 2.3: Example of Lissajous orbit about Sun-Earth $\mathrm{L}_{1}$ [6].
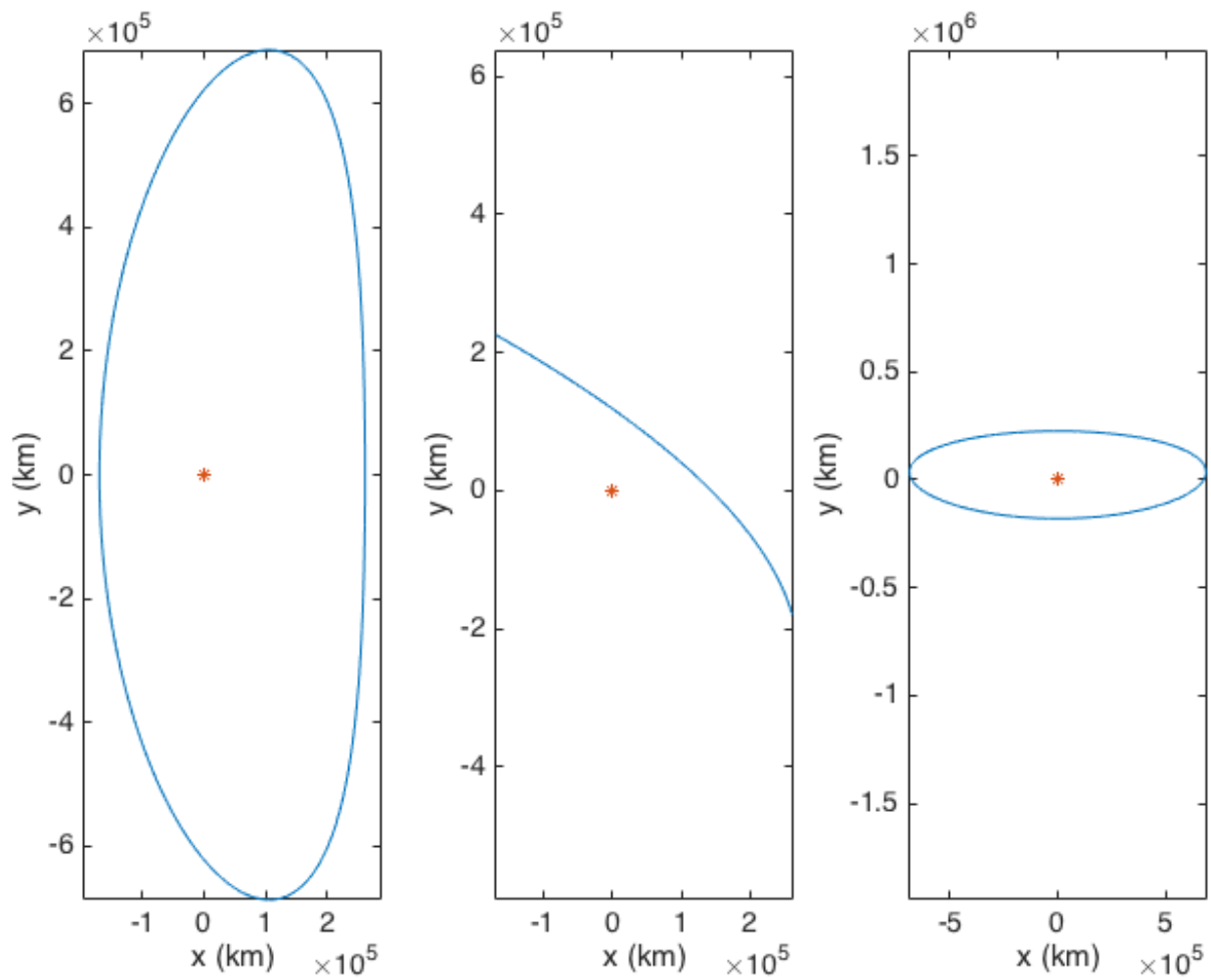

Figure 2.4: Example of a halo orbit about Sun-Earth $\mathrm{L}_{1}$. 


\subsection{Manifolds}

Manifolds are collections of trajectories that either approach or depart from a Lagrange point. Stable manifolds travel towards the point, and unstable manifolds travel away from the point. The stable and unstable manifolds for $\mathrm{L}_{2}$ of the EarthMoon system can be seen in Figure 2.5 and Figure 2.6.

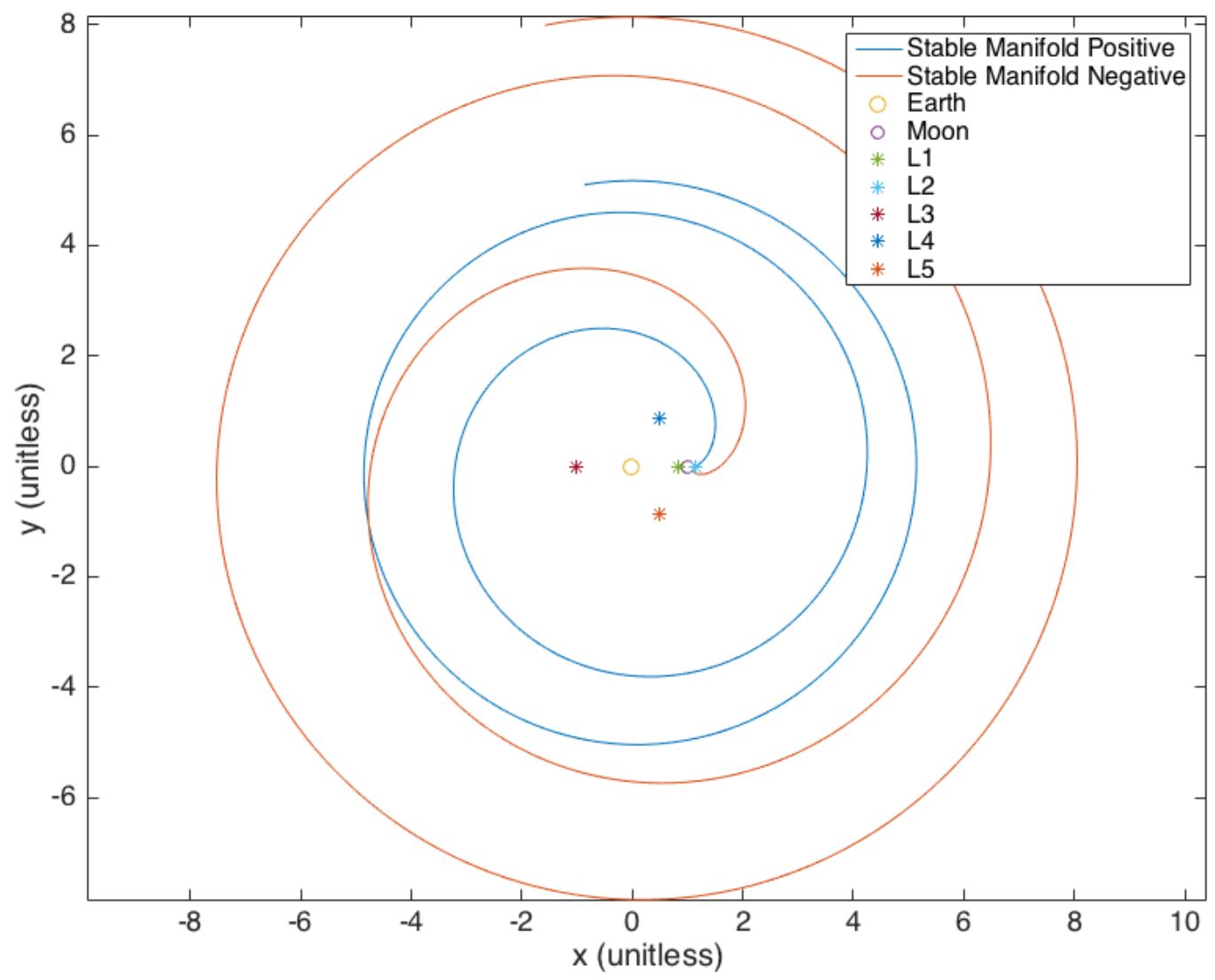

Figure 2.5: The stable manifolds approaching Earth-Moon $\mathrm{L}_{2}$. 


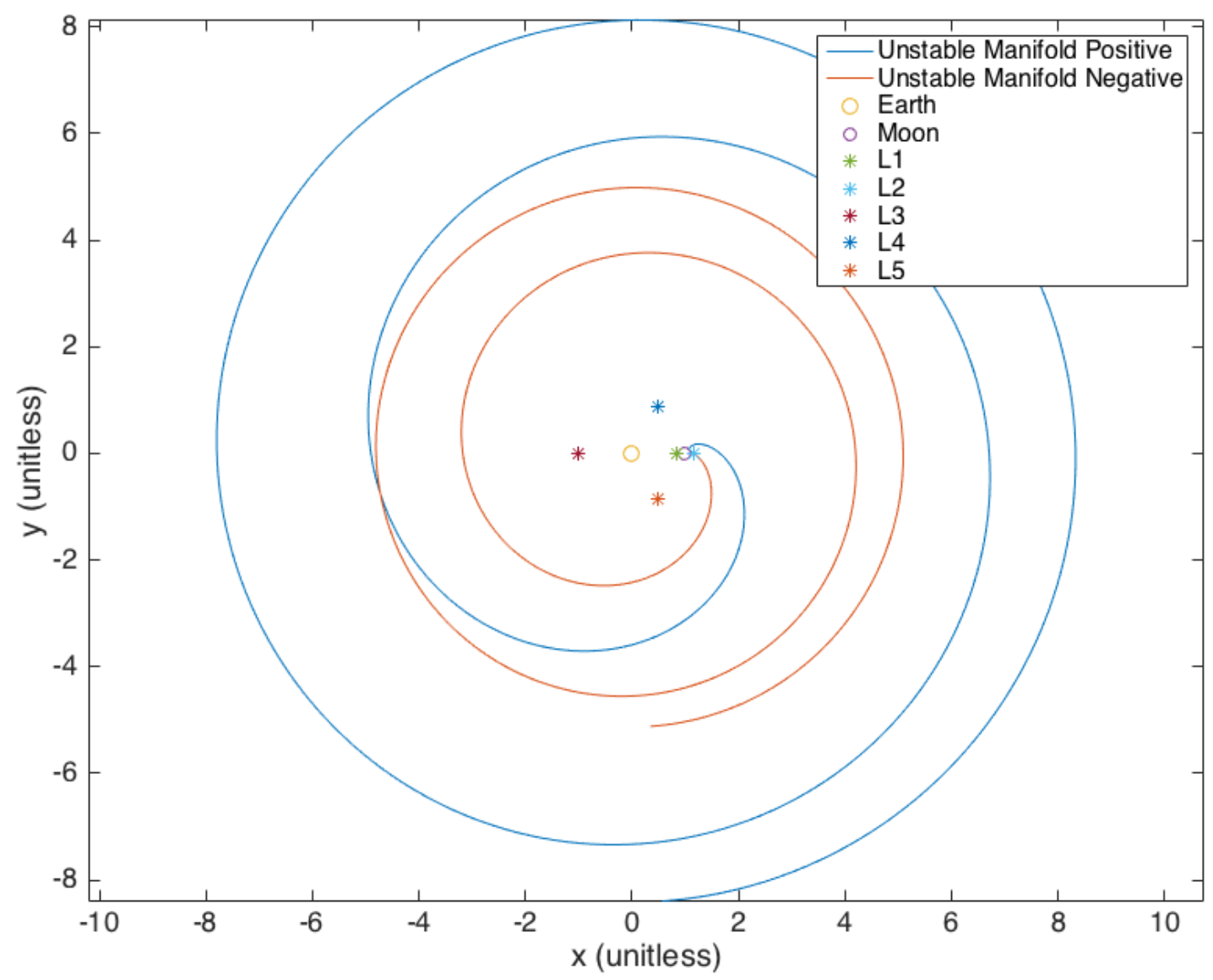

Figure 2.6: The unstable manifolds departing Earth-Moon $\mathrm{L}_{2}$.

The manifolds for a Lagrange point can be calculated by finding the eigenvalues of the Jacobian of the state vector at the Lagrange point. The corresponding eigenvectors are used to slightly perturb the state at the Lagrange point, and then propagating that state forward and backward in time will reveal the unstable and stable manifolds [17]. The state vector is defined as the following: 


$$
X=\left[\begin{array}{c}
x \\
y \\
z \\
\dot{x} \\
\dot{y} \\
\dot{z}
\end{array}\right]
$$

The Jacobian of the state vector is the following matrix:

$$
J=\left[\begin{array}{cccccc}
0 & 0 & 0 & 1 & 0 & 0 \\
0 & 0 & 0 & 0 & 1 & 0 \\
0 & 0 & 0 & 0 & 0 & 1 \\
\frac{\partial \ddot{x}}{\partial x} & \frac{\partial \ddot{x}}{\partial y} & \frac{\partial \ddot{x}}{\partial z} & 0 & 2 & 0 \\
\frac{\partial \ddot{y}}{\partial x} & \frac{\partial \ddot{y}}{\partial y} & \frac{\partial \ddot{y}}{\partial z} & -2 & 0 & 0 \\
\frac{\partial \ddot{z}}{\partial x} & \frac{\partial \ddot{z}}{\partial y} & \frac{\partial \ddot{z}}{\partial z} & 0 & 0 & 0
\end{array}\right]
$$

There are two imaginary pairs and one real pair of eigenvalues of the Jacobian for a Lagrange point. The eigenvector corresponding to the larger of the two real eigenvalues indicates the direction of the unstable manifold $\left(V^{U}\right)$, while the eigenvector corresponding to the smaller real eigenvalue indicates the direction of the stable manifold $\left(V^{S}\right)$.

The initial state vectors for the stable and unstable manifolds can then be calculated using

$$
\begin{aligned}
& X^{S}=X \pm \epsilon V^{S} \\
& X^{U}=X \pm \epsilon V^{U}
\end{aligned}
$$

where $\epsilon$ is a small perturbation. Then these state vectors can be propagated using the equations of motion for the CRTBP (Equation 2.4 through Equation 2.6). One 
must propagate the unstable manifold forwards in time and the stable one backwards in time.

Unstable periodic orbits about Lagrange points also have stable and unstable manifolds, but these form tubes of trajectories since a spacecraft can arrive or depart at any location on the orbit. A specific trajectory that a spacecraft departs or arrives on is just one of the many that make up the manifold. Invariant manifolds are tubes of trajectories that will always depart from or approach a halo orbit. An example of an unstable invariant manifold of a halo orbit about the Sun-Jupiter $\mathrm{L}_{2}$ point can be seen in Figure 2.7. The computation for invariant manifolds will be covered in section 3.4 .

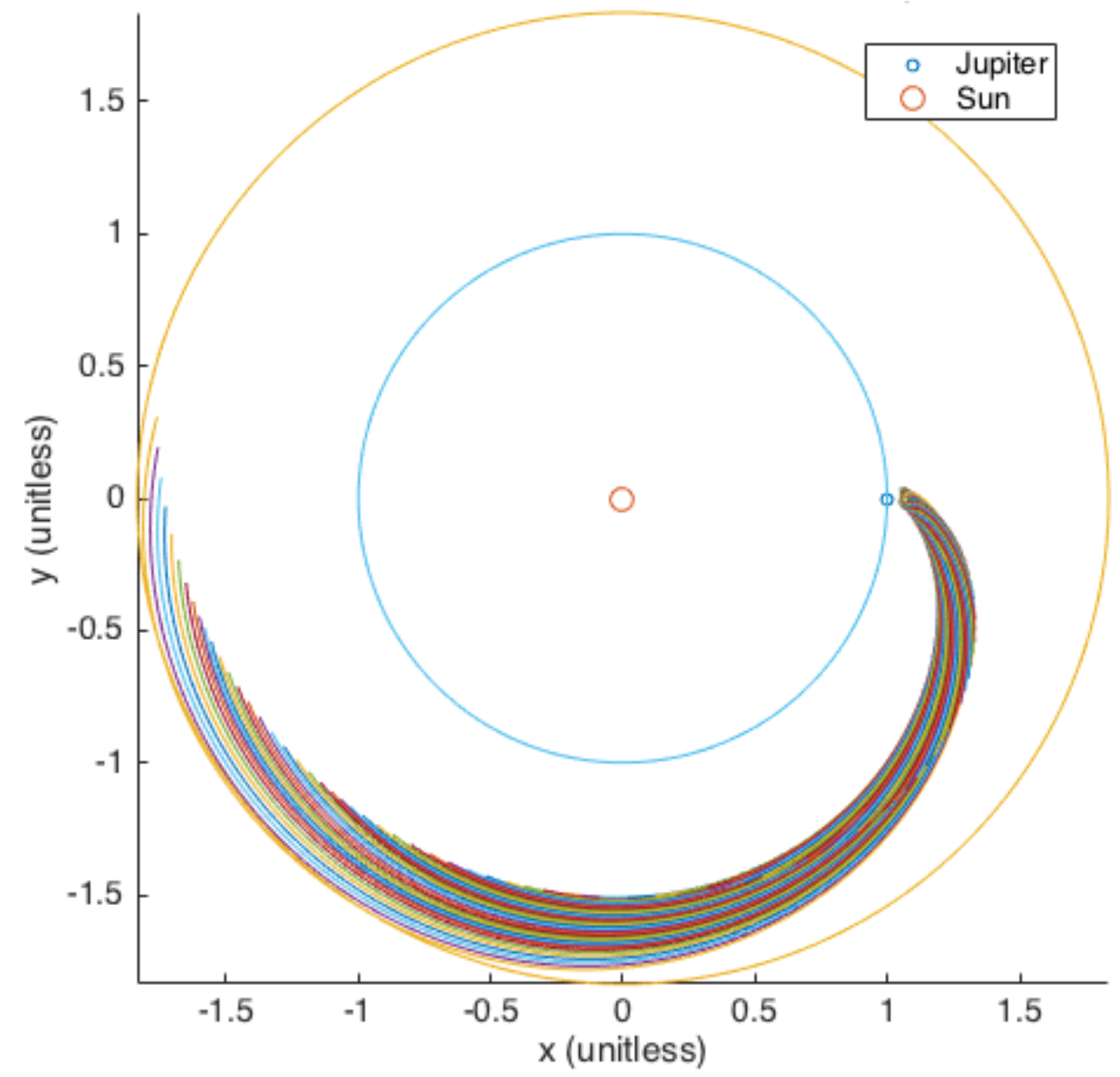

Figure 2.7: The unstable invariant manifold for a halo orbit about SunJupiter $\mathrm{L}_{2}$; z-amplitude of $300,000 \mathrm{~km}$. 
These manifolds can be used to a great advantage. Once a spacecraft is on one of the trajectories within the manifold it will continue to travel along it, with few corrections to combat perturbations. This means that a spacecraft can travel to or from a Lagrange point or periodic libration orbit with minimal $\Delta v$. 
Chapter 3

\section{HALO ORBITS AND INVARIANT MANIFOLDS}

\subsection{Overview}

Halo orbits are defined as perfectly periodic trajectories that orbit around a Lagrange point. By being perfectly periodic they repeat their path in space over and over again rather than diverging onto a new trajectory. These orbits can be very useful for many telescopes and Sun observing spacecraft, or can be used as gateways to interplanetary travel. For telescopes, $\mathrm{L}_{1}$ and $\mathrm{L}_{2}$ are advantageous since the Earth and the Sun always remain in the same location relative to the spacecraft. This means that thermal, imaging and power requirements that depend on the location of the sun are simplified. At $\mathrm{L}_{1}$ Sun observation missions have unobstructed views of the Sun at all times, and it is the perfect location to take pictures and collect data about solar wind and particles. Halo orbits are used at these locations so that spacecraft can stay orbiting along the same path rather than travelling out of the vicinity of the Lagrange point they are meant to be orbiting. The invariant manifolds of halo orbits can also be used to complete lower energy interplanetary transfers, as a spacecraft placed on one will always depart or approach the halo orbit along a specific path. An example of a halo orbit about $\mathrm{L}_{1}$ can be seen in Figure 3.1.

Halo orbits can be defined as being one of two classes: northern or southern. Northern halos spend the majority of their orbital period above the x-y plane, whereas

southern halos are mainly below the $\mathrm{x}-\mathrm{y}$ plane. The $\mathrm{y}-\mathrm{z}$ views of a northern and southern halo are shown in Figure 3.2. 


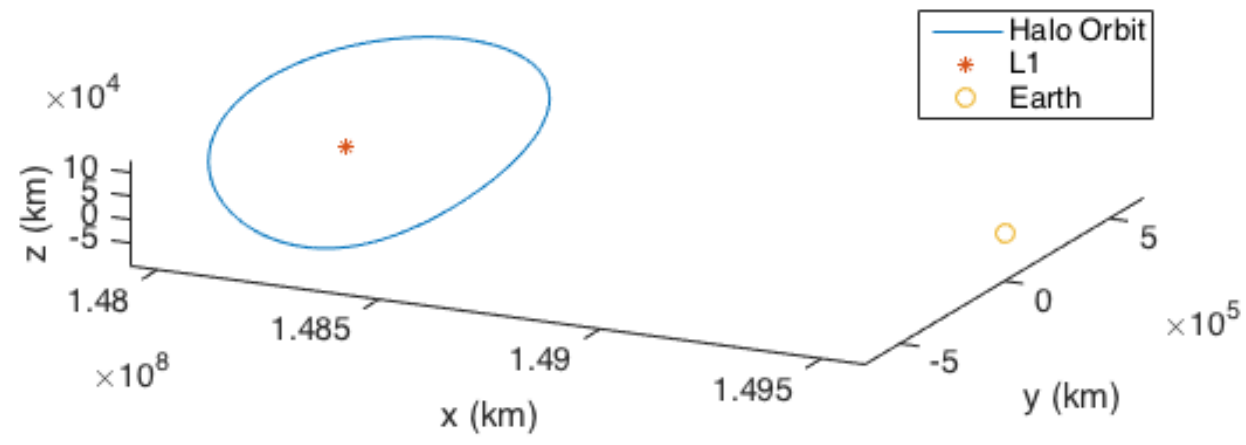

Figure 3.1: Halo orbit about Sun-Earth $\mathrm{L}_{1}$; z-amplitude of $110,000 \mathrm{~km}$.
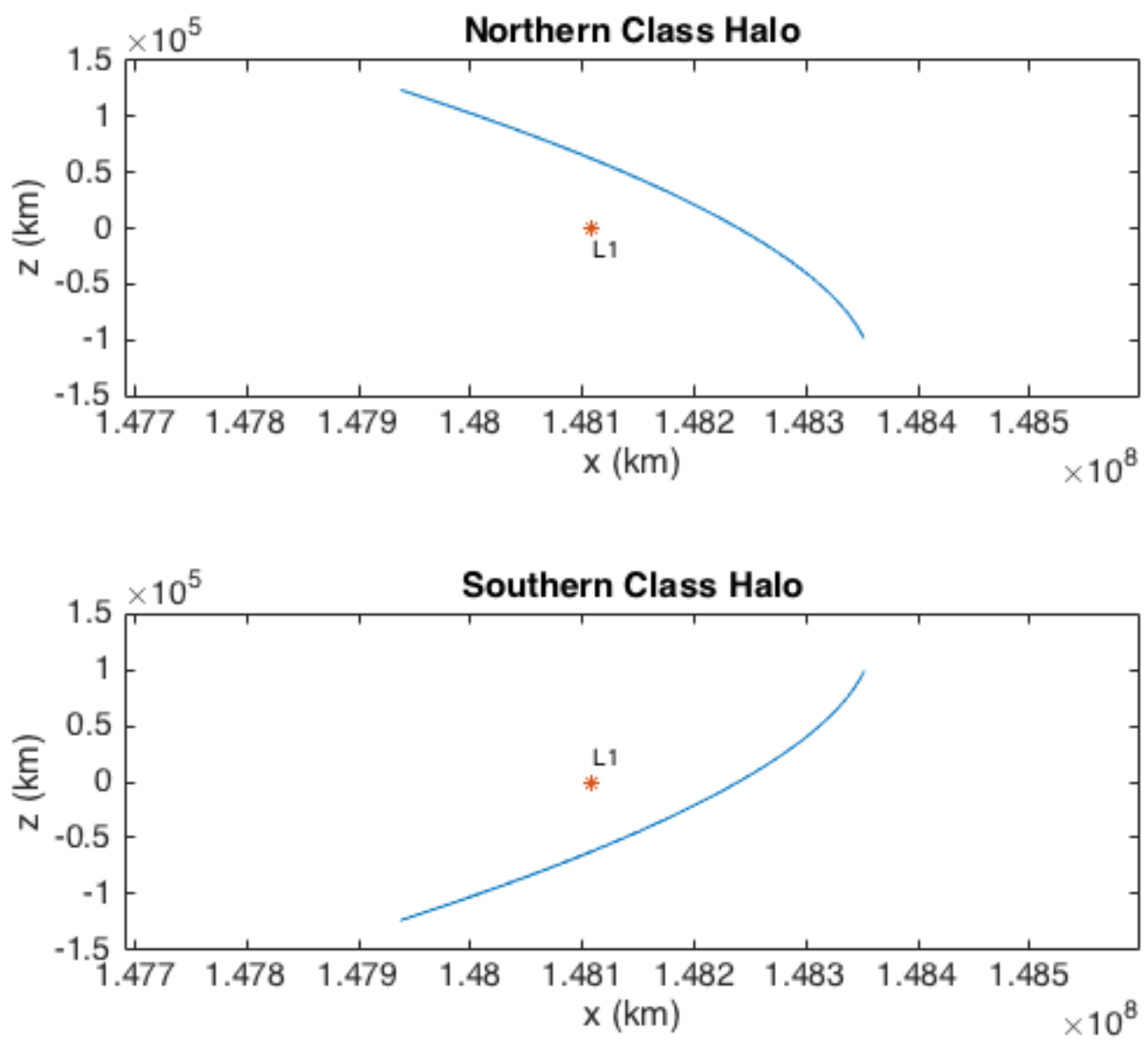

Figure 3.2: Example of a northern class halo (above) and southern class halo (below) about Sun-Earth $\mathrm{L}_{1}$; z-amplitude of 110,000 km. 
The size of halo orbits can be altered by changing the out-of-plane amplitude in the $\mathrm{z}$ direction $\left(\mathrm{A}_{\mathrm{z}}\right)$. By increasing this amplitude, one can increase the overall size of the halo orbit. A family of halo orbits about Sun-Earth $L_{1}$ with $A_{z}$ values ranging from 110,000 km to 1,100,000 km can be seen in Figure 3.3.

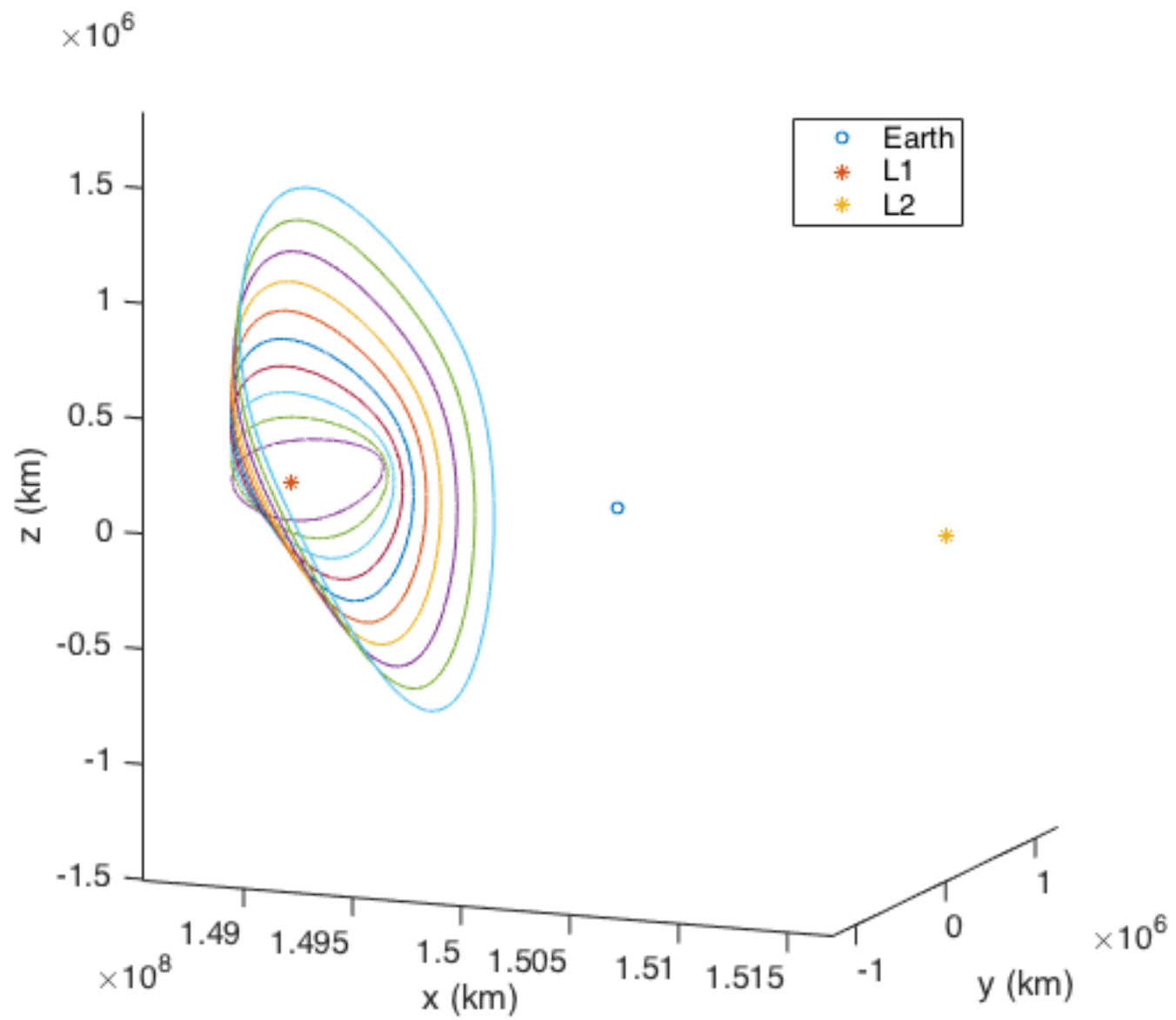

Figure 3.3: Family of northern class halo orbits about Sun-Earth $L_{1}$; zamplitude of 110,000 to $1,100,000 \mathrm{~km}$.

Solving the equations of motion in order to find a perfectly periodic orbit is very tricky. The best approximation that can be accomplished is estimating the trajectory of the halo orbit numerically. In order to do this an analytical solution is used approximate the trajectory of the desired size of halo orbit, and then this solution is used as a starting estimate for the numerical solution to iterate on.

The trajectories within the invariant manifolds are calculated once the halo orbit 
itself has been computed.

\subsection{Halo Orbit Analytical Solution}

The analytical solution here is thoroughly discussed and derived in Koon et al. in Chapter 6, "Halo Orbits and Their Computation" [7]. A brief summary of the method and the required equations to compute an initial estimate at the trajectory for a halo orbit will be supplied here. The equations were derived by Richardson using the Lindstedt-Poincare method.

First, the inputs required in order to calculate a halo orbit will be discussed. The system of celestial bodies must be chosen, so that $\mathrm{m}_{1}$ is the larger primary body and $\mathrm{m}_{2}$ is the smaller primary body. One should also know which Lagrange point the halo orbit should be centered around. Since most halos are only considered around $\mathrm{L}_{1}$ or $\mathrm{L}_{2}$, those will be the only solutions covered here. $A_{z}$ is the desired amplitude in the z-direction in kilometers and $\phi$ is the phase angle in radians. The orbit must also be specified as a northern or southern class of halo.

First the distance from the Lagrange point to the smaller primary is calculated. $\gamma_{1}$ is the distance for $\mathrm{L}_{1}$ and $\gamma_{2}$ is for $\mathrm{L}_{2}$. In these equations the variables $L_{1}$ and $L_{2}$ are the Lagrange point locations calculated using Equation 2.7 and Equation 2.8.

$$
\begin{aligned}
& \gamma_{1}=\left|1-\mu^{*}-L_{1}\right| \\
& \gamma_{2}=\left|L_{2}-1+\mu^{*}\right|
\end{aligned}
$$

Then, there are four c coefficients that are computed using the following equations, where $\mathrm{n}$ designates the number of the coefficient. The equations used differ slightly depending on whether the orbit is about $\mathrm{L}_{1}$ or $\mathrm{L}_{2}$, since the two points lie on opposite sides of the smaller primary body. If the orbit is about $\mathrm{L}_{1}$ Equation 3.3 is used, and 
for $\mathrm{L}_{2}$ Equation 3.4 is used.

$$
\begin{gathered}
L_{1}: c_{n}=\frac{1}{\gamma^{3}\left(1-\gamma^{n+1}\right)}\left(\mu^{*}+(-1)^{n}\left(1-\mu^{*}\right) \gamma^{n+1}\right. \\
L_{2}: c_{n}=\frac{1}{\gamma^{3}\left(1+\gamma^{n+1}\right)}\left(-1^{n} \mu^{*}+(-1)^{n}\left(1-\mu^{*}\right) \gamma^{n+1}\right.
\end{gathered}
$$

Next, $\omega_{p}$ and $k$ will need to be solved for. $\omega_{p}$ is one of the imaginary eigenvalues of the solution to the characteristic equation, and $k$ is the constant that relates the amplitude in $\mathrm{x}$ to the amplitude in $\mathrm{y}$.

$$
\begin{gathered}
\omega_{p}=\sqrt{2-c_{2}+\left(\frac{\left.9 c_{2}^{2}-8 c_{2}\right)}{2}\right)^{1 / 2}} \\
k=\frac{\omega_{p}^{2}+1+2 c_{2}}{2 \omega_{p}}
\end{gathered}
$$

The equations of motion for the analytical solution are very long and complicated, so many of the terms in them have been grouped into constants. All of the following equations are for the constants necessary to calculate the position and velocity vectors for the points in the halo orbit.

$$
\begin{gathered}
d_{1}=\frac{3 \omega_{p}^{2}}{k}\left(k\left(6 \omega_{p}^{2}-1\right)-2 \omega_{p}\right) \\
d_{2}=\frac{8 \omega_{p}^{2}}{k}\left(k\left(11 \omega_{p}^{2}-1\right)-2 \omega_{p}\right) \\
a_{21}=\frac{3 c_{3}\left(k^{2}-2\right)}{4\left(1+2 c_{2}\right)} ; \\
a_{22}=\frac{3 c_{3}}{4\left(1+2 c_{2}\right)}
\end{gathered}
$$




$$
\begin{aligned}
& a_{23}=\frac{-3 c_{3} \omega_{p}}{4 k d_{1}}\left(3 k^{3} \omega_{p}-6 k\left(k-\omega_{p}\right)+4\right) \\
& a_{24}=\frac{-3 c_{3} \omega_{p}}{4 k d_{1}}\left(2+3 k \omega_{p}\right) \\
& b_{21}=\frac{-3 c_{3} \omega_{p}}{2 d_{1}}\left(3 k \omega_{p}-4\right) \\
& b_{22}=\frac{-3 c_{3} \omega_{p}}{d 1} \\
& d_{21}=\frac{-c_{3}}{2 \omega_{p}^{2}} \\
& a_{31}=\frac{-9 \omega_{p}}{4 d_{2}}\left(4 c_{3}\left(k a_{23}-b_{21}\right)+k c_{4}\left(4+k^{2}\right)\right) \\
& +\frac{9 \omega_{p}^{2}+1-c_{2}}{2 d_{2}}\left(3 c_{3}\left(2 a_{23}-k b_{21}\right)+c_{4}\left(2+3 k^{2}\right)\right) \\
& a_{32}=\frac{-9 \omega_{p}}{4 d_{2}}\left(4 c_{3}\left(3 k a_{24}-b_{22}\right)+k c_{4}\right) \\
& -\frac{3}{2 d_{2}}\left(9 \omega_{p}^{2}+1-c_{2}\right)\left(c_{3}\left(k b_{22}+d_{21}-2 a_{24}\right)-c_{4}\right) \\
& b_{31}=\frac{3}{8 d_{2}} 8 \omega_{p}\left(3 c_{3}\left(k b_{21}-2 a_{23}\right)-c_{4}\left(2+3 k^{2}\right)\right) \\
& +\frac{3}{8 d_{2}}\left(\left(9 \omega_{p}^{2}+1+2 c_{2}\right)\left(4 c_{3}\left(k a_{23}-b_{21}\right)+k c_{4}\left(4+k^{2}\right)\right)\right) \\
& b_{32}=\frac{9 \omega_{p}}{d_{2}}\left(c_{3}\left(k b_{22}+d_{21}-2 a_{24}\right)-c_{4}\right)+\frac{3\left(9 \omega_{p}^{2}+1+2 c_{2}\right)}{8 d_{2}}\left(4 c_{3}\left(k a_{24}-b_{22}\right)+k c_{4}\right) \\
& d_{31}=\frac{3}{64 \omega_{p}^{2}}\left(4 c_{3} a_{24}+c_{4}\right) \\
& d_{32}=\frac{3}{64+\omega_{p}^{2}}\left(4 c_{3}\left(a_{23}-d_{21}\right)+c_{4}\left(4+k^{2}\right)\right)
\end{aligned}
$$


In order to compute a valid halo orbit, the amplitudes in $\mathrm{x}$ and $\mathrm{y}$ must be calculated based on the amplitude in z. The $s$ and $l$ constants are used to find the correct values of these amplitudes given $A_{z}$.

$$
\begin{gathered}
s_{1}=\left(2 \omega_{p}\left(\omega_{p}\left(1+k^{2}\right)-2 k\right)\right)^{-1} \\
\times\left(\frac{3}{2} c_{3}\left(2 a_{21}\left(k^{2}-2\right)-a_{23}\left(k^{2}+2\right)-2 k b_{21}\right)-\frac{3}{8} c_{4}\left(3 k^{4}-8 k^{2}+8\right)\right) \\
s_{2}=\left(2 \omega_{p}\left(\omega_{p}\left(1+k^{2}\right)-2 k\right)\right)^{-1} \\
\times\left(\frac{3}{2} c_{3}\left(2 a_{22}\left(k^{2}-2\right)+a_{24}\left(k^{2}+2\right)+2 k b_{22}+5 d_{21}\right)+\frac{3}{8} c_{4}\left(12-k^{2}\right)\right) \\
l_{1}=\frac{-3}{2} c_{3}\left(2 a_{21}+a_{23}+5 d_{21}\right)-\frac{3}{8} c_{4}\left(12-k^{2}\right)+2 \omega_{p}^{2} s_{1} \\
l_{2}=\frac{3}{2} c_{3}\left(a_{24}-2 a_{22}\right)+\frac{9}{8} c_{4}+2 \omega_{p}^{2} s_{2}
\end{gathered}
$$

Now using the $s$ and $l$ terms and a correction term, $\Delta$, the amplitude in $\mathrm{x}$ can be calculated. First, $A_{z}$ must be converted into canonical units by dividing it by one Distance Unit (the distance between the two primary bodies, $D U$ ) and then multiplied by $\gamma$ for the equations of motion.

$$
\begin{gathered}
A_{z}(\text { canonical })=A_{z}(k m) \frac{\gamma}{D U} \\
\Delta=\omega_{p}^{2}-c_{2} \\
A_{x}=\sqrt{\frac{-l_{2} A_{z}^{2}-\Delta}{l_{1}}}
\end{gathered}
$$

In order to remove secular terms from the solution a frequency connection term, $\nu$, and a new independent variable, $\tau$ are introduced. These can be found using Equation 3.29 and Equation 3.30.

$$
\nu=1+s_{1} A_{x}^{2}+s_{2} A_{z}^{2}
$$




$$
\tau=\nu t
$$

In the the equation for $\tau, \mathrm{t}$ is time in canonical units. To find the entire trajectory of the halo orbit, the the positions and velocities from time 0 until the period of the orbit $(T)$ will need to be calculated. This means $\tau$ will be a vector of times running from 0 to $T$ multiplied by the $\nu$ variable. The period of the halo orbit in canonical units can be estimated as:

$$
T=\frac{2 \pi}{\omega_{p} \nu}
$$

$\delta_{m}$ and $\tau_{1}$ are the final terms needed before the position and velocity are calculated. In the equation for $\delta_{m}, \mathrm{~m}=1$ for a northern halo and 3 for a southern halo.

$$
\begin{gathered}
\delta_{m}=2-m \\
\tau_{1}=\omega_{p} \tau+\phi
\end{gathered}
$$

Then motion in the halo orbit can be solved for at each point in the halo orbit by calculating position and velocity for every value of $\tau$.

Finally, the equations for position and velocity are as follow.

$$
\begin{gathered}
x=\gamma\left(a_{21} A_{x}^{2}+a_{22} A_{z}^{2}-A_{x} \cos \left(\tau_{1}\right)+\left(a_{23} A_{x}^{2}-a_{24} A_{z}^{2}\right) \cos \left(2 \tau_{1}\right)+\left(a_{31} A_{x}^{3}-a_{32} A_{x} A_{z}^{2}\right) \cos \left(3 \tau_{1}\right)\right) \\
y=\gamma\left(k A_{x} \sin \left(\tau_{1}\right)+\left(b_{21} A_{x}^{2}-b_{22} A_{z}^{2}\right) \sin \left(2 \tau_{1}\right)+\left(b_{31} A_{x}^{3}-b_{32} A_{x} A_{z}^{2}\right) \sin \left(3 \tau_{1}\right)\right) \\
z=\gamma\left(\delta_{m} A_{z} \cos \left(\tau_{1}\right)+\delta_{m} d_{21} A_{x} A_{z}\left(\cos \left(2 \tau_{1}\right)-3\right)+\delta_{m}\left(d_{32} A_{z} A_{x}^{2}-d_{31} A_{z}^{3}\right) \cos \left(3 \tau_{1}\right)\right) \\
\dot{x}=\gamma\left(\omega_{p} \nu A_{x} \sin \left(\tau_{1}\right)-2 \omega_{p} \nu\left(a_{23} A_{x}^{2}-a_{24} A_{z}^{2}\right) \sin \left(2 \tau_{1}\right)-3 \omega_{p} \nu\left(a_{31} A_{x}^{3}-a_{32} A_{x} A_{z}^{2}\right) \sin \left(3 \tau_{1}\right)\right)
\end{gathered}
$$


$\dot{y}=\gamma\left(\omega_{p} \nu k A_{x} \cos \left(\tau_{1}\right)+2 \omega_{p} \nu\left(b_{21} A_{x}^{2}-b_{22} A_{z}^{2}\right) \cos \left(2 \tau_{1}\right)+3 \omega_{p} \nu\left(b_{31} A_{x}^{3}-b_{32} A_{x} A_{z}^{2}\right) \cos \left(3 \tau_{1}\right)\right)$

$\dot{z}=\gamma\left(-\omega_{p} \nu \delta_{m} A_{z} \sin \left(\tau_{1}\right)-2 \omega_{p} \nu \delta_{m} d_{21} A_{x} A_{z} \sin \left(2 \tau_{1}\right)-3 \omega_{p} \nu \delta_{m}\left(d_{32} A_{z} A_{x}^{2}-d_{31} A_{z}^{2}\right) \sin \left(3 \tau_{1}\right)\right)$

Note that these equations are solved assuming the Lagrange point is the origin of the frame. In order to move the location of the halo orbit to be in the correct location in the synodic frame, the distance from the barycenter to the Lagrange point is added to all of the $\mathrm{x}$ positions.

While the analytical solution to finding halo orbit trajectories does get very close to an actual answer for the initial state, if only this method is used, the trajectory will diverge from the desired periodic one very quickly. When the initial state of the halo is propagated using the the equations of motion for the CRTBP, it is clear that the numerical solution is necessary to find an accurate halo orbit (see Figure 3.4). The analytical solution may not work as a final answer, but it is required to find a starting estimate to iterate on for the numerical solution, so must still be calculated.

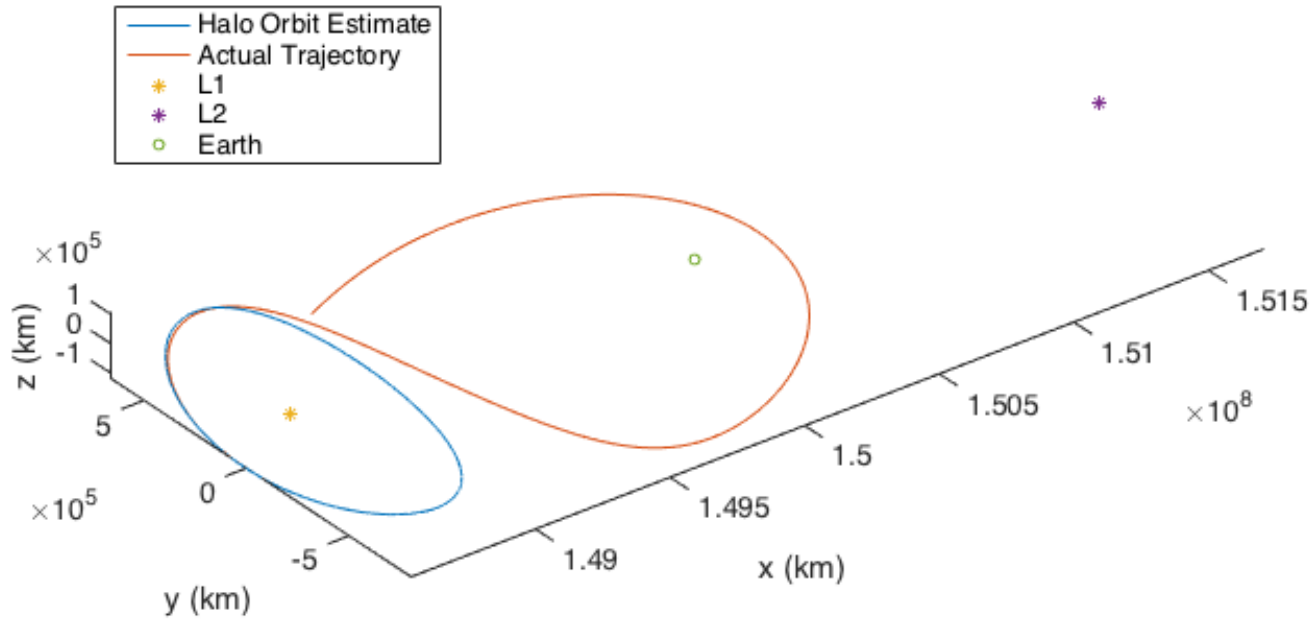

Figure 3.4: Analytically estimated halo orbit compared with the actual trajectory when propagated with the equations of motion. 


\subsection{Halo Orbit Numerical Solution}

The procedure detailed in Howell's research [4] is used to find the numerical solution for halo orbits. The numerical solution will require the state at time 0 of the analytical solution as an initial estimate to iterate on. At this point in the halo orbit, the position in $\mathrm{y}$ and the velocities in $\mathrm{x}$ and $\mathrm{z}$ must be equal zero, as this is the point where the halo orbit crosses the x-z plane. In order for the orbit to be periodic, the velocities in $\mathrm{x}$ and $\mathrm{z}$ must be equal to zero when the trajectory crosses the $\mathrm{x}-\mathrm{z}$ plane again (at half a period in time, $\mathrm{T} / 2$ ). The numerical solution covered by Howell, iterates to find a solution by slightly altering the initial state vector until $\dot{x}$ and $\dot{z}$ are sufficiently small when $\mathrm{y}=0$ again in the propagation of the orbit.

This calculation requires that the state transition matrix $(\Phi)$ is also calculated at every time step. The state transition matrix is used to relate the state vector $(X)$ at time 0 to the state vectors at all later time steps, so that:

$$
X\left(t_{i}\right)=\Phi\left(t_{0}+t_{i}, t_{0}\right) X\left(t_{0}\right)
$$

The state transition matrix must be propagated using an ODE solver just as the positions and velocities from the equations of motion are. The state transition matrix for halo orbits is a 6x6 matrix, and every one of the 36 elements is part of the state vector. This means that with the position and velocity in $\mathrm{x}, \mathrm{y}$ and $\mathrm{z}$, the state ends up being a 42x1 vector, represented by:

$$
X=\left[\begin{array}{lllllllllllll}
x & y & z & \dot{x} & \dot{y} & \dot{z} & \Phi_{1} & \Phi_{2} & \Phi_{3} & \Phi_{4} & \Phi_{5} & \Phi_{6} & ]^{T}
\end{array}\right.
$$

and the derivative of the state vector is:

$$
X=\left[\begin{array}{lllllllllllll} 
& \dot{x} & \dot{y} & \dot{z} & \ddot{x} & \ddot{y} & \ddot{z} & \dot{\Phi}_{1} & \dot{\Phi}_{2} & \dot{\Phi}_{3} & \dot{\Phi}_{4} & \dot{\Phi}_{5} & \dot{\Phi}_{6}
\end{array}\right]^{T}
$$

where the subscript on $\Phi$ indicates the row of the state transition matrix. 
The derivatives of position and velocity are known from the equations of motion of the CRTBP (Equation 2.4, Equation 2.5, and Equation 2.6). The derivative of the state transition matrix is found using Equation 3.43.

$$
\begin{gathered}
\dot{\Phi}=F \Phi \\
F=\left[\begin{array}{cc}
0 & I_{3} \\
U_{X X} & 2 \Omega
\end{array}\right]
\end{gathered}
$$

Here 0 represents a $3 \times 3$ matrix of zeros and $I_{3}$ is the $3 \times 3$ identity. $\Omega$ is the following matrix,

$$
\Omega=\left[\begin{array}{ccc}
0 & 1 & 0 \\
-1 & 0 & 0 \\
0 & 0 & 0
\end{array}\right]
$$

and $U_{X X}$ is equal to:

$$
U_{X X}=\left[\begin{array}{ccc}
U_{x x} & U_{x y} & U_{x z} \\
U_{y x} & U_{y y} & U_{y z} \\
U_{z x} & U_{z y} & U_{z z}
\end{array}\right]
$$

The terms inside of the $U_{X X}$ matrix are found by taking the second partial derivatives with respect to $\mathrm{x}, \mathrm{y}$ and $\mathrm{z}$ of $U$, which is equal to:

$$
U=\frac{1}{2}\left(x^{2}+y^{2}\right)+\frac{1-\mu^{*}}{\sqrt{\left(x+\mu^{*}\right)^{2}+y^{2}+z^{2}}}+\frac{\mu^{*}}{\sqrt{\left(x-1+\mu^{*}\right)^{2}+y^{2}+z^{2}}}
$$

This formula comes from the equations of motion for the CRTBP. The partial derivatives for the $U_{X X}$ matrix can be found using the following equations.

$$
\begin{gathered}
U_{x x}=\frac{1+\left(1-\mu^{*}\right)\left(-r_{1}^{2}+3\left(x+\mu^{*}\right)^{2}\right)}{r_{1}^{5}}+\frac{\mu^{*}\left(-r_{2}^{2}+3\left(x-1+\mu^{*}\right)^{2}\right)}{r_{2}^{5}} \\
U_{y y}=1+\frac{\left(1-\mu^{*}\right)\left(-r_{1}^{2}+3 y^{2}\right)}{r_{1}^{5}}+\frac{\mu^{*}\left(-r_{2}^{2}+3 y^{2}\right)}{r_{2}^{5}}
\end{gathered}
$$




$$
\begin{gathered}
U_{z z}=\frac{\left(1-\mu^{*}\right)\left(-r_{1}^{2}+3 z^{2}\right)}{r 1^{5}}+\frac{\mu^{*}\left(-r_{2}^{2}+3 z^{2}\right)}{r_{2}^{5}} \\
U_{x y}=U_{y x}=\frac{3 y\left(1-\mu^{*}\right)\left(x+\mu^{*}\right)}{r_{1}^{5}}+\frac{\mu^{*}\left(x-1+\mu^{*}\right)}{r_{2}^{5}} \\
U_{x z}=U_{z x}=\frac{3 z\left(\left(1-\mu^{*}\right)\left(x+\mu^{*}\right)\right.}{r_{1}^{5}}+\frac{\mu^{*}\left(x-1+\mu^{*}\right)}{r_{2}^{5}} \\
U_{y z}=U_{z y}=\frac{3 y z\left(\left(1-\mu^{*}\right)\right.}{r_{1}^{5}}+\frac{\mu^{*}}{r_{2}^{5}}
\end{gathered}
$$

The initial state vector for the first estimate of the numerical solution will consist of the position and velocity at time 0 from the analytical solution, and the $6 x 6$ identity as the state transition matrix. $y, \dot{x}$, and $\dot{z}$ should all be equal to zero at this point in time, so that the initial state vector not including $\Phi$ is:

$$
X_{0}=\left[\begin{array}{lllllll}
x_{0} & 0 & z_{0} & 0 & \dot{y}_{0} & 0
\end{array}\right]
$$

To consider the orbit periodic, $\dot{x}$ and $\dot{z}$ should be equal to zero when $y$ is equal to zero again at $\mathrm{T} / 2$, so the state vector without $\Phi$ at $\mathrm{T} / 2$ is:

$$
X_{T / 2}=\left[\begin{array}{lllllll}
x & 0 & z & 0 & \dot{y} & 0
\end{array}\right]
$$

It is not possible to find a solution where $\dot{x}$ and $\dot{z}$ are equal to exactly zero, so some tolerance is chosen to decide when the iterative scheme has reached a point that is satisfactory. The smaller the value of this tolerance, the more accurate the halo orbit approximation will be. $T o l=10^{-12}$ is a good starting point.

The following steps are required to find the solution:

1. Propagate the initial state vector using an ODE solver, such as ode45 in Matlab, until the position in $\mathrm{y}$ is equal to zero again $(\mathrm{T} / 2)$. 
2. Find the error in $\mathrm{x}$ and $\mathrm{z}$ velocities at $\mathrm{T} / 2(\delta \dot{x}$ and $\delta \dot{z})$.

$$
\begin{aligned}
& \delta \dot{x}=T o l-\dot{x} \\
& \delta \dot{z}=T o l-\dot{z}
\end{aligned}
$$

3. Calculate the change in the initial state required to reduce the error. It is only necessary to change two of the initial states. Since all the zero terms are correct those are left alone, and $\mathrm{x}$ and $\dot{y}$ are the only terms that will need to be altered. The initial position in $\mathrm{z}$ will remain fixed. $\delta x_{0}$ and $\delta \dot{y}_{0}$ are the values that will be used to change the initial state.

$$
\left[\begin{array}{c}
\delta x_{0} \\
\delta \dot{y}_{0}
\end{array}\right]=\left(\left[\begin{array}{ll}
\Phi_{41} & \Phi_{45} \\
\Phi_{61} & \Phi_{65}
\end{array}\right]-\frac{1}{v_{y}}\left[\begin{array}{l}
\ddot{x} \\
\ddot{z}
\end{array}\right]\left[\begin{array}{ll}
\Phi_{21} & \Phi_{25}
\end{array}\right]\right)^{-1}\left[\begin{array}{l}
\delta \dot{x} \\
\delta \dot{z}
\end{array}\right]
$$

Where the $\Phi$ terms are from the state transition matrix at $\mathrm{T} / 2$, and $\ddot{x}$ and $\ddot{z}$ are the accelerations at $\mathrm{T} / 2$.

4. Now, a new initial state is calculated by adding $\delta x_{0}$ and $\delta \dot{y}_{0}$ to the original initial state as in Equation 3.59 and Equation 3.60.

$$
\begin{aligned}
& x_{0}(\text { new })=x_{0}(\text { old })+\delta x_{0} \\
& \dot{y}_{0}(\text { new })=\dot{y}_{0}(\text { old })+\delta \dot{y}_{0}
\end{aligned}
$$

5. The new estimate for the initial state vector is now:

$$
X_{0}=\left[\begin{array}{lllllll}
x_{0}(n e w) & 0 & z_{0} & 0 & \dot{y}_{0}(n e w) & 0
\end{array}\right]
$$

Steps 1-4 are now repeated with the new estimate for the initial state.

This process is repeated until $\delta \dot{x}_{0}$ and $\delta \dot{z}_{0}$ are within the tolerance at $\mathrm{T} / 2$. Once the correct initial state is established, it can be propagated for one period and this 
yields the entire halo orbit. The comparison of the analytical solution and the numerical solution can be seen in Figure 3.5. The analytical solution is off by approximately $11,900 \mathrm{~km}$ in $\mathrm{x}$ position and $11.4 \mathrm{~m} / \mathrm{s}$ in $\mathrm{y}$ velocity for the initial state vector. This difference in the starting state means that the propagated analytical solution will not be periodic, while the numerical solution will.

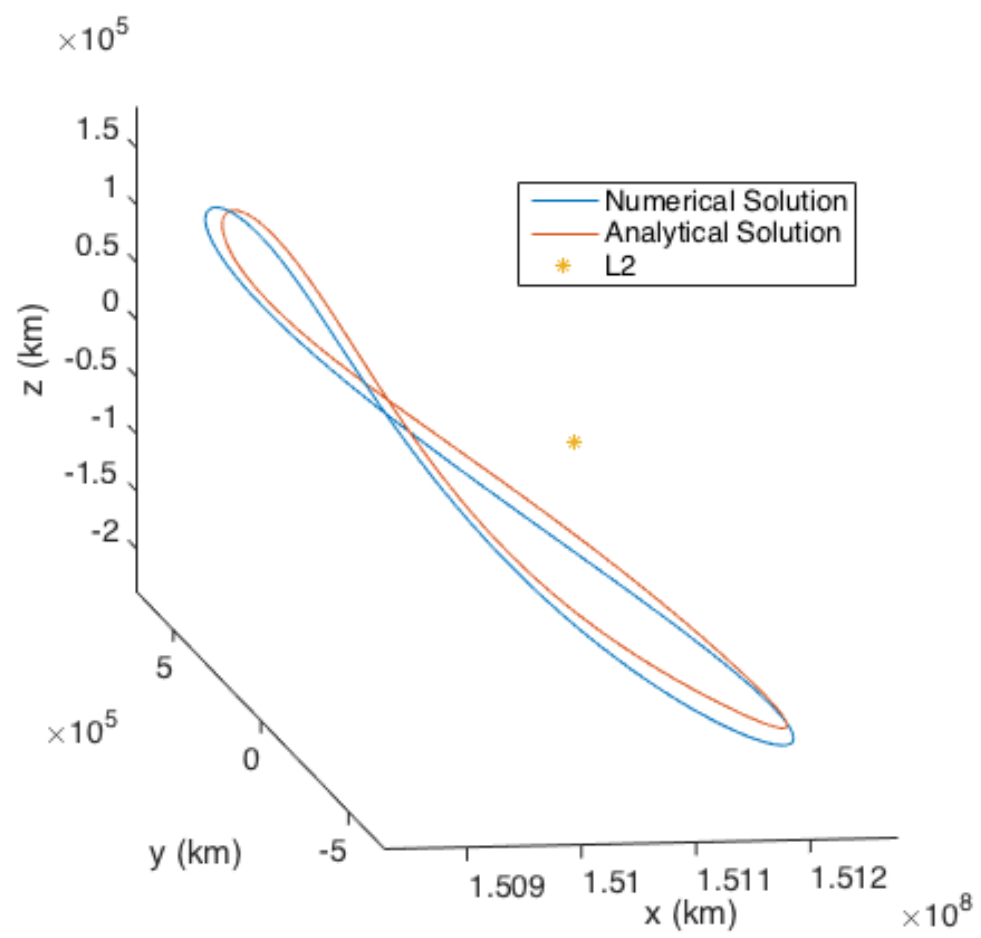

Figure 3.5: Comparison of the halo orbit trajectories generated by the numerical and analytical solutions.

\subsection{Invariant Manifolds}

Once all the points of the halo orbit have been calculated using the numerical solution, the trajectories within the invariant manifolds departing from each of those points can be computed. The invariant manifolds can be found by finding the eigenvalues of the Jacobian at each point along the halo orbit, but since this is computationally expensive it is more advantageous to use the monodromy matrix. The monodromy matrix is simply the state transition matrix after one period, or at time $\mathrm{T}$, and 
contains information about the stability of the entire orbit, so its eigenvalues can be used in place of the Jacobian eigenvalues.

$$
M=\Phi\left(t_{0}+T, t_{0}\right)
$$

Then the eigenvalues and corresponding eigenvectors of $M$ must be calculated. There are six eigenvalues of the monodromy matrix, but only the maximum and minimum real eigenvalues are required to find the invariant manifolds. The eigenvector corresponding to the smaller eigenvalue is used to calculate the stable manifold, while the eigenvector for the larger eigenvalue is used for the unstable manifold. Let $V^{S}$ denote the eigenvector for the stable manifold and $V^{U}$ be the eigenvector for the unstable one. These eigenvectors can then be found at every point along the halo using the state transition matrix at each time step, as is shown in Equation 3.63 and Equation 3.64. Here $i$ represents the point along the halo being evaluated.

$$
\begin{aligned}
& V_{i}^{S}=\Phi\left(t_{0}+t_{i}, t_{0}\right) V^{S} \\
& V_{i}^{U}=\Phi\left(t_{0}+t_{i}, t_{0}\right) V^{U}
\end{aligned}
$$

Now these eigenvectors can be used to find the initial conditions for the trajectories within the stable and unstable manifolds originating from each point of the halo $\left(X_{i}^{S}\right.$ and $\left.X_{i}{ }^{U}\right)$ with the following equations.

$$
\begin{aligned}
& X_{i}^{S}=X_{i} \pm \epsilon \frac{V_{i}^{S}}{\left|V_{i}^{S}\right|} \\
& X_{i}^{U}=X_{i} \pm \epsilon \frac{V_{i}^{U}}{\left|V_{i}^{U}\right|}
\end{aligned}
$$

Where $\mathrm{X}_{\mathrm{i}}$ represents the point along the halo and $\epsilon$ is a small number used to perturb the state on the halo. This value is usually around $1000 \mathrm{~km}$ for the SunEarth system (approximately $10^{-5}$ in canonical units).

The initial states for the invariant manifolds can be propagated using the equations of motion for the CRTBP. Since the unstable ones depart the halo orbit, they are 
propagated forward in time. But, the stable manifolds must be propagated backwards in time since the halo orbit is the final state. An example of the stable manifold for Sun-Jupiter $\mathrm{L}_{2}$ can be seen in Figure 3.6.

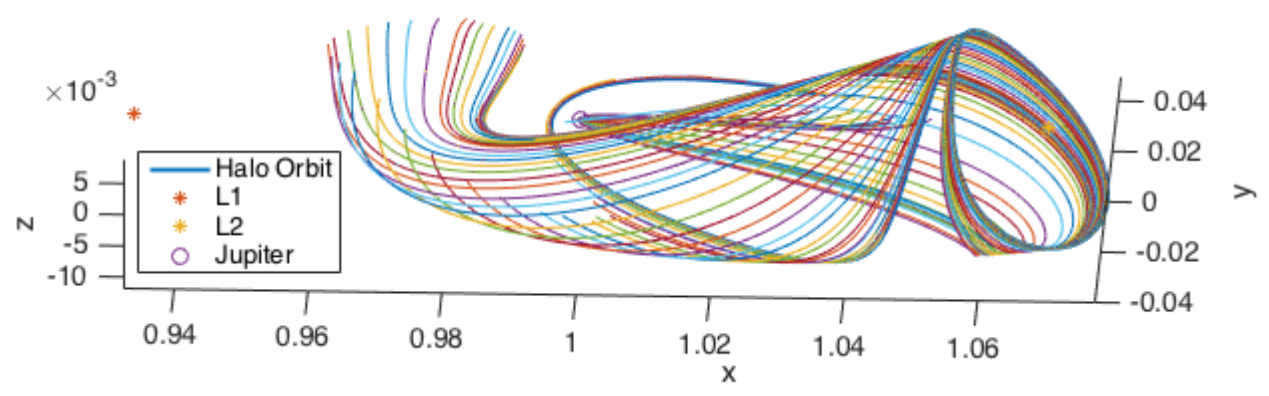

Figure 3.6: Example the stable invariant manifold for Sun-Jupiter $L_{2}$. 
Chapter 4

\section{MANEUVERS FOR INTERPLANETARY TRANSFERS}

\subsection{Overview}

There are multiple maneuvers required to use the invariant manifolds of halo orbits to complete an interplanetary transfer. First a spacecraft must depart from the Earth and reach a halo orbit about a Sun-Earth Lagrange point. This maneuver will require a transfer from a parking orbit about the Earth onto a trajectory within a the stable manifold of the halo orbit. There is no maneuver required to be inserted onto the halo orbit as the invariant manifold will eventually bring a spacecraft onto the halo. Then a spacecraft must leave the halo orbit on a trajectory within the unstable invariant manifold to return towards Earth for a transfer onto a hyperbolic escape trajectory. Then a maneuver from the trajectory within the unstable manifold onto

the hyperbolic escape trajectory must be completed, and a spacecraft will begin the journey on to the destination planet. Upon arrival, either a maneuver from the hyperbolic capture trajectory onto an invariant manifold of a halo orbit at the destination planet can be completed, or a flyby maneuver can be executed. This chapter will cover the methods for computing how to connect these different trajectories and the $\Delta v$ required for the maneuvers.

\subsection{Earth to Halo Transfer}

In order to use invariant manifolds to find interplanetary trajectories, first a transfer from Earth to a halo orbit about a Sun-Earth Lagrange point must be computed. The transfer will need to begin in a parking orbit about Earth, and then use a trajectory within the stable invariant manifold to travel to the halo. The only maneuver required 
will be the burn to get onto the manifold from the parking orbit. It is possible to direct inject onto a trajectory within the manifold, but this analysis will only consider a transfer from a parking orbit.

Once a desired halo orbit is chosen, and the invariant manifolds are calculated, the trajectories that pass closest to the Earth must be found. First, the trajectories within the stable invariant manifold are calculated for 300 evenly spaced points around the halo orbit, and the one that passes closest to the Earth is identified by finding the smallest difference in the position vector of the Earth and and all the position vectors of each trajectory within the manifold. Then the trajectories for 100 points on either side of the point within the halo corresponding to the trajectory with the closest approach are calculated; this will find the 200 trajectories within the manifold that pass the closest to the Earth. An example of these trajectories can be seen in Figure 4.1 for a halo orbit about $\mathrm{L}_{2}$.

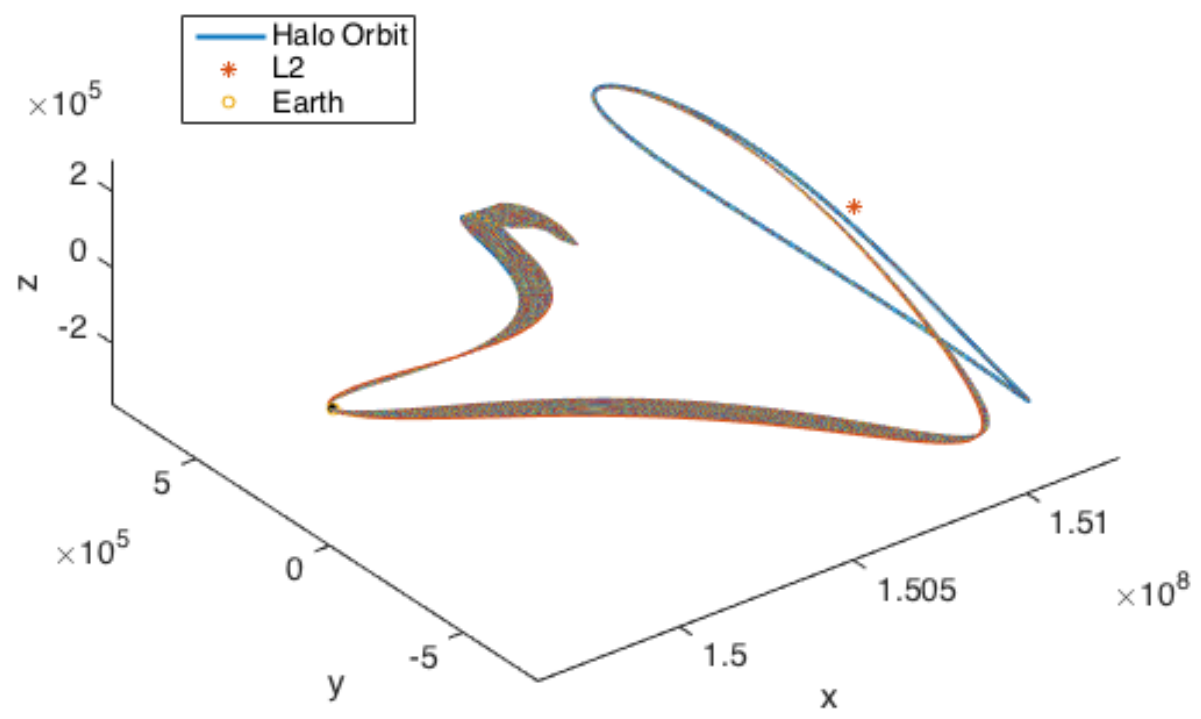

Figure 4.1: An example of the 200 trajectories within the stable invariant manifold that pass closest to Earth for a halo about $\mathrm{L}_{2}$.

Now, a design choice must be made to leave from a parking orbit with the lowest 
altitude, or to leave from any altitude parking orbit with the lowest $\Delta v$ for the maneuver onto the manifold. The disadvantage of using a larger parking orbit is that it requires more $\Delta v$ to get to the higher altitude for a launch vehicle. A trade will be run on the total $\Delta v$ for both of these parking orbit methods to see which is more optimal. To obtain the lowest altitude parking orbit, the trajectory passing closest to the Earth is identified, as well as the point within it that is the closest approach to the Earth. Then, the inclination $(i)$, Right Ascension of the Ascending Node (RAAN, $\Omega$ ), and argument of perigee $(\omega)$ are determined for the parking orbit, as well as the true anomaly $(\theta)$ at the intersection point. These orbital elements are determined by estimating what they would be based on the position and velocity vectors $\left(\vec{r}_{m}\right.$ and $\vec{v}_{m}$ ) of the closest approach point within the manifold. The equations to find these elements can be found in Appendix A and in Curtis [2].

The eccentricity of the parking orbit is zero since it is defined as circular. The radius of the orbit $\left(r_{\text {park }}\right)$ is simply the magnitude of the position vector for the closest approach point within the manifold trajectory. Then the magnitude of the specific angular momentum for a circular orbit $\left(h_{\text {park }}\right)$ can be calculated using Equation 4.1.

$$
h_{\text {park }}=\sqrt{\mu r_{\text {park }}}
$$

Now all of the orbital elements for the parking orbit have been calculated and can be used to find the velocity vector at the departure point onto the manifold trajectory from the parking orbit. The velocity vector is computed in the perifocal frame and is then rotated via a 3-1-3 rotation sequence using the angles of the inclination, RAAN, and argument of perigee to be in the translated synodic frame. The perifocal frame is centered at the focus of the orbit with the $x-y$ plane aligned with the plane of the orbit. The $\mathrm{x}$-axis points toward the periapsis point, and the $\mathrm{z}$ axis is normal to the orbital plane and parallel to the angular velocity vector, $\vec{h}$. Then the y-axis is $90^{\circ}$ away from the $\mathrm{x}$-axis and completes the right-handed coordinate system [2]. The 
equations used to obtain the velocity vector can be found in Appendix B.

Then the $\Delta v$ for the maneuver onto the manifold is calculated by finding the difference in velocities between the insertion point on the manifold trajectory and the velocity in the parking orbit at the intersection point.

$$
\Delta v_{p a r k}=\left|\vec{v}_{m}-\vec{v}_{p a r k}\right|
$$

If it is desired to leave from the parking orbit with the lowest $\Delta v$ for the maneuver onto the manifold, then the same process for finding the parking orbit is completed, but this time for all of the 200 trajectories passing closest to Earth. Then the $\Delta v$ is calculated for all the parking orbit options, and the solution with the lowest required $\Delta v$ is selected.

The different results for parking orbit altitudes and $\Delta v$ values for various halo orbit sizes can be seen in Table 4.1.

Table 4.1: $\Delta v$ and parking orbit altitudes for minimized parking orbit altitude and minimized $\Delta v$ for various halo orbit sizes.

\begin{tabular}{c|cc|cc} 
& \multicolumn{2}{|c|}{ Minimized Parking Orbit Altitude } & \multicolumn{2}{c}{ Minimized $\Delta v$} \\
\hline \hline Halo Orbit $\mathrm{A}_{z}$ & $\Delta v$ & Parking Orbit Altitude & $\Delta v$ & Parking Orbit Altitude \\
\hline $110,000 \mathrm{~km}$ & $2.40 \mathrm{~km} / \mathrm{s}$ & $5033 \mathrm{~km}$ & $1.54 \mathrm{~km} / \mathrm{s}$ & $20,091 \mathrm{~km}$ \\
$500,000 \mathrm{~km}$ & $3.17 \mathrm{~km} / \mathrm{s}$ & $285.1 \mathrm{~km}$ & $1.60 \mathrm{~km} / \mathrm{s}$ & $18,455 \mathrm{~km}$ \\
$800,000 \mathrm{~km}$ & $1.37 \mathrm{~km} / \mathrm{s}$ & $28,019 \mathrm{~km}$ & $1.00 \mathrm{~km} / \mathrm{s}$ & $54,305 \mathrm{~km}$
\end{tabular}

It is clear that larger and smaller halos do not have trajectories within the manifold that pass into Low Earth Orbit (LEO), but medium sized halos can get to parking orbits as low as $200 \mathrm{~km}$ in altitude. If a low parking orbit is a mission requirement, this would influence what sized halo should be chosen for the later transfers. Since this study is not limited to a specific parking orbit, it will use the transfer that results in the least total $\Delta v$ after launch into LEO. It can also be seen that leaving from 
higher parking orbits leads to a smaller burn onto the trajectory within the manifold. It is not immediately clear whether the total $\Delta v$ will be reduced by completing a Hohmann transfer from LEO to the higher parking orbit altitudes, where less $\Delta v$ is needed to get onto the manifold, or whether it is more advantageous to just complete one large maneuver onto a trajectory that can be reached from LEO. Therefore, calculations were run for both cases, to compare and see which concept of operations would reduce the total $\Delta v$. The results for the $500,000 \mathrm{~km}$ halo are shown in Table 4.2, and it is clear that the Hohmann transfer (assuming launch into $300 \mathrm{~km}$ parking orbit) increases the total $\Delta v$ significantly. The smaller $\Delta v$ for the transfer onto the manifold does not decrease with altitude enough for the Hohmann to be worth it. Therefore, only trajectories passing into LEO will be considered for the transfer to the halo from Earth. Also, the approximately $3 \mathrm{~km} / \mathrm{s}$ burn required to leave a LEO parking orbit to get onto a manifold matches the results seen in works by Howell et al. [5].

Table 4.2: Comparison of total $\Delta v$ to get to halo orbit using higher and lower altitude parking orbits for a halo orbit with $A_{z}$ equal to 500,000 km.

\begin{tabular}{cccc} 
Parking Orbit Altitude & Hohmann $\Delta v$ & Manifold Transfer $\Delta v$ & Total $\Delta v$ \\
\hline $285.1 \mathrm{~km}$ & $0 \mathrm{~km} / \mathrm{s}$ & $3.17 \mathrm{~km} / \mathrm{s}$ & $3.17 \mathrm{~km} / \mathrm{s}$ \\
$18,455 \mathrm{~km}$ & $3.37 \mathrm{~km} / \mathrm{s}$ & $1.60 \mathrm{~km} / \mathrm{s}$ & $4.97 \mathrm{~km} / \mathrm{s}$
\end{tabular}

Once a spacecraft is on the stable invariant manifold, theoretically it will not require anymore maneuvers, since the trajectory will lead it straight onto the halo, with no insertion $\Delta v$ cost [5]. In reality, there will be perturbations and orbital corrections, but they are not taken into account in this thesis. An example of the trajectory to get onto a $300,000 \mathrm{~km}$ halo is shown in Figure 4.2. A close-up view of the parking orbit at the Earth and the transfer point onto the manifold can be seen in Figure 4.3 


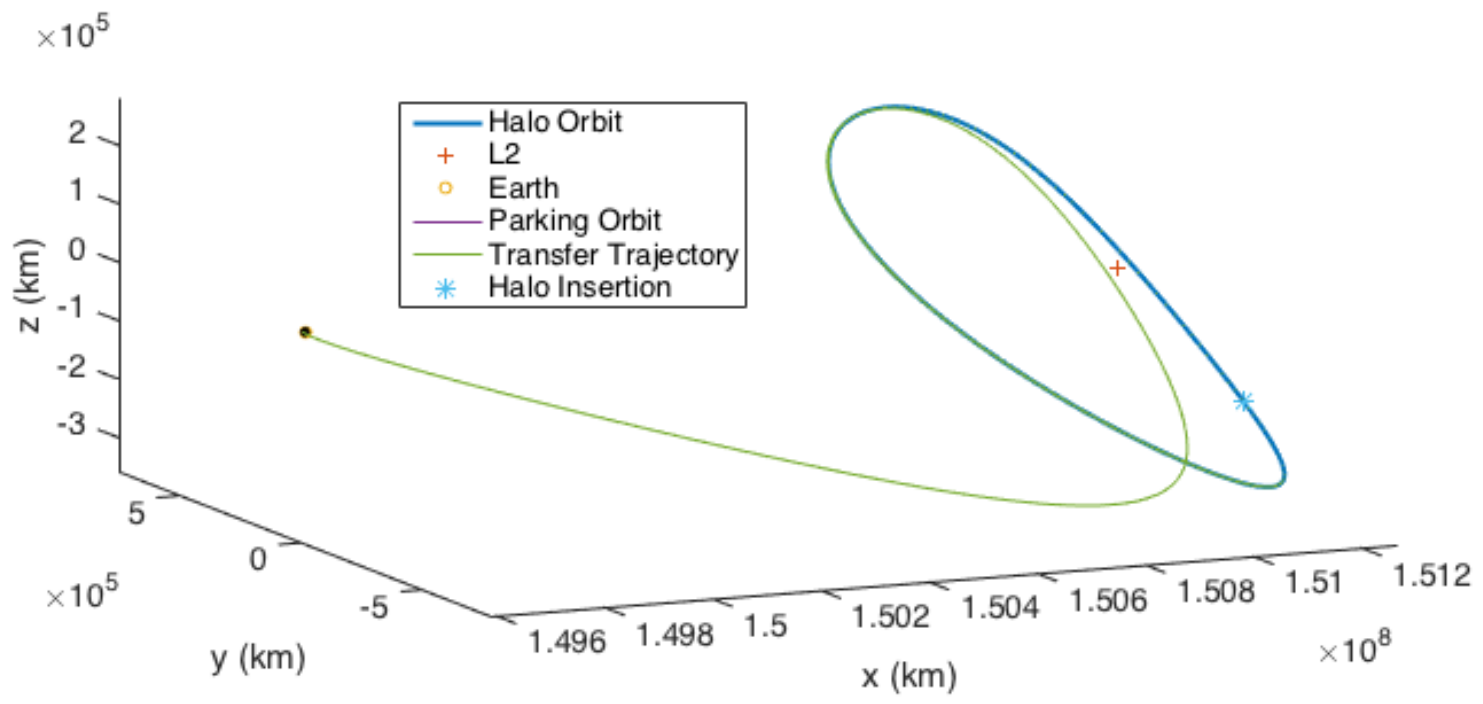

Figure 4.2: Example of transfer trajectory from Earth parking orbit to halo with $A_{z}$ equal to $300,000 \mathrm{~km}$.

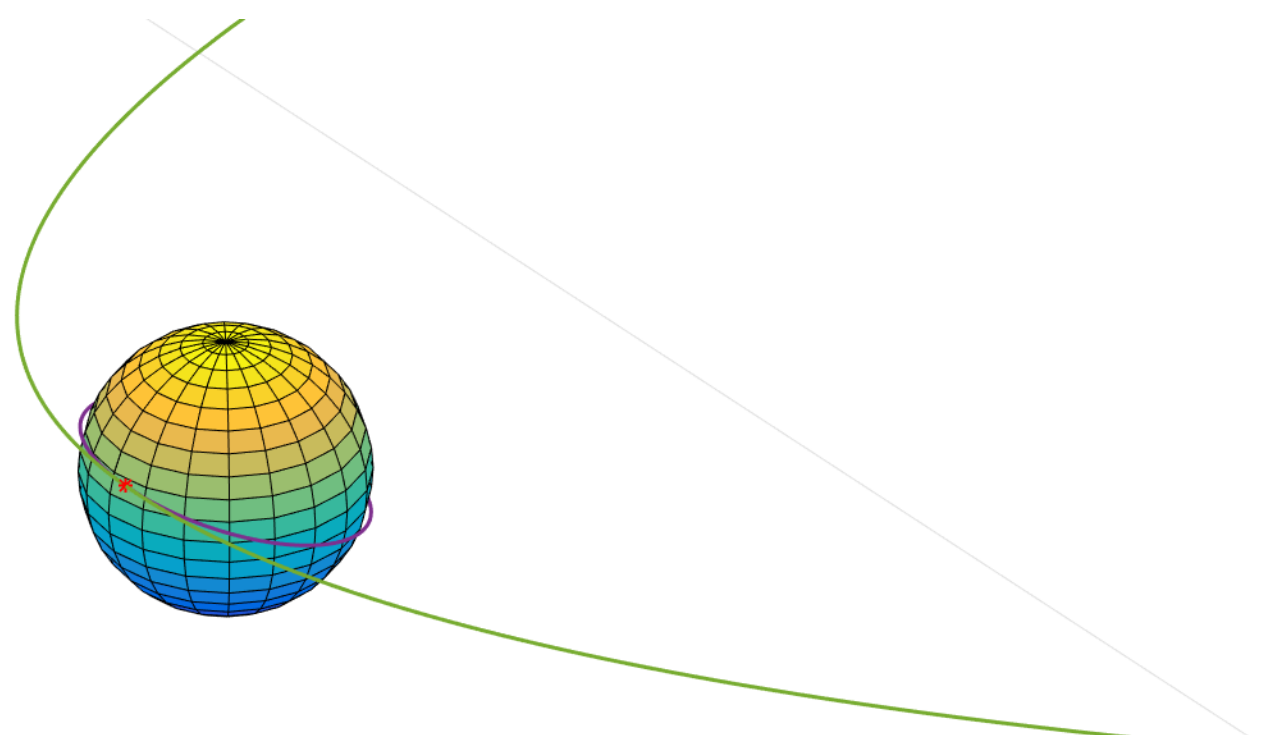

Figure 4.3: Close up view of transfer point from Earth parking orbit onto trajectory within the stable invariant manifold.

\subsection{Interplanetary Transfer}

Once a spacecraft has been inserted onto the halo orbit, a transfer onto the unstable invariant manifold will need to be completed in order to get on an interplanetary 
trajectory. Two methods were considered for how to use the manifolds to complete an interplanetary transfer. The first involves connecting the unstable manifold of a halo orbit at the departure planet with the stable manifold of the halo orbit at the arrival planet using patched conics. The second uses the unstable and stable manifolds to achieve lower energy transfers onto and off of hyperbolic escape trajectories at the departure and destination planets. Both methods will be described here, but in the end the second method ended up producing lower $\Delta v$ values and will be used for all test cases in later chapters.

\subsubsection{Patched Conics with Manifolds Method}

The idea for using patched conics between invariant manifolds is shown to be successful in Topputo et al. for transfers to Venus and Mars [16]. For this thesis, the goal is to complete transfers to Jupiter and Saturn, so the idea was adapted to those such trajectories. The method used here is to calculate arrival and departure $\Delta v$ values using Lambert's solution in the same way a normal patched conics trajectory would, but for points within the manifold instead of at the spheres of influence of the planets.

First, the unstable invariant manifolds for the halo orbit at the departure planet and the stable invariant manifolds for the halo orbit at the destination planet are calculated. The unstable manifold is required for departure since it is desired to leave the halo, while the stable manifold is required for the arrival planet as a spacecraft should approach the halo here. Then, points within these manifolds are tested as arrival and departure points for the interplanetary transfer. Narrowing down the actual locations within the manifolds to complete the transfer between proved to be very challenging since there are infinitely many possibilities. The method of using Poincare sections utilized by Ross [15] could be considered for future work, but it was deemed out of the scope of this thesis. Instead, points within the manifolds with 
high velocities were the first tested for possible arrival and departure locations. This method did not yield feasible $\Delta v$ values, and was discarded. Due to the problem of infinitely many departure and arrival points a new method proposed in Nakamiya et al. [8] was adopted.

\subsubsection{Manifold to Hyperbolic Escape Trajectory Method}

The new process for finding interplanetary transfer trajectories uses traditional patched conics for the transfers from planet to planet. That is, hyperbolic escape trajectories about each planet are connected using an elliptical transfer about the Sun. The difference is that rather than just maneuvering onto or off of the hyperbolic trajectories from or to orbits about the planets, manifolds can be used to reduce the $\Delta v$ for getting onto or off of the escape trajectories. A typical burn for getting onto an escape trajectory to a planet such as Jupiter or Saturn from a parking orbit about Earth is very large and often means a mission must complete multiple flybys of closer planets to reach its final destination. Reducing that $\Delta v$ could be very advantageous for certain missions that would be better off with an itinerary that does not require multiple gravity assists. Missions that need multiple gravity assists to reach their destinations must have spacecraft designed to survive more, and often harsher environments at the various planets along their journeys. If manifolds can be used to reduce $\Delta v$ and avoid these extra environmental considerations that could make them a viable solution for missions to distant planets.

Again, for this method the unstable invariant manifolds for the departure planet halo must be calculated. At the destination planet, an unstable or stable invariant manifold can be used to get off of the hyperbolic escape trajectory depending on where the mission will take the spacecraft after arrival. Later investigation revealed that the stable manifolds produced better $\Delta v$ numbers though, so the stable manifolds were 
used at arrival. Another option is to not not use a manifold at all and just complete a flyby maneuver to the next planet in the mission schedule.

\section{Manifold Transfer Arrival}

First, the procedure for a transfer onto a manifold at the destination planet will be explained. The invariant manifolds for the halo orbit at the departure planet and at the arrival planet are calculated. These remain the same in the synodic frame regardless of the location of the planets about the sun, so the same manifolds can be used no matter what the departure and arrival dates are. A set of departure and arrival dates are tested to compare the $\Delta v$ required and find the optimal time to leave and arrive with the least amount of fuel for the mission. In order to test all of these dates the positions and velocities of the first planet at all departure dates and of the second planet at all arrival dates are calculated using the planetary ephemeris model. Then Lambert's solution is used to find the velocity required for the transfer from the first planet to the second planet for all arrival and departure dates. This yields the required hyperbolic excess velocity $\left(v_{\infty}\right)$ required of the hyperbolic trajectories at departure and arrival.

Then, various values for the radius of perigee of the departure and arrival hyperbolic trajectories are tested to find the transfer with the least $\Delta v$. For every arrival and departure date multiple values for the magnitude of the radius of periapsis of the departure hyperbola $\left(r_{p}\right)$ and arrival hyperbola are tested with the manifold trajectories. The design of the hyperbolic escape trajectory at the departure planet can be seen in Figure 4.4 and can be found in Curtis [2]. 


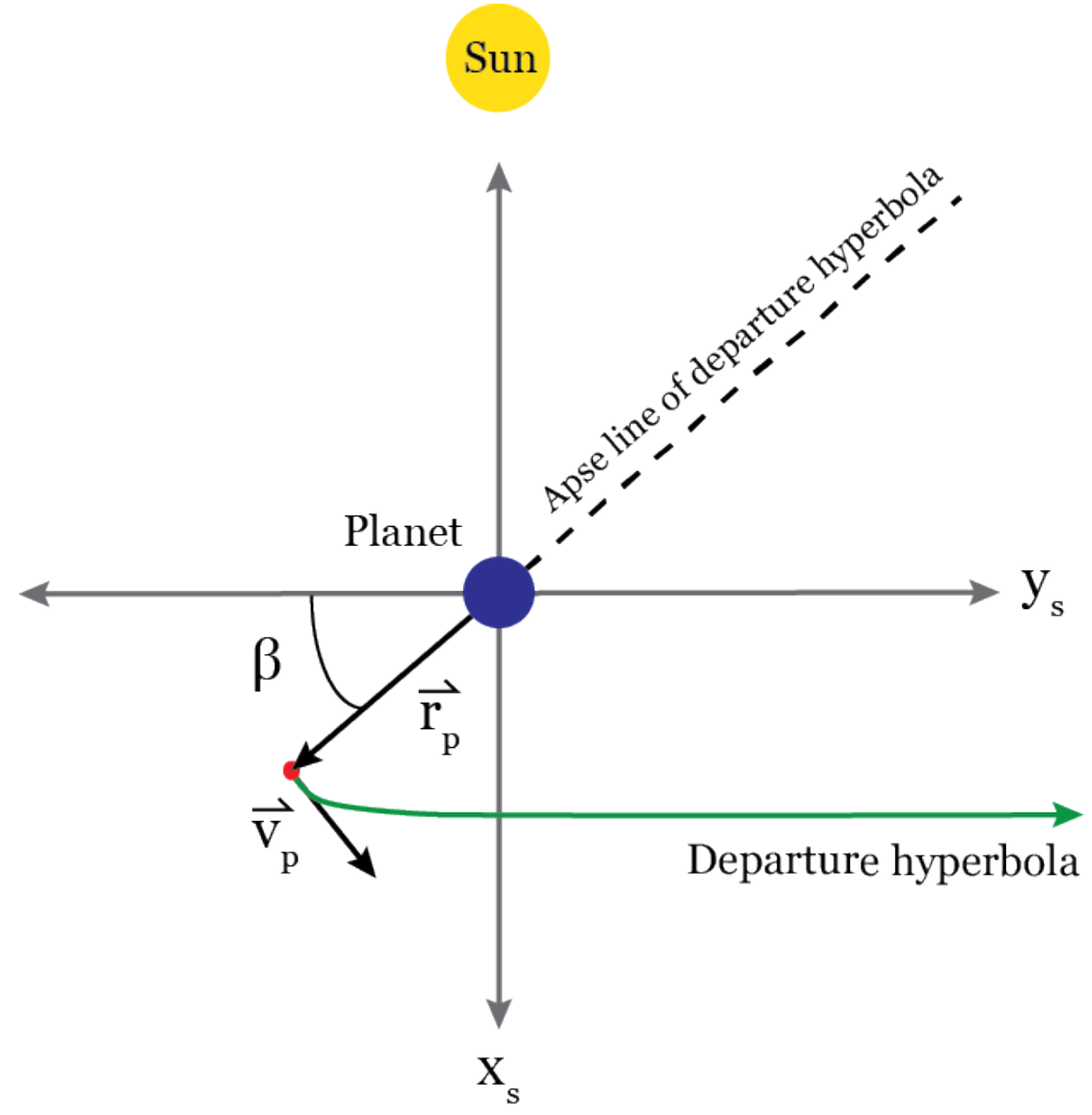

Figure 4.4: Hyperbolic escape trajectory geometry.

For this section of analysis the origin of the synodic frame has been translated so that the it is at the center of mass of the departure planet. The direction of the departure hyperbola is more advantageous for manifold trajectories coming from $\mathrm{L}_{1}$. The angle $\beta$ can be found using the following equation, where $e$ is the eccentricity of the hyperbolic trajectory [2]. The required eccentricity is based off of the hyperbolic escape velocity at Earth's sphere of influence $(924,540 \mathrm{~km})$ and the radius of periapsis of the hyperbola.

$$
\beta=\cos ^{-1}\left(\frac{1}{e}\right)
$$

For this set up of the hyperbolic departure, the position and velocity vectors at the radius of perigee of the hyperbola in the translated synodic frame are given by 
the following:

$$
\begin{aligned}
\vec{r}_{p} & =\left[\begin{array}{llll}
r_{p} \sin (\beta) & r_{p} \cos (\beta) & 0 &
\end{array}\right] \\
\vec{v}_{p} & =\left[\begin{array}{lllll}
v_{p} \cos (\beta) & -v_{p} \sin (\beta) & 0
\end{array}\right]
\end{aligned}
$$

For arrival at the destination planet the hyperbolic trajectory is slightly different, and can be seen in Figure 4.5.

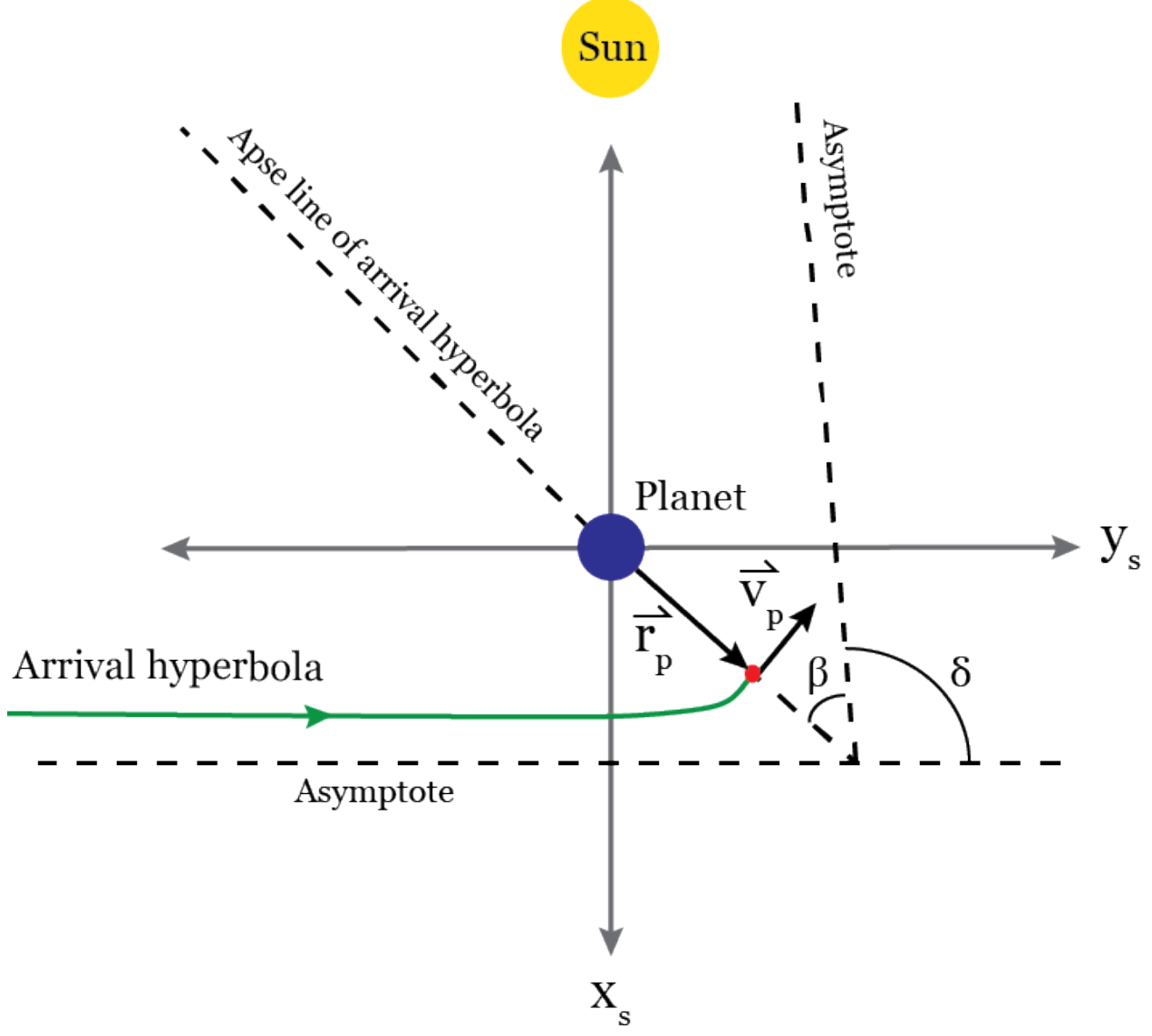

Figure 4.5: Hyperbolic arrival trajectory geometry.

Here $\beta$ can again be found using Equation 4.3 , and $\delta$ is computed using the following:

$$
\delta=2 \sin ^{-1}\left(\frac{1}{e}\right)
$$

The equations for the position and velocity vectors at the radius of periapsis of 
the arrival hyperbolic trajectory are shown in Equation 4.7 and Equation 4.8.

$$
\begin{gathered}
\vec{r}_{p}=\left[\begin{array}{lll}
r_{p} \sin \left(180^{\circ}-\delta-\beta\right) & r_{p} \cos \left(180^{\circ}-\delta-\beta\right) & 0
\end{array}\right] \\
\vec{v}_{p}=\left[\begin{array}{lll}
-v_{p} \cos \left(180^{\circ}-\delta-\beta\right) & v_{p} \sin \left(180^{\circ}-\delta-\beta\right) & 0
\end{array}\right]
\end{gathered}
$$

At both ends of the transfer, 500 trajectories within the invariant manifolds are tested to see which point in which trajectory passes the closest to the radius of periapsis of the hyperbolic escape trajectory. Once this point is found, the position and velocity at that point within the manifold trajectory are used to estimate the ideal inclination of the hyperbolic trajectory. Since the hyperbolic trajectory can be rotated about the apse line to any angle [2], the magnitude of this rotation (the inclination) can be altered to match the manifold trajectory. By taking the cross product of the position and velocity vectors of the closet approach point within the manifold, the specific angular momentum vector $(\vec{h})$ is acquired. Then inclination $(i)$ is found using the following equation.

$$
i=\cos ^{-1}\left(\frac{h_{z}}{h}\right)
$$

Then, the vectors of the hyperbolic trajectory at periapsis are rotated for this inclination change so that they become the following for escape hyperbolas:

$$
\begin{gathered}
\vec{r}_{h y p}=\left[\begin{array}{lll}
r_{p} \sin (\beta) \cos (i) & -r_{p} \cos (\beta) & r_{p} \sin (\beta) \sin (i)
\end{array}\right] \\
\vec{v}_{h y p}=\left[\begin{array}{lll}
v_{p} \cos (\beta) \cos (i) & v_{p} \sin (\beta) & v_{p} \cos (\beta) \sin (i)
\end{array}\right]
\end{gathered}
$$

and the following for arrival hyperbolas:

$$
\begin{aligned}
& \vec{r}_{h y p}=\left[\begin{array}{lll}
r_{p} \sin \left(180^{\circ}-\delta-\beta\right) \cos (i) & r_{p} \cos \left(180^{\circ}-\delta-\beta\right) & -r_{p} \sin \left(180^{\circ}-\delta-\beta\right) \sin (i)
\end{array}\right] \\
& \vec{v}_{h y p}=\left[\begin{array}{lll}
-v_{p} \cos \left(180^{\circ}-\delta-\beta\right) \cos (i) & v_{p} \sin \left(180^{\circ}-\delta-\beta\right) & -v_{p} \cos \left(180^{\circ}-\delta-\beta\right) \sin (i)
\end{array}\right]
\end{aligned}
$$


These results are then used to calculate the $\Delta v$ required for departure and arrival on the invariant manifolds by finding the difference in the velocity vectors.

$$
\begin{aligned}
& \Delta v_{d}=\left|\vec{v}_{h y p, d}-\vec{v}_{m a n, d}\right| \\
& \Delta v_{a}=\left|\vec{v}_{m a n, a}-\vec{v}_{h y p, a}\right|
\end{aligned}
$$

The subscripts on the velocity vectors designate whether the velocity is on the manifold or the hyperbolic trajectory.

An example for the departure maneuver can be seen in Figure 4.6. The purple trajectory is one within the manifold, while the green is the hyperbolic escape trajectory. The red star is the perigee point of the hyperbola and the yellow star is the departure point from the manifold. Although they do not line up perfectly it is assumed that this error can be corrected for with further orbital analysis. The work required for that is out of the scope of this thesis. The zoomed out view that includes the entire manifold trajectory is shown in Figure 4.7. 


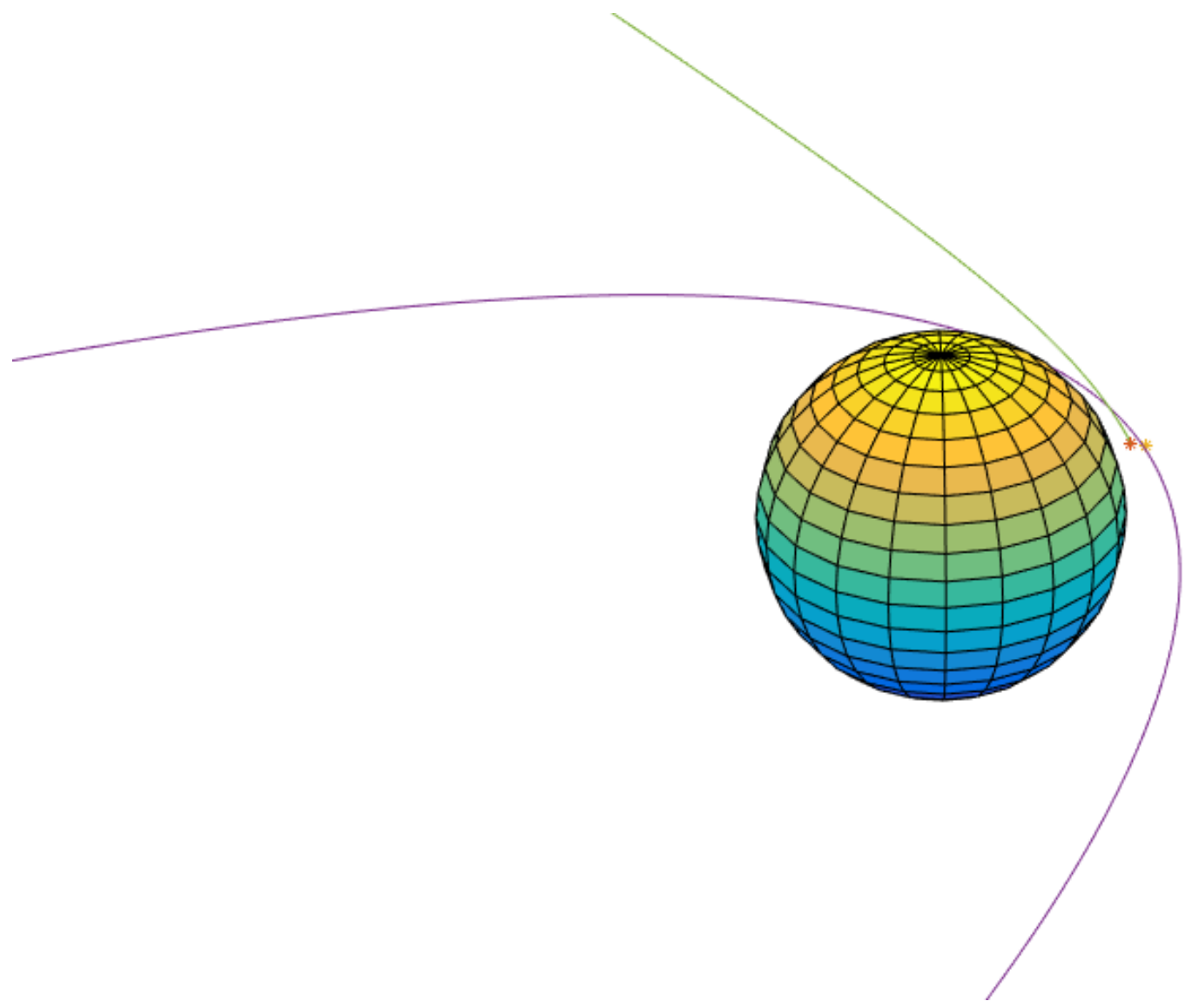

Figure 4.6: Example of intersection point between the trajectory within the unstable invariant manifold (purple) and the hyperbolic escape trajectory (green) to leave Earth. 


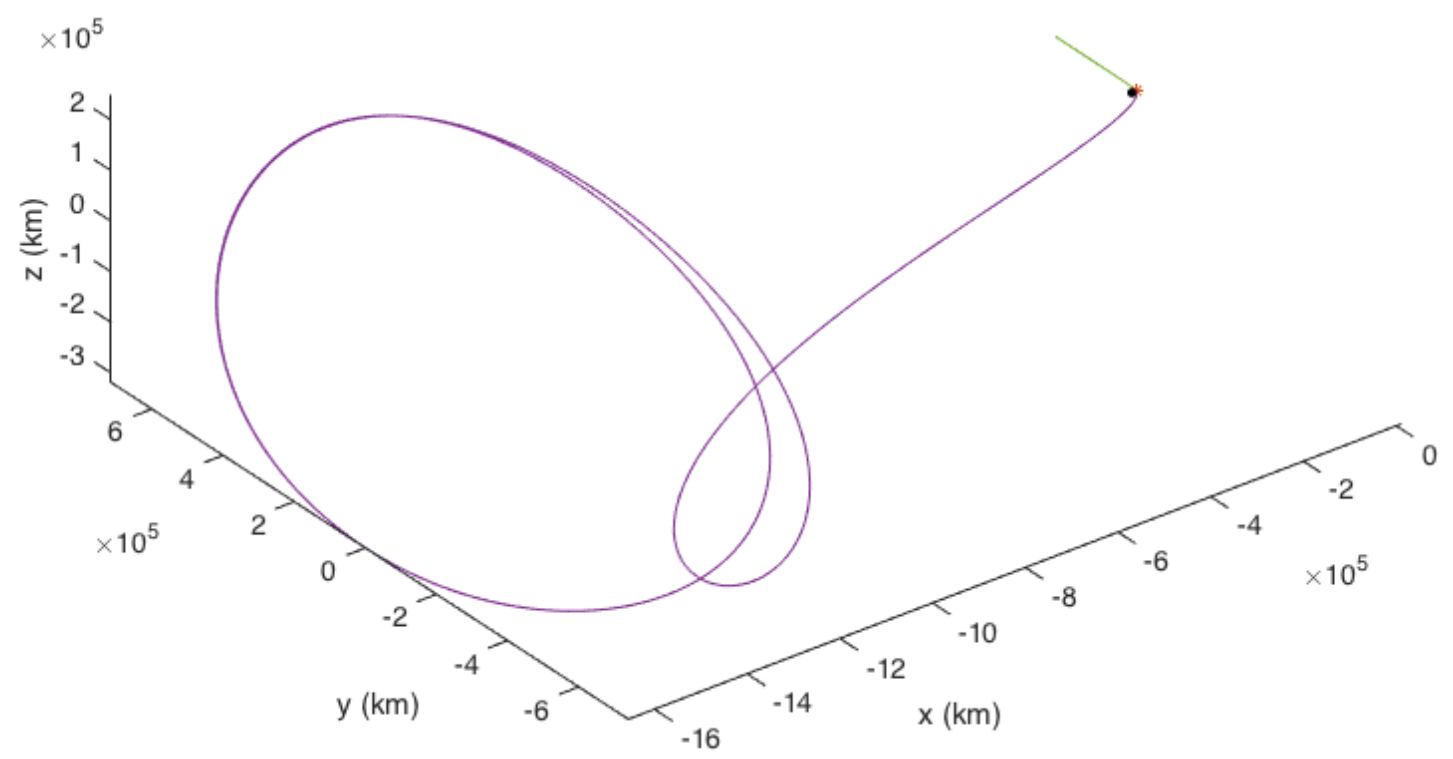

Figure 4.7: Earth departure example showing full manifold trajectory.

An arrival trajectory at Jupiter can be observed in Figure 4.8. Again, the manifold trajectory can be seen in purple, and the incoming hyperbolic trajectory in green. The star point shows the transfer location. The full manifold trajectory from the halo orbit is shown in Figure 4.9.

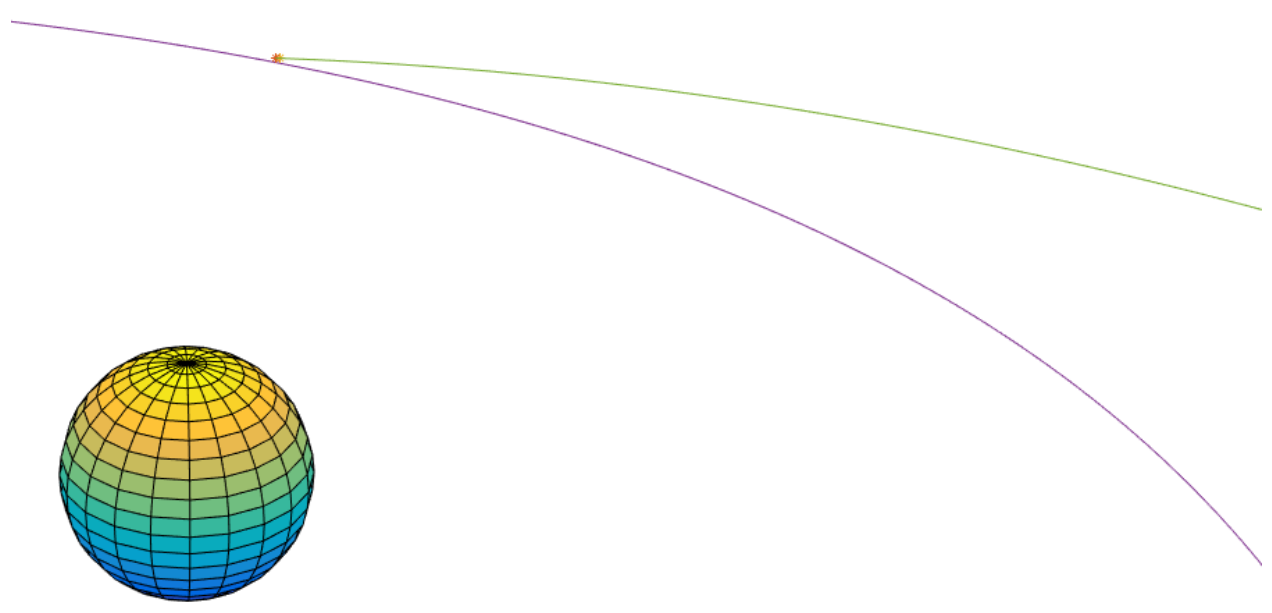

Figure 4.8: Example of transfer point from incoming hyperbolic trajectory onto a trajectory within a manifold at Jupiter. 


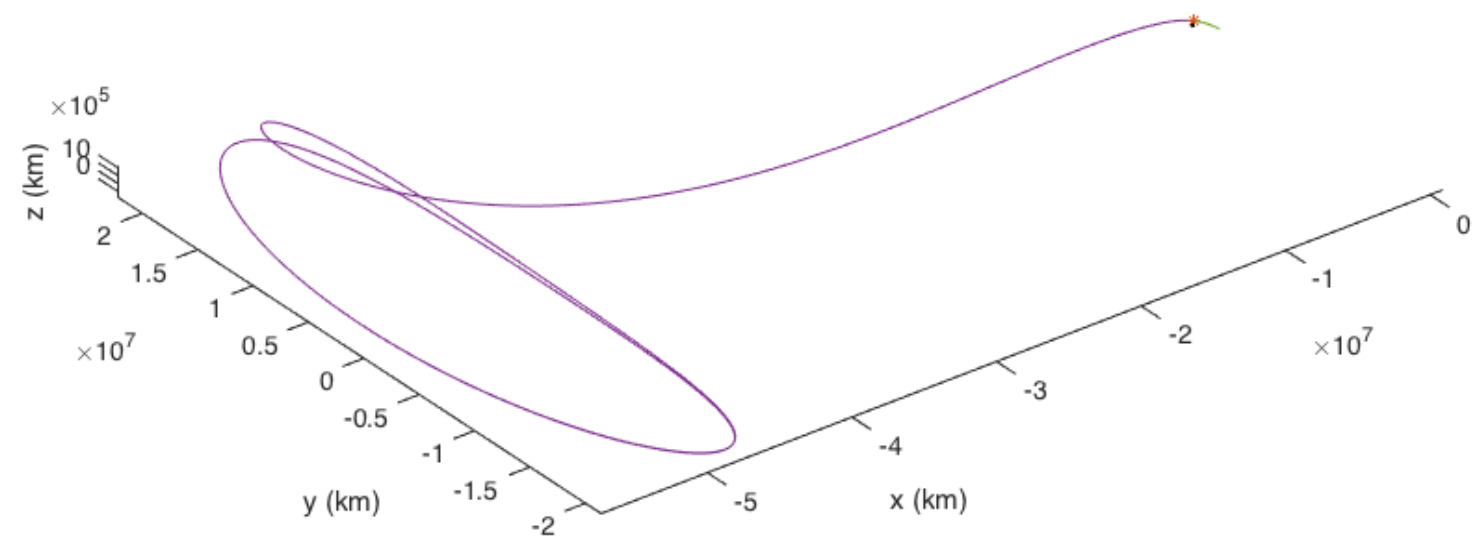

Figure 4.9: Jupiter arrival example showing full manifold trajectory.

The Matlab code that was written to perform this analysis was verified with various sources in order to ensure that all positions and velocities within trajectories are correctly calculated. The invariant manifolds and halo orbits match results found in Koon et al. [7], and the hyperbolic trajectory calculation was checked with examples in Curtis [2].

\section{Flyby Maneuver Arrival}

If a mission required a flyby at the destination planet, the usual steps required to calculate the dynamics of a gravity assist would be employed. Departure and arrival date options would be much more limited as the $v_{\infty}$ arriving at the original destination planet would need to match that required to transfer on the next planet. Using manifolds in the method presented here would then only be needed at departure from Earth. The actual flyby trajectory would use traditional patched conics methods. The full analysis will not be outlined here, but a gravity assist is certainly possible in this mission design plan. 
Chapter 5

\section{MISSION APPLICATIONS AND RESULTS}

\subsection{Overview}

This method of completing interplanetary transfers has the potential to reduce the total $\Delta v$ required for missions to distant planets. The transfer from a trajectory within a manifold onto a hyperbolic escape trajectory requires significantly less $\Delta v$ than a transfer from a parking orbit. At arrival planets, the transfer off of a hyperbolic trajectory onto a manifold trajectory also costs less than a transfer onto an orbit about the planet. A spacecraft on a manifold at a destination planet could then continue to use the CRTBP to travel about that system. This section will cover various test cases that were run in order to find out if this method of interplanetary transfers could reduce overall mission $\Delta v$. Missions to Jupiter and Saturn will be investigated, and the $\Delta v$ found will be compared to that required for a normal patched conics transfers. The time of flight required, and other mission considerations will also be discussed.

\subsection{Earth to Jupiter Transfer}

Since so many scientific missions to outer planets are interested in either investigating Jupiter itself or using it to complete a flyby maneuver, the first transfer case tested was from Earth to Jupiter. The main goal was to find a method of patching together the various components of the trajectories in such a way that the $\Delta v$ could be reduced from that of a typical patched conics trajectory from Earth to Jupiter. If using manifolds can significantly reduce overall required $\Delta v$ to get to Jupiter, it could end up being more useful than using multiple flybys of inner planets. Often those flybys 
induce greater thermal and environmental requirements of a spacecraft since it has to go closer to the sun and encounter harsh atmospheres (especially for Venus flybys).

A transfer leaving Earth and arriving at Jupiter around the dates of Voyager 1 was investigated. Dates around the actual departure and arrival dates for the Voyager spacecraft (September 5, 1977 and March 5, 1979) were tested for various sizes of halo orbits at Earth and at Jupiter. After initial tests it was found that using the unstable invariant manifolds of a halo orbit about Sun-Earth $\mathrm{L}_{1}$ was optimal for transferring onto a hyperbolic escape trajectory from Earth. This was also found for cases of transfers to Mars in Nakamiya et al. [8]. Due to this finding, all the test cases reported here are run with halo orbits about Sun-Earth $\mathrm{L}_{1}$ at the departure end of the transfer. After preliminary tests it was also found that using the stable manifold of a halo about Sun-Jupiter $\mathrm{L}_{1}$ lined up the best with the arrival hyperbolic trajectories. This means all arrival cases are also tested for halos about $\mathrm{L}_{1}$ at Jupiter.

First wide ranges of halo orbit sizes were tested to see which yielded lower $\Delta v$ values. It was found that halo orbits with very large or very small $A_{z}$ amplitudes tended to yield higher amounts of $\Delta v$ for the maneuver onto or off of the hyperbolic escape trajectories at departure and arrival. It was also discovered that southern halos for departure from Earth, and northern halos for arrival at Jupiter produced much lower $\Delta v$ values. Therefore, the results shown here are for halo orbits tested in the realm where the lowest $\Delta v$ values were found, and where the hyperbolic trajectories lined up with the invariant manifolds. The reason these halo orientations yielded lower $\Delta v$ values may have to do with the directions of the manifolds and the orbits of the planets, but further investigation could yield a more definitive answer. The steps outlined in chapter 4 are used to find the $\Delta v$ values for all the different dates of departure and arrival. The optimal transfer dates from the range inputted are found based on the lowest total $\Delta v$ required for departure and arrival maneuvers. The different $\Delta v$ values found for various sizes of departure and arrival halos can be 
seen in Table 5.1. Here, the departure $\Delta v$ is for the maneuver from the manifold onto the hyperbolic escape trajectory at Earth, and the arrival $\Delta v$ is for the maneuver from the hyperbolic trajectory onto the manifold at Jupiter.

Table 5.1: $\Delta v$ values for manifold-hyperbolic transfer for various sized halos at Earth and Jupiter.

\begin{tabular}{ccccc} 
Earth Halo $\mathrm{A}_{z}$ & Jupiter Halo $\mathrm{A}_{\mathrm{z}}$ & Departure $\Delta v$ & Arrival $\Delta v$ & Total $\Delta v$ \\
\hline $250,000 \mathrm{~km}$ & $1,000,000 \mathrm{~km}$ & $4.70 \mathrm{~km} / \mathrm{s}$ & $7.13 \mathrm{~km} / \mathrm{s}$ & $11.83 \mathrm{~km} / \mathrm{s}$ \\
$310,000 \mathrm{~km}$ & $950,000 \mathrm{~km}$ & $4.76 \mathrm{~km} / \mathrm{s}$ & $7.03 \mathrm{~km} / \mathrm{s}$ & $11.80 \mathrm{~km} / \mathrm{s}$ \\
$280,000 \mathrm{~km}$ & $800,000 \mathrm{~km}$ & $4.66 \mathrm{~km} / \mathrm{s}$ & $7.08 \mathrm{~km} / \mathrm{s}$ & $11.74 \mathrm{~km} / \mathrm{s}$ \\
$280,000 \mathrm{~km}$ & $1,000,000 \mathrm{~km}$ & $4.64 \mathrm{~km} / \mathrm{s}$ & $7.04 \mathrm{~km} / \mathrm{s}$ & $11.69 \mathrm{~km} / \mathrm{s}$ \\
$280,000 \mathrm{~km}$ & $900,000 \mathrm{~km}$ & $4.65 \mathrm{~km} / \mathrm{s}$ & $7.02 \mathrm{~km} / \mathrm{s}$ & $11.67 \mathrm{~km} / \mathrm{s}$
\end{tabular}

As can be seen in the table above the lowest total $\Delta v$ found for these dates was $11.67 \mathrm{~km} / \mathrm{s}$. For this transfer the Earth departure date is September 2, 1977 and the Jupiter arrival date is March 9, 1979. The trajectory from Earth to Jupiter for the given dates can be seen in Figure 5.1. 


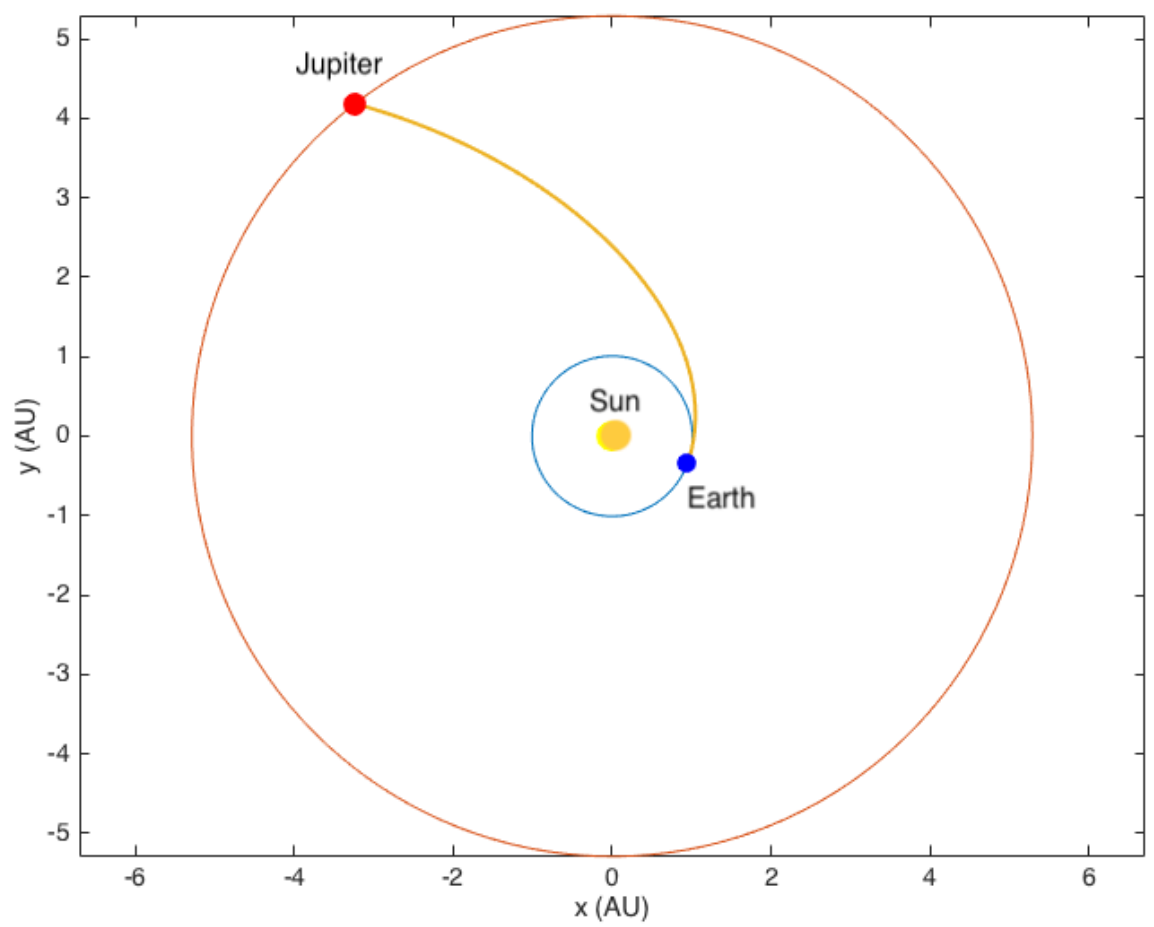

Figure 5.1: Transfer trajectory about the sun from Earth to Jupiter, showing Earth's position at departure and Jupiter's position at arrival.

The transfer off of the unstable invariant manifold onto the hyperbolic escape trajectory at Earth is shown in Figure 5.2 and Figure 5.3. The halo orbit size producing the lowest $\Delta v$ is $280,000 \mathrm{~km} \mathrm{z}$-amplitude, and the altitude of periapsis of the departure hyperbola is $651.7 \mathrm{~km}$. The required $\Delta v$ for this maneuver is $4.65 \mathrm{~km} / \mathrm{s}$. There is an error of $-418.6 \hat{x}+249.6 \hat{y}+127.9 \hat{z} \mathrm{~km}$ in the positioning of the departure point on the manifold and the radius of periapsis of the hyperbolic trajectory, as can be seen in Figure 5.3. This error is relatively small and it is assumed that with orbital corrections and more in-depth analysis this error could be significantly reduced. For the scope of this thesis, the error was decided to be acceptable. 

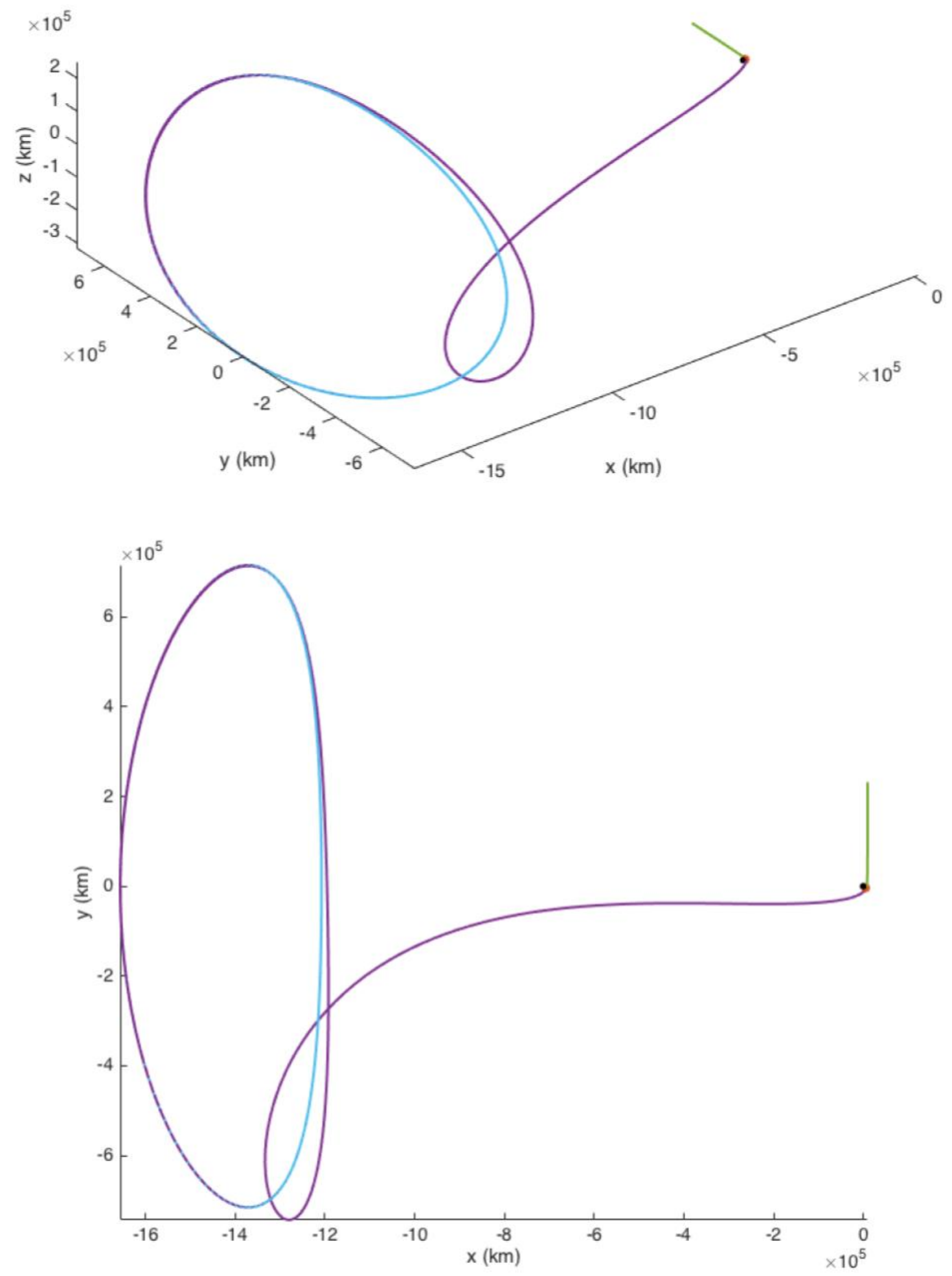

Figure 5.2: Full view of transfer from halo orbit (blue) to trajectory within manifold (purple) to hyperbolic escape trajectory (green). 

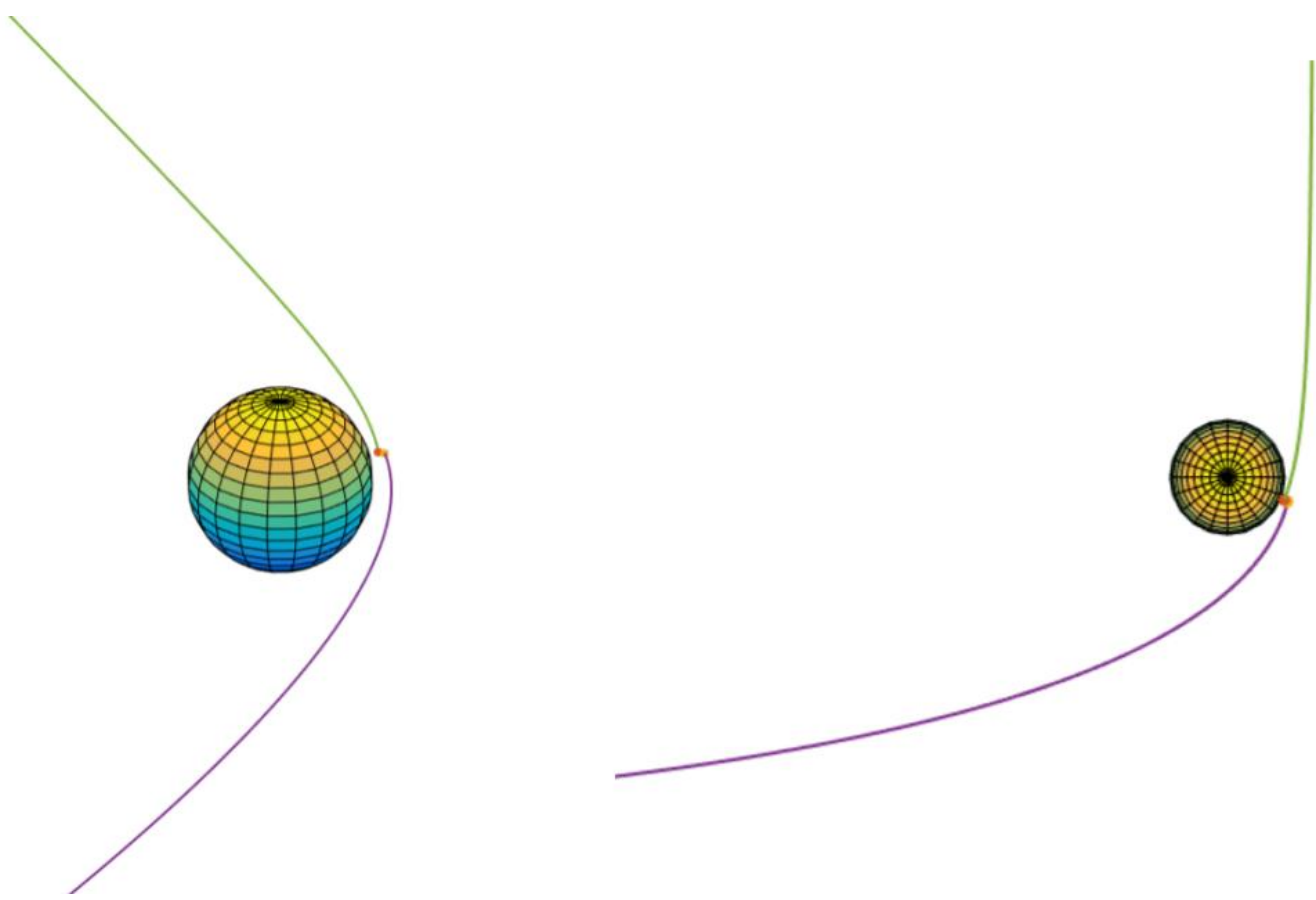

Figure 5.3: Close up views of transfer from manifold (purple) onto hyperbolic escape trajectory (green) at Earth. The yellow star shows the departure point on the manifold and the red star shows the injection point on the hyperbola.

The maneuver from the hyperbolic trajectory onto the stable invariant manifold at Jupiter can be seen in Figure 5.4 and Figure 5.5. The halo orbit size for this transfer is $900,000 \mathrm{~km}$ in $\mathrm{z}$-amplitude and the altitude of periapsis of the hyperbola is 403,670 $\mathrm{km}$. The $\Delta v$ required is $7.02 \mathrm{~km} / \mathrm{s}$, bringing the total $\Delta v$ for the interplanetary transfer to $11.67 \mathrm{~km} / \mathrm{s}$. 

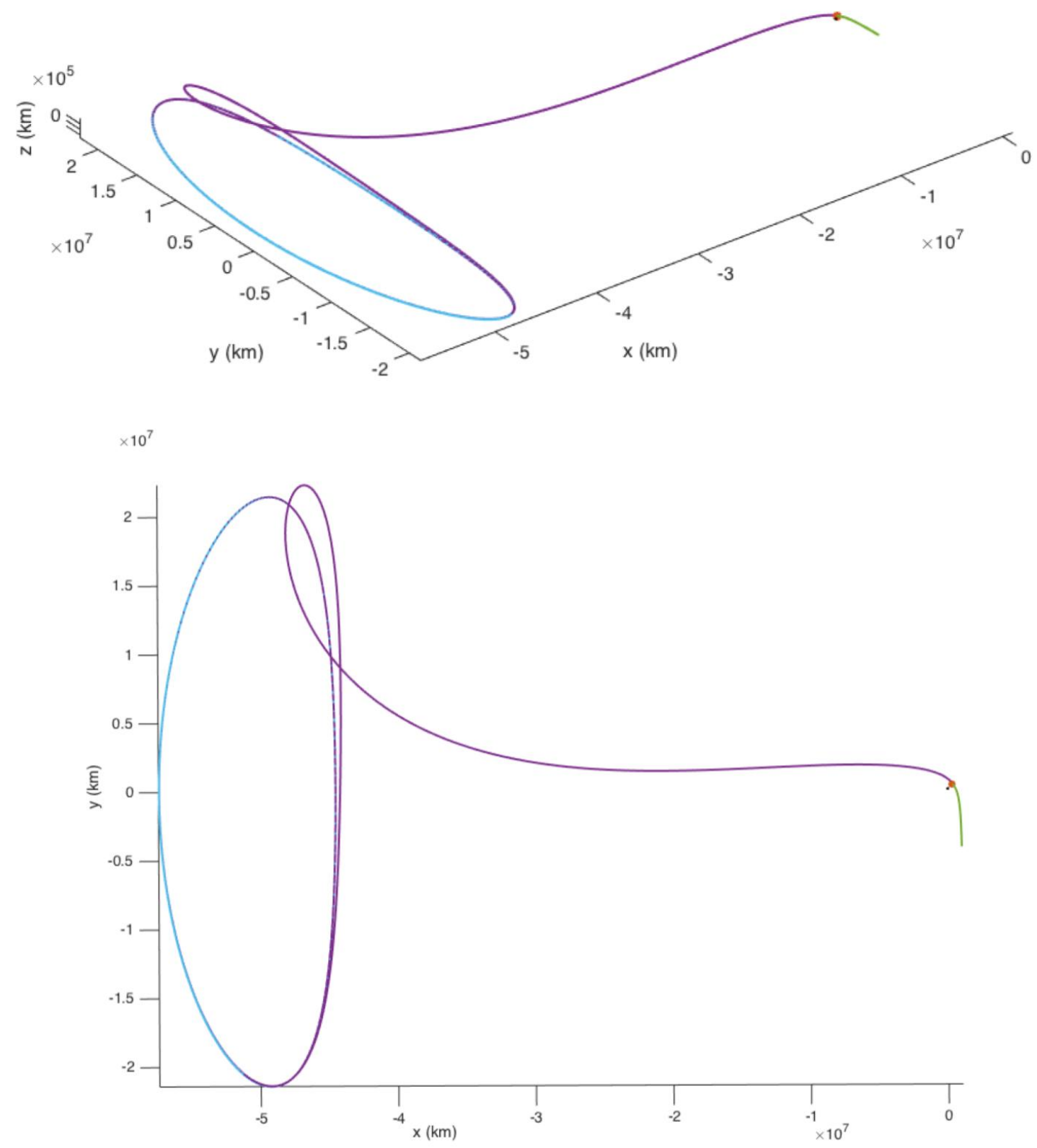

Figure 5.4: Full view of transfer from hyperbolic escape trajectory (green) to trajectory within manifold (purple) to halo orbit (blue). 


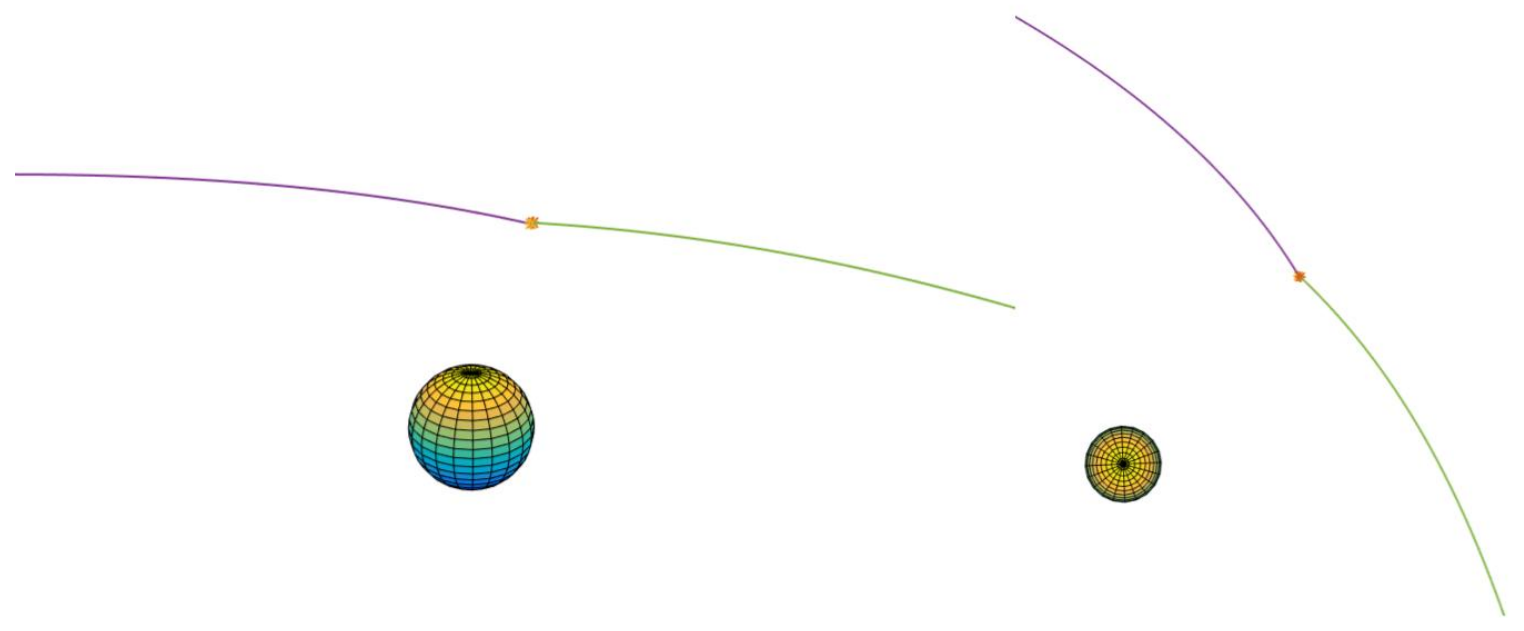

Figure 5.5: Close up views of transfer from hyperbolic escape trajectory (green) to manifold (purple) upon arrival at Jupiter. The yellow star shows the injection point on the manifold and the red star shows the departure point on the hyperbola.

These $\Delta v$ values are indeed high, but are much less than the $\Delta v$ required for a traditional patched conics trajectory, where a burns from and to orbits about the planets would need to be completed. A comparison of the lowest computed total $\Delta v$ for the manifold method can be compared to the $\Delta v$ required for transfers from circular orbits at the same periapsis altitudes about Earth and Jupiter to and from the hyperbolic escape trajectories can be seen in Table 5.2.

Table 5.2: Comparison of the $\Delta v$ required for traditional patched conics maneuvers and for the manifold maneuvers.

\begin{tabular}{c|ccc} 
Transfer Type & Departure $\Delta v$ & Arrival $\Delta v$ & Total $\Delta v$ \\
\hline Manifold & $4.65 \mathrm{~km} / \mathrm{s}$ & $7.02 \mathrm{~km} / \mathrm{s}$ & $11.67 \mathrm{~km} / \mathrm{s}$ \\
Traditional & $7.30 \mathrm{~km} / \mathrm{s}$ & $8.50 \mathrm{~km} / \mathrm{s}$ & $15.80 \mathrm{~km} / \mathrm{s}$
\end{tabular}

It is clear from this comparison that using the manifold transfers can significantly reduce the $\Delta v$ at both ends of the interplanetary transfer. At Earth the $\Delta v$ is reduced by $2.64 \mathrm{~km} / \mathrm{s}$ and at Jupiter by $1.48 \mathrm{~km} / \mathrm{s}$, leading to a reduced total $\Delta v$ of $4.12 \mathrm{~km} / \mathrm{s}$ 
for the interplanetary transfer. It is important to note that for the manifold transfer the $\Delta v$ to get to the halo orbit in the first place needs to be included in the overall mission $\Delta v$. The $\Delta v$ required to get to the Earth halo from a LEO parking orbit is $3.09 \mathrm{~km} / \mathrm{s}$, bringing the total $\Delta v$ to get from Earth to Jupiter to $14.76 \mathrm{~km} / \mathrm{s}$. The total $\Delta v$ and time of flight $(\mathrm{ToF})$ comparison for a traditional transfer and all the components of the manifold transfer can be seen in Table 5.3. The time of flight for the manifold method is longer because of the need to get to the halo orbit and back.

Table 5.3: Comparison of the total $\Delta v$ required for mission and the time of flight (ToF) for traditional and manifold methods.

\begin{tabular}{c|cccc} 
Transfer Type & Halo Insertion $\Delta v$ & Transfer $\Delta v$ & Total $\Delta v$ & ToF \\
\hline Manifold & $3.09 \mathrm{~km} / \mathrm{s}$ & $11.67 \mathrm{~km} / \mathrm{s}$ & $14.76 \mathrm{~km} / \mathrm{s}$ & 919 days \\
Traditional & $0 \mathrm{~km} / \mathrm{s}$ & $15.80 \mathrm{~km} / \mathrm{s}$ & $15.80 \mathrm{~km} / \mathrm{s}$ & 553 days
\end{tabular}

As these results show, the overall mission $\Delta v$ is reduced by about $1 \mathrm{~km} / \mathrm{s}$. Although the $\Delta v$ for the maneuver onto the hyperbolic trajectory at Earth was greatly reduced by using the manifold, the $3 \mathrm{~km} / \mathrm{s}$ needed to get onto the manifold in the first place increases the $\Delta v$ to leave Earth to be slightly greater than that of a traditional transfer. Upon arrival at Jupiter there are still significant savings, and the dynamics of the CRTBP could be used to explore the Jovian system after arrival.

\subsection{Earth to Saturn Transfer}

Saturn is also a destination planet of great interest for scientific missions. More explorations of the planet itself, as well as its many moons are very likely to take place in the future, and the next test cases will see if a trajectory to Saturn is feasible using manifolds to assist in departure from Earth and arrival at Saturn. All missions that have flown to Saturn in the past have required a flyby of Jupiter, so this study 
will see if it is possible to get there without the Jupiter flyby. Again, reducing the number of flybys required to get the destination could help reduce the requirements on a spacecraft for surviving in different planets' harsh environments and simplify the trajectory.

Dates for a future mission to Saturn (leaving Earth around 2020) were tested, and departure and arrival times corresponding to the most energy efficient transfer based on the positions of the planets were used. These dates end up being mid-April 2020 for departure from Earth, and mid-May 2026 for arrival at Saturn. Various days around these times were tested for arrival and departure in order to find a better transfer $\Delta v$. As with the Jupiter test, it was found that southern halos at Earth, and northern halos at Saturn produced better results, so those are the only cases included here. Additionally, preliminary tests were run with much larger and smaller halo orbits at both planets, but these yielded much higher values for $\Delta v$, and so are not included here. The same process was completed, as with the Jupiter cases, to find the lowest total $\Delta v$, and the results for various sizes of halo orbits can be seen in Table 5.4 .

Table 5.4: $\Delta v$ values for manifold-hyperbolic transfer for various sized halos at Earth and Saturn.

\begin{tabular}{ccccc} 
Earth Halo $\mathrm{A}_{\mathrm{z}}$ & Saturn Halo $\mathrm{A}_{\mathrm{z}}$ & Departure $\Delta v$ & Arrival $\Delta v$ & Total $\Delta v$ \\
\hline $320,000 \mathrm{~km}$ & $700,000 \mathrm{~km}$ & $6.02 \mathrm{~km} / \mathrm{s}$ & $1.90 \mathrm{~km} / \mathrm{s}$ & $7.92 \mathrm{~km} / \mathrm{s}$ \\
$280,000 \mathrm{~km}$ & $600,000 \mathrm{~km}$ & $5.80 \mathrm{~km} / \mathrm{s}$ & $1.98 \mathrm{~km} / \mathrm{s}$ & $7.78 \mathrm{~km} / \mathrm{s}$ \\
$300,000 \mathrm{~km}$ & $800,000 \mathrm{~km}$ & $5.74 \mathrm{~km} / \mathrm{s}$ & $1.90 \mathrm{~km} / \mathrm{s}$ & $7.63 \mathrm{~km} / \mathrm{s}$ \\
$260,000 \mathrm{~km}$ & $700,000 \mathrm{~km}$ & $5.86 \mathrm{~km} / \mathrm{s}$ & $1.76 \mathrm{~km} / \mathrm{s}$ & $7.62 \mathrm{~km} / \mathrm{s}$ \\
$300,000 \mathrm{~km}$ & $700,000 \mathrm{~km}$ & $5.74 \mathrm{~km} / \mathrm{s}$ & $1.76 \mathrm{~km} / \mathrm{s}$ & $7.49 \mathrm{~km} / \mathrm{s}$
\end{tabular}

As the table above shows, the lowest total $\Delta v$ found for these dates was 7.49 $\mathrm{km} / \mathrm{s}$. For this transfer the Earth departure date is April 11, 2020 and the Saturn 
arrival date is May 9, 2026. The trajectory from Earth to Saturn for the given dates can be seen in Figure 5.6.

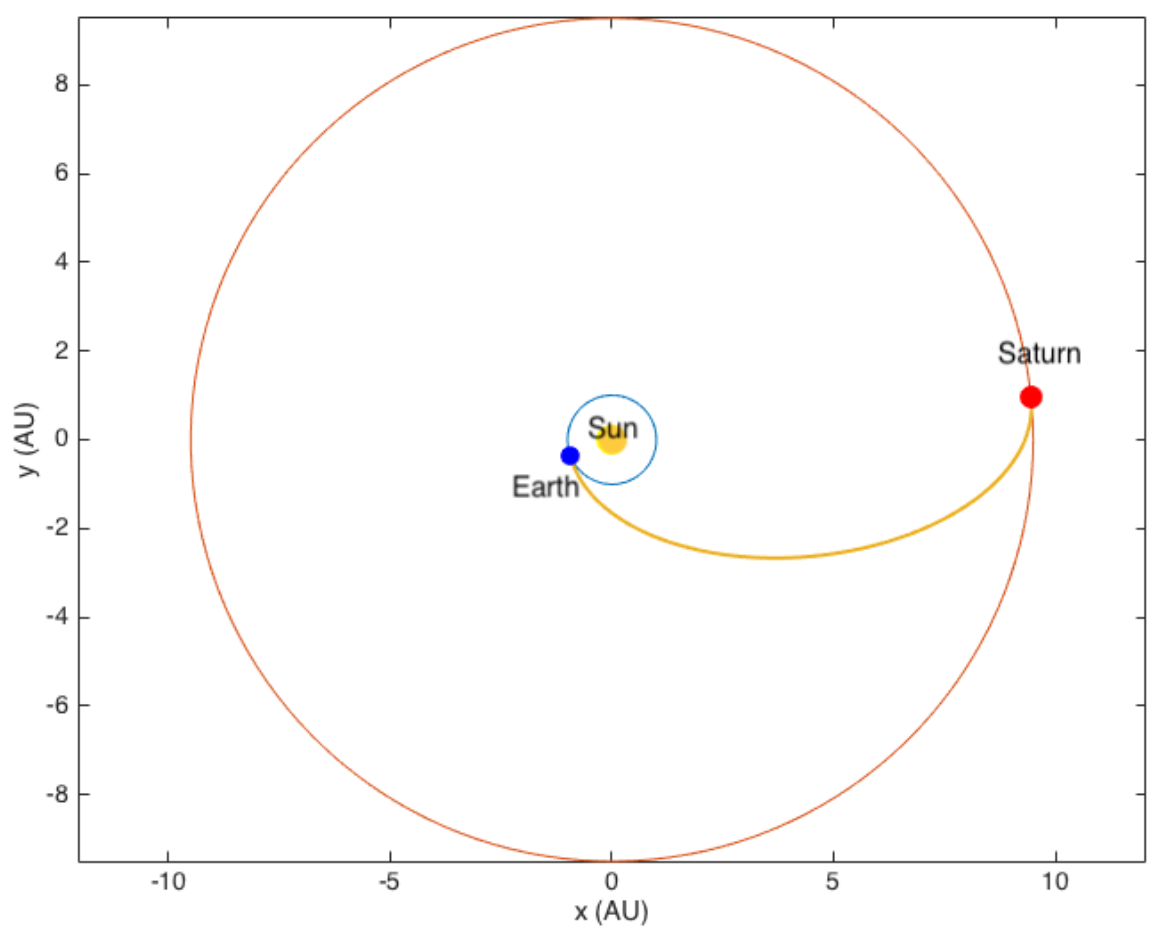

Figure 5.6: Transfer from Earth to Saturn about the sun, showing Earth at the time of departure and Saturn at the time of arrival.

The transfer off of the unstable invariant manifold onto the hyperbolic escape trajectory at Earth is shown in Figure 5.7 and Figure 5.8. The halo orbit size producing the lowest $\Delta v$ is $300,000 \mathrm{~km}$ in z-amplitude, and the altitude of periapsis of the departure hyperbola is $200.4 \mathrm{~km}$. This low altitude could cause some problems with drag in Earth's atmosphere, but further investigation would be necessary to see if there is any significant change in the trajectory. If drag was a problem, the periapsis altitude could have lower limits specified so that no solutions would pass too close to Earth. The required $\Delta v$ for this maneuver is $5.74 \mathrm{~km} / \mathrm{s}$. There is an error of $-218.3 \hat{x}+238.2 \hat{y}+182.3 \hat{z} \mathrm{~km}$ in the positioning of the departure point on 
the manifold and the radius of periapsis of the hyperbolic trajectory, as is shown in Figure 5.8. As with the Jupiter case, it was decided that for the scope of this thesis, the error was acceptable, but could reduced with further investigation.
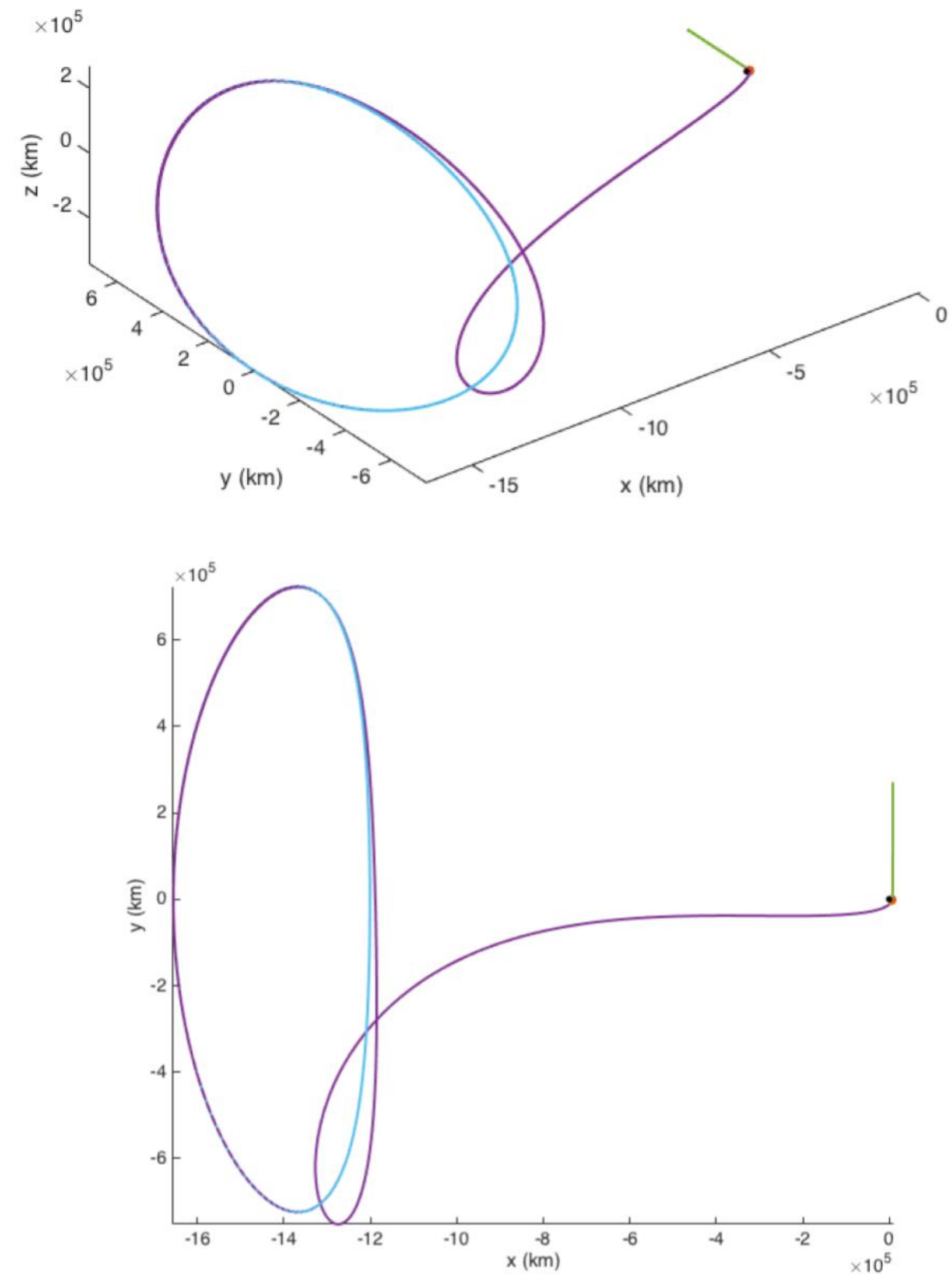

Figure 5.7: Full view of transfer from halo orbit (blue) to trajectory within manifold (purple) to hyperbolic escape trajectory (green). 

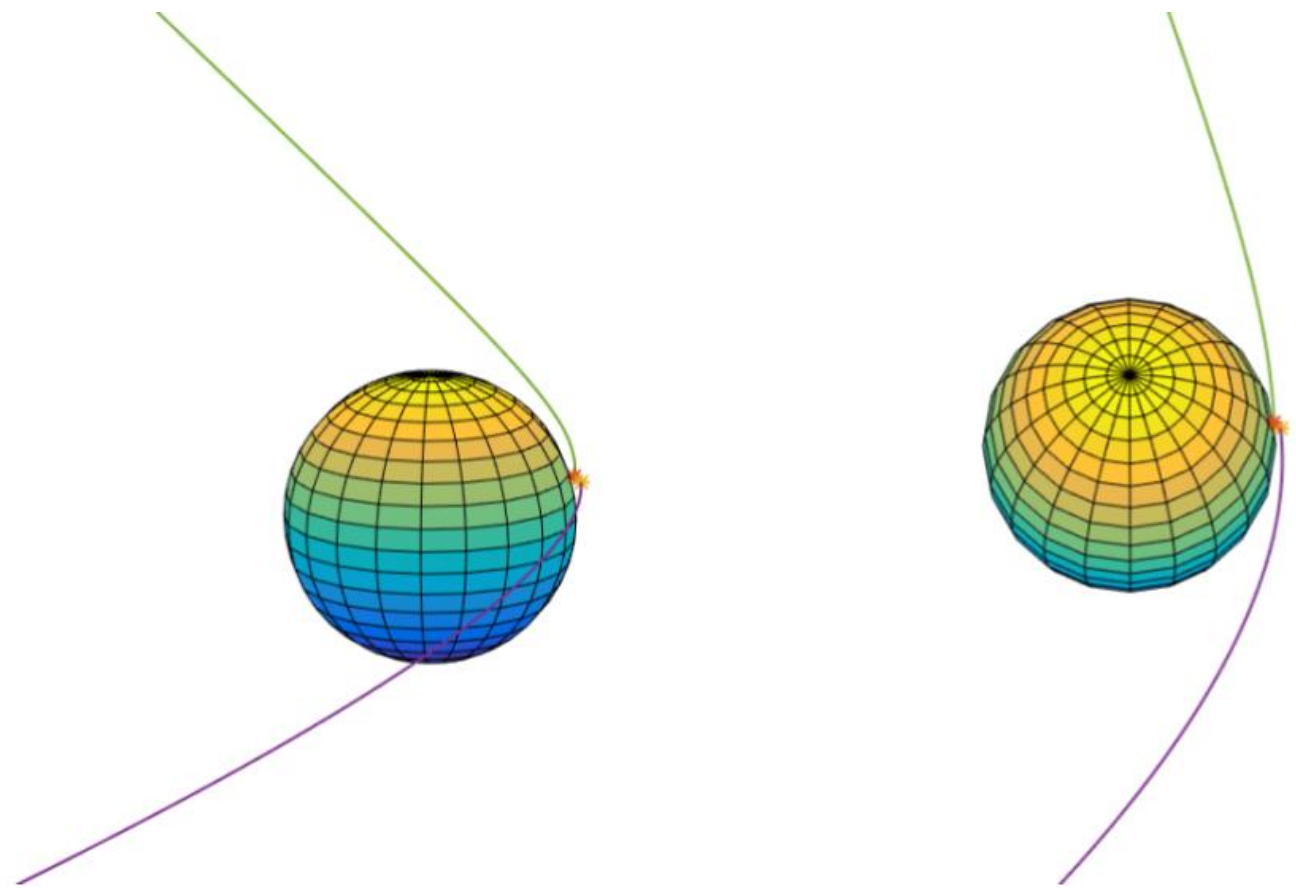

Figure 5.8: Close up views of transfer from manifold (purple) onto hyperbolic escape trajectory (green) at Earth. The yellow star shows the departure point on the manifold and the red star shows the injection point on the hyperbola.

The maneuver from the hyperbolic trajectory onto the stable invariant manifold at Saturn is shown in Figure 5.9 and Figure 5.10. The halo orbit size for this transfer is $700,000 \mathrm{~km}$ in $\mathrm{z}$-amplitude and the altitude of periapsis of the hyperbola is 788,464 $\mathrm{km}$. The $\Delta v$ required is $1.76 \mathrm{~km} / \mathrm{s}$, bringing the total $\Delta v$ for the interplanetary transfer to $7.49 \mathrm{~km} / \mathrm{s}$. 

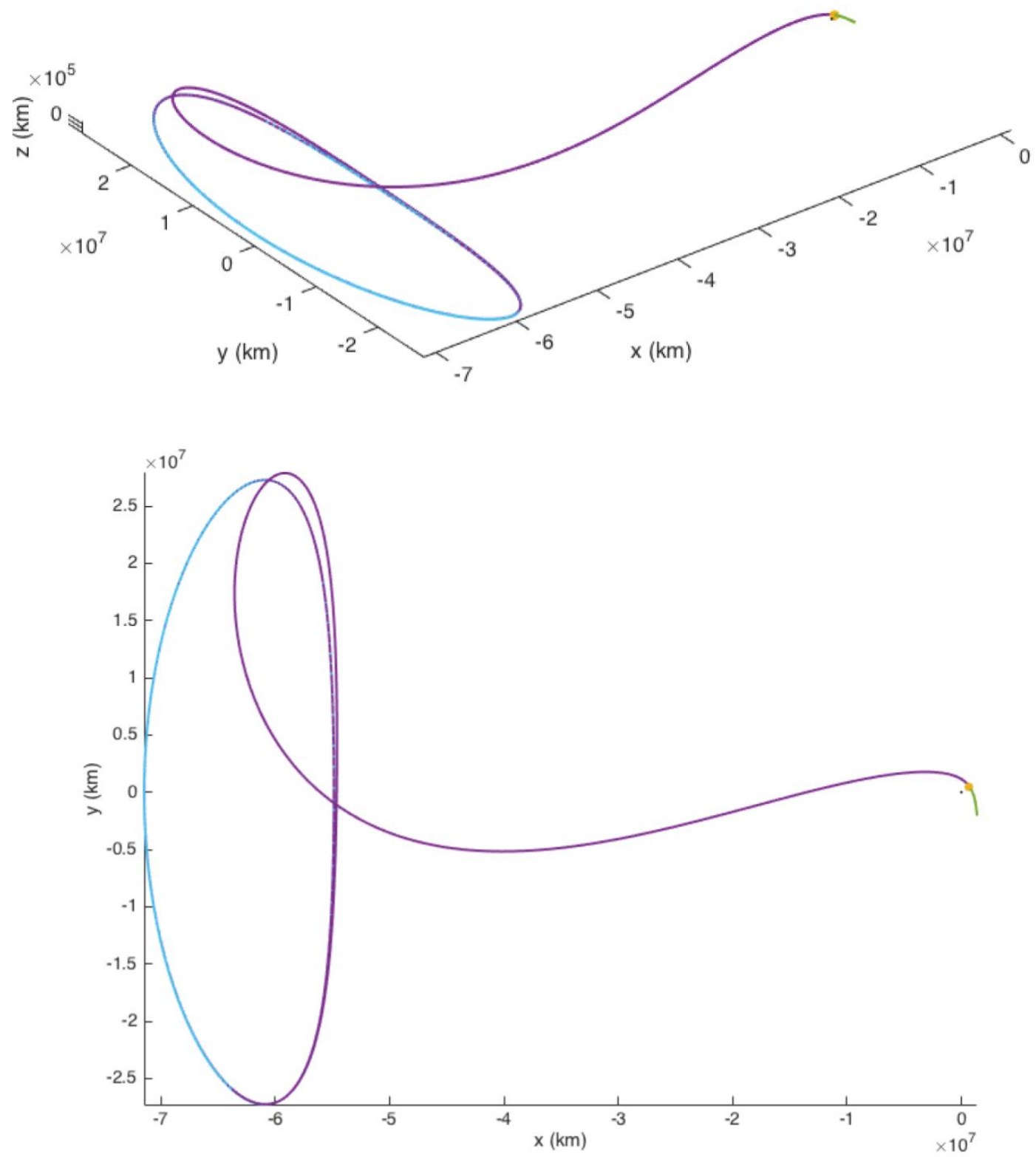

Figure 5.9: Full view of transfer from hyperbolic escape trajectory (green) to trajectory within manifold (purple) to halo orbit (blue). 


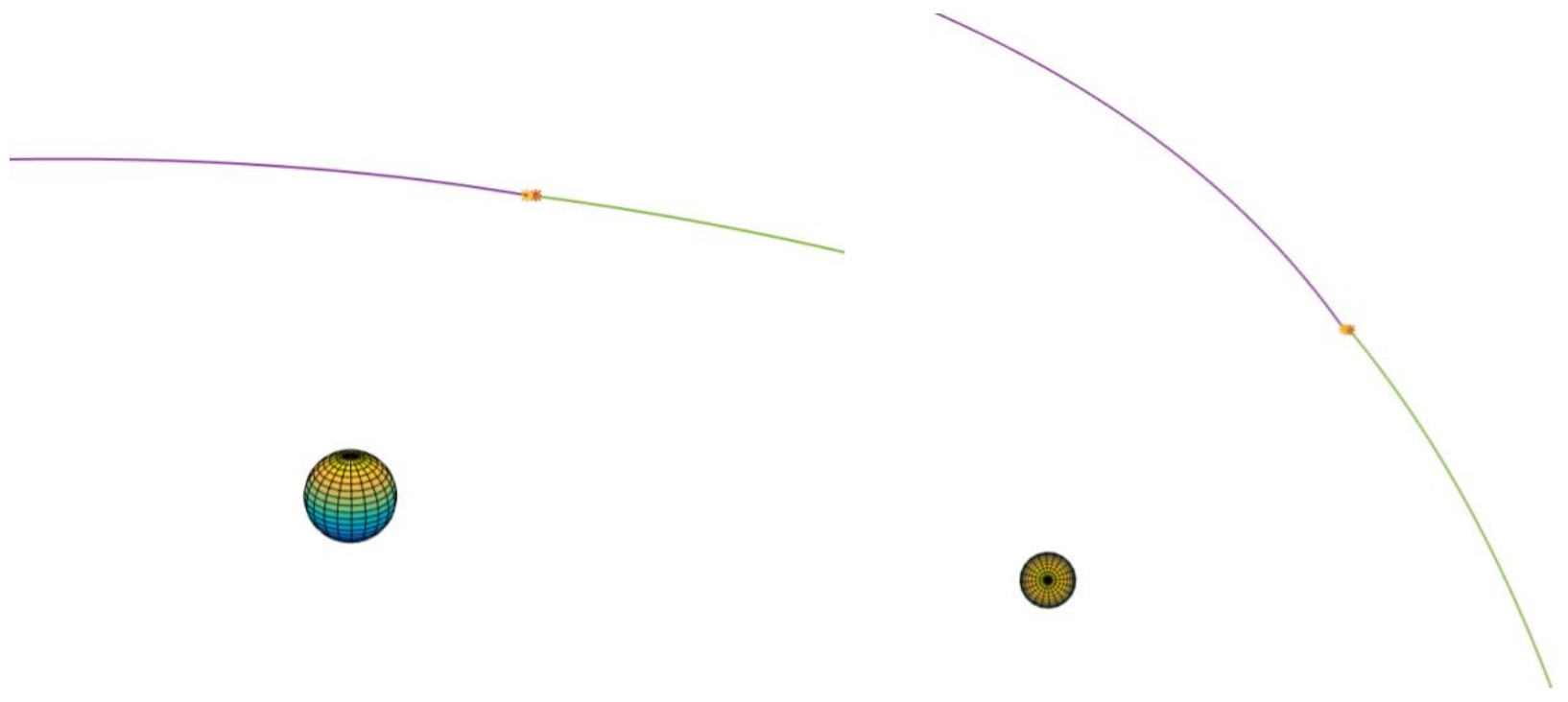

Figure 5.10: Close up views of transfer from hyperbolic escape trajectory (green) to manifold (purple) upon arrival at Saturn. The yellow star shows the injection point on the manifold and the red star shows the departure point on the hyperbola.

Again, these are certainly high values for $\Delta v$, but as can be seen in Table 5.5 they are significantly lower than the $\Delta v$ required for the traditional patched conics transfer approach that would escape Earth from a parking orbit and enter into a circular orbit about Saturn. The maneuver onto the hyperbolic escape trajectory to leave Earth is reduced by $4.07 \mathrm{~km} / \mathrm{s}$, while the arrival burn at Saturn is reduced by $2.70 \mathrm{~km} / \mathrm{s}$, bringing the total $\Delta v$ savings to $6.77 \mathrm{~km} / \mathrm{s}$ for the interplanetary transfer.

Table 5.5: Comparison of the $\Delta v$ required for traditional patched conics maneuvers and for the manifold maneuvers.

\begin{tabular}{c|ccc} 
Transfer Type & Departure $\Delta v$ & Arrival $\Delta v$ & Total $\Delta v$ \\
\hline Manifold & $5.74 \mathrm{~km} / \mathrm{s}$ & $1.76 \mathrm{~km} / \mathrm{s}$ & $7.49 \mathrm{~km} / \mathrm{s}$ \\
Traditional & $9.81 \mathrm{~km} / \mathrm{s}$ & $4.46 \mathrm{~km} / \mathrm{s}$ & $14.27 \mathrm{~km} / \mathrm{s}$
\end{tabular}

The $\Delta v$ to get to the halo orbit at Earth must also be included in the overall mission $\Delta v$, and the full $\Delta v$ budget with the total time of flight (ToF) is shown in Table 5.6. Even with the required transfer onto the halo orbit, the total $\Delta v$ is 
significantly less for the manifolds method. Although the time of flight is increased, the added time is comparable to missions that require multiply flybys.

Table 5.6: Comparison of the total $\Delta v$ required for mission and the time of flight (ToF) for traditional and manifold methods.

\begin{tabular}{c|cccc} 
Transfer Type & Halo Insertion $\Delta v$ & Transfer $\Delta v$ & Total $\Delta v$ & ToF \\
\hline Manifold & $3.19 \mathrm{~km} / \mathrm{s}$ & $7.49 \mathrm{~km} / \mathrm{s}$ & $10.68 \mathrm{~km} / \mathrm{s}$ & 2578 days \\
Traditional & $0 \mathrm{~km} / \mathrm{s}$ & $14.27 \mathrm{~km} / \mathrm{s}$ & $14.27 \mathrm{~km} / \mathrm{s}$ & 2219 days
\end{tabular}


Chapter 6

CONCLUSION

\subsection{Conclusions}

This thesis delved into the possibility of using the dynamics of the Circular Restricted Three-Body Problem to reduce $\Delta v$ required for interplanetary missions. A method of using the invariant manifolds of halo orbits to complete transfers to and from hyperbolic escape trajectories was used to test missions to both Jupiter and Saturn.

The results from this research have shown that using manifolds to aid in interplanetary transfers can significantly reduce $\Delta v$ for the burns required to enter and exit hyperbolic escape trajectories. For the Jupiter case, Earth escape $\Delta v$ using a manifold was $2.65 \mathrm{~km} / \mathrm{s}$ less than a transfer from a parking orbit, and at Jupiter the $\Delta v$ for capture was decreased $1.48 \mathrm{~km} / \mathrm{s}$. Although, the required $\Delta v$ to get to a halo orbit does mean that overall mission $\Delta v$ was only reduced by $1.04 \mathrm{~km} / \mathrm{s}$, this still shows that the CRTBP can reduce the energy required for transfers to Jupiter.

The outcome of the tests for a trajectory to Saturn yielded even better results than the Jupiter transfer. Even with the required $\Delta v$ to get the halo orbit the mission $\Delta v$ for the Saturn case was reduced by $3.6 \mathrm{~km} / \mathrm{s}$ from a traditional patched conics maneuver. This large reduction in $\Delta v$ has the potential to make a transfer to Saturn possible without the need for multiple flybys. The $\Delta v$ for the maneuver onto the hyperbolic escape trajectory at Earth was decreased by $4.1 \mathrm{~km} / \mathrm{s}$ and the arrival at Saturn was reduced by $2.7 \mathrm{~km} / \mathrm{s}$.

With further research and added optimization, this method for interplanetary transfers could reduce mission $\Delta v$ even further. 


\subsection{Future Work}

This thesis dedicated large amounts of time developing the tools necessary to model the dynamics of halo orbits and manifolds, as well as figure out methods to patch the different the sections of trajectories together for the maneuvers. With added optimization and further tests for different transfer cases, this method of completing interplanetary transfers could yield lower $\Delta v$ values, and could possibly be a promising approach to designing lower energy interplanetary transfer trajectories.

Using the Matlab tools that were developed for this thesis to calculate the dynamics of halo orbits, manifolds, and transfers to and from planetary orbits could be incorporated into an optimization scheme to find even farther reduced $\Delta v$ values for interplanetary transfers. The method of using manifolds to aid in transfers onto and off of hyperbolic escape trajectories could be further investigated, or more work could be done to accomplish transfers through using patched conics between the manifolds of departure and arrival planets. In addition, further research could be conducted to test itineraries that visit multiple planets through the use of manifolds.

Extensions of missions through use of the CRTBP to Jupiter and Saturn could also be investigated based off of the work from this thesis. While this research only studied the transfers from Earth to Jupiter and Saturn, more work could be done

on using manifolds to travel to interesting scientific locations within the systems of these planets. Using the arrival halo orbit as a base location spacecraft could then use invariant manifolds to travel to the planet itself or to one or multiple of the many moons surrounding these gas giants. This could be especially advantageous at Jupiter since the halo orbit would keep the spacecraft out of the harsh radiation environment surrounding the planet. 


\section{BIBLIOGRAPHY}

[1] E. Christian and A. Davis. Advanced Composition Explorer (ACE) Mission Overview. http://www.srl.caltech.edu/ACE/ace_mission.html.

[2] H. D. Curtis. Orbital Mechanics for Engineering Students, 3rd Edition. Butterworth-Heinemann, 2014.

[3] European Space Agency. SOHO Overview. https://www.esa.int/Our_Activities/Space_Science/SOHO_overview2.

[4] K. C. Howell. Three-dimensional, periodic, 'halo' orbits. Technical report, Stanford University, 1982.

[5] K. C. Howell, D. L. Mains, and B. T. Barden. Transfer trajectories from earth parking orbits to sun-earth halo orbits. In AAS/AIAA Spaceflight Mechanics Meeting, 1994.

[6] K. C. Howell and H. Pernicka. Numerical determination of lissajous trajectories in the restricted three-body problem. Technical report, Purdue University, 1986.

[7] W. S. Koon, M. W. Lo, J. E. Marsden, and S. D. Ross. Dynamical Systems, the Three-Body Problem and Space Mission Design. Cal Tech, 2011.

[8] M. Nakamiya, H. Yamakawa, D. J. Scheeres, and M. Yoshikawa. Interplanetary transfers between halo orbits: Connectivity between escape and capture trajectories. Journal of Guidance, Control, and Dynamics, 33(3), 2010.

[9] NASA. Interplanetary Superhighway Makes Space Travel Simpler. https://www.nasa.gov/mission_pages/genesis/media/jpl-release-071702.html. 
[10] NASA. ISEE. https://science.nasa.gov/missions/isee.

[11] NASA. James Webb Space Telescope. https://jwst.nasa.gov/about.html.

[12] NASA. Mission History.

https://genesismission.jpl.nasa.gov/gm2/mission/history.htm.

[13] NASA. WIND Spacecraft. https://wind.nasa.gov/.

[14] NASA. WMAP Mission Overview. https://map.gsfc.nasa.gov/mission/.

[15] S. D. Ross. Cylindrical Manifolds and Tube Dynamics in the Restricted Three-Body Problem. PhD thesis, California Institute of Technology, 2004.

[16] F. Topputo, M. Vasile, and F. Bernelli-Zazzera. Low energy interplanetary transfers exploiting invariant manifolds of the restricted three-body problem. Technical report, Politecnico di Milano, 2004.

[17] D. A. Vallado. Fundamentals of Astrodynamics and Applications, 4th Edition. Space Technology Library, 2013. 


\section{APPENDICES}

\section{Appendix A \\ ORBITAL ELEMENTS CALCULATION}

The following steps are used to calculate the orbital elements based on a position and velocity vector [2].

1. Calculate the specific angular momentum vector.

$$
\vec{h}=\vec{r}_{m} \times \vec{v}_{m}
$$

2. Find the inclination.

$$
i=\cos ^{-1}\left(\frac{h_{z}}{h}\right)
$$

3. Compute the vector defining the node line.

$$
\vec{N}=\left[\begin{array}{llll}
0 & 0 & 1
\end{array}\right] \times \vec{h}
$$

4. Find the value for RAAN.

$$
\begin{array}{r}
\Omega=\cos ^{-1}\left(\frac{N_{x}}{N}\right) \quad\left(N_{y} \geq 0\right) \\
\text { or } \\
\Omega=360^{\circ}-\cos ^{-1}\left(\frac{N_{x}}{N}\right) \quad\left(N_{y}<0\right)
\end{array}
$$

5. Calculate the eccentricity vector. Here, $\mu$ is the gravitational parameter of the Earth.

$$
\vec{e}=\frac{1}{\mu}\left(\vec{v}_{m} \times \vec{h}-\mu \frac{\vec{r}_{m}}{r_{m}}\right)
$$


6. Find the argument of perigee.

$$
\begin{array}{r}
\omega=\cos ^{-1}\left(\frac{\vec{N}}{N} \cdot \frac{\vec{e}}{e}\right) \quad\left(e_{z} \geq 0\right) \\
\text { or } \\
\omega=360^{\circ}-\cos ^{-1}\left(\frac{\vec{N}}{N} \cdot \frac{\vec{e}}{e}\right) \quad\left(e_{z}<0\right)
\end{array}
$$

7. Calculate the radial velocity.

$$
v_{r}=\frac{\vec{r}_{m} \cdot \vec{v}_{m}}{r_{m}}
$$

8. Find the true anomaly.

$$
\begin{array}{r}
\theta=\cos ^{-1}\left(\frac{\vec{e}}{e} \cdot \frac{r_{m}}{r_{m}}\right) \quad\left(v_{r} \geq 0\right) \\
\text { or } \\
\theta=360^{\circ}-\cos ^{-1}\left(\frac{\vec{e}}{e} \cdot \frac{\overrightarrow{r_{m}}}{r_{m}}\right) \quad\left(v_{r}<0\right)
\end{array}
$$


Appendix B

\section{VELOCITY VECTOR CALCULATION}

The following equation is used to obtain the velocity vector in the Earth-centered synodic frame [2].

$$
\vec{v}_{\text {park }}=Q_{p, s}\left(\frac{\mu}{h}\left[\begin{array}{llll}
-\sin (\theta) & e+\cos (\theta) & 0 & ]^{T}
\end{array}\right)\right.
$$

where $Q_{p, s}$ is the rotation matrix to transform a vector from the perifocal frame to the translated synodic frame.

$Q_{p, s}=\left[\begin{array}{ccc}-\sin \Omega \cos i \sin \omega+\cos \Omega \cos \omega & \cos \Omega \cos i \sin \omega+\sin \Omega \cos \omega & \sin i \sin \omega \\ -\sin \Omega \cos i \cos \omega-\cos \Omega \sin \omega & \cos \Omega \cos i \cos \omega-\sin \Omega \sin \omega & \sin i \cos \omega \\ \sin \Omega \sin i & -\cos \Omega \sin i & \end{array}\right]$ 